This is an Open Access article, distributed under the terms of the Creative Commons Attribution licence (http://creativecommons.org/licenses/by/4.0/), which permits unrestricted re-use, distribution, and reproduction in any medium, provided the original work is properly cited

doi:10.1017/S0956792516000450

\title{
Stable spike clusters for the one-dimensional Gierer-Meinhardt system
}

\author{
JUNCHENG WEI ${ }^{1}$ and MATTHIAS WINTER ${ }^{2}$ \\ ${ }^{1}$ Department of Mathematics, University of British Columbia, Vancouver, V6T 1Z2, B.C., Canada \\ email:jcwei@math.ubc.ca \\ ${ }^{2}$ Department of Mathematics, Brunel University London, Uxbridge UB8 3PH, UK \\ email:matthias.winter@brunel.ac.uk
}

(Received 14 May 2016; revised 08 October 2016; accepted 13 October 2016;

first published online 8 November 2016)

We consider the Gierer-Meinhardt system with precursor inhomogeneity and two small diffusivities in an interval

$$
\left\{\begin{array}{cc}
A_{t}=\varepsilon^{2} A^{\prime \prime}-\mu(x) A+\frac{A^{2}}{H}, & x \in(-1,1), t>0, \\
\tau H_{t}=D H^{\prime \prime}-H+A^{2}, & x \in(-1,1), t>0, \\
A^{\prime}(-1)=A^{\prime}(1)=H^{\prime}(-1)=H^{\prime}(1)=0, & \\
\text { where } 0<\varepsilon \ll \sqrt{D} \ll 1, & \\
\tau \geqslant 0 \text { and } \tau \text { is independent of } \varepsilon .
\end{array}\right.
$$

A spike cluster is the combination of several spikes which all approach the same point in the singular limit. We rigorously prove the existence of a steady-state spike cluster consisting of $N$ spikes near a non-degenerate local minimum point $t^{0}$ of the smooth positive inhomogeneity $\mu(x)$, i.e. we assume that $\mu^{\prime}\left(t^{0}\right)=0, \mu^{\prime \prime}\left(t^{0}\right)>0$ and we have $\mu\left(t^{0}\right)>0$. Here, $N$ is an arbitrary positive integer. Further, we show that this solution is linearly stable. We explicitly compute all eigenvalues, both large (of order $O(1)$ ) and small (of order $o(1)$ ). The main features of studying the Gierer-Meinhardt system in this setting are as follows: (i) it is biologically relevant since it models a hierarchical process (pattern formation of small-scale structures induced by a pre-existing large-scale inhomogeneity); (ii) it contains three different spatial scales two of which are small: the $O(1)$ scale of the precursor inhomogeneity $\mu(x)$, the $O(\sqrt{D})$ scale of the inhibitor diffusivity and the $O(\varepsilon)$ scale of the activator diffusivity; (iii) the expressions can be made explicit and often have a particularly simple form.

Key words: $35 \mathrm{~B} 35 ; 35 \mathrm{~J} 75 ; 35 \mathrm{~K} 57 ; 35 \mathrm{~K} 58 ; 92 \mathrm{C} 15$

\section{Introduction}

In his pioneering work [31] in 1952, Turing studied how pattern formation could start from an unpatterned state. He explained the onset of pattern formation by the presence 
of spatially varying instabilities combined with the absence of spatially homogeneous instabilities. This approach is now commonly called Turing diffusion-driven instability. Since then many reaction-diffusion systems in biological modelling have been proposed and the occurrence of pattern formation has been investigated based on the approach of Turing instability [31]. One of the most widely used class of biological patternformation models consists of the activator-inhibitor type models which are based on real-world interactions such as those encountered in experiments and observations on seashells, animal skin patterns, embryological development, cell signalling pathways and many more. Amongst these, one of the most popular models is the Gierer-Meinhardt system $[11,16,18]$, which in one dimension with a precursor-inhomogeneity and two small diffusivities can be stated as follows:

$$
\begin{cases}A_{t}=\varepsilon^{2} \Delta A-\mu(x) A+\frac{A^{2}}{H}, & x \in(-1,1), t>0, \\ \tau H_{t}=D \Delta H-H+A^{2}, & x \in(-1,1), t>0, \\ A^{\prime}(-1)=A^{\prime}(1)=H^{\prime}(-1)=H^{\prime}(1)=0, & \end{cases}
$$

$$
\text { where } 0<\varepsilon \ll \sqrt{D} \ll 1 \text {, }
$$

$\tau \geqslant 0$ and $\tau$ is independent of $\varepsilon$.

In the standard Gierer-Meinhardt system without precursor, it is assumed that $\mu(x) \equiv 1$.

Precursor gradients in reaction-diffusion systems have been investigated in earlier work. The original Gierer-Meinhardt system [11,16,18] has been introduced with precursor gradients. These precursors were proposed to model the localization of the head structure in the coelenterate Hydra. Gradients have also been used in the Brusselator model to restrict pattern formation to some fraction of the spatial domain [14]. In that example, the gradient carries the system in and out of the pattern-forming part of the parameter range (across the Turing bifurcation), thus effectively confining the domain where peak formation can occur.

In this paper, we study the Gierer-Meinhardt system with precursor and prove the existence and stability of a cluster, which consists of $N$ spikes approaching the same limiting point.

More precisely, we prove the existence of a steady-state spike cluster consisting of $N$ spikes near a non-degenerate local minimum point $t^{0}$ of the positive inhomogeneity $\mu(x) \in C^{3}(\Omega)$, i.e. we assume that $\mu^{\prime}\left(t^{0}\right)=0, \mu^{\prime \prime}\left(t^{0}\right)>0$ and we have $\mu\left(t^{0}\right)>0$. Further, we show that this solution is linearly stable.

We explicitly compute all eigenvalues, both large (of order $O(1)$ ) and small (of order $o(1)$ ). The main features of studying the Gierer-Meinhardt system in this setting are as follows:

(i) It is biologically relevant since it models a hierarchical process (pattern formation of small-scale structures induced by a pre-existing inhomogeneity).

(ii) It is important to note that this system contains three different spatial scales two of which are small (i.e. $o(1))$ : 
(a) The $O(1)$ scale of the precursor $\mu(x)$.

(b) The $O(\sqrt{D})$ scale of the inhibitor diffusivity.

(c) The $O(\varepsilon)$ scale of the activator diffusivity.

Consequently, there are the two small quantities $\sqrt{D}$ and $\frac{\varepsilon}{\sqrt{D}}$ which play an important role throughout the paper.

(iii) The expressions can be made explicit and often have a particularly simple form.

Let us now summarize the analytical approach employed in our paper. The existence proof is based on Liapunov-Schmidt reduction. The stability of the cluster is shown by first separating the eigenvalues into two cases: Large eigenvalues which tend to a non-zero limit and small eigenvalues which tend to zero in the limit $D \rightarrow 0$ and $\frac{\varepsilon}{\sqrt{D}} \rightarrow 0$. Large eigenvalues are then explored by deriving suitable non-local eigenvalue problems (NLEPs) based on a compactness argument of Dancer [6]. Small eigenvalues are calculated explicitly by using asymptotic analysis with rigorous error estimates.

We shall establish the existence and stability of positive $N$-peaked steady-state spike clusters to (gm1). The steady-state problem for positive solutions of (1.1) is the following:

$$
\begin{cases}\varepsilon^{2} A^{\prime \prime}-\mu(x) A+\frac{A^{2}}{H}=0, & x \in(-1,1), \\ D H^{\prime \prime}-H+A^{2}=0, & x \in(-1,1), \\ A(x)>0, H(x)>0, & x \in(-1,1), \\ A^{\prime}(-1)=A^{\prime}(1)=H^{\prime}(-1)=H^{\prime}(1)=0 . & \end{cases}
$$

To have a non-trivial spike cluster, we assume throughout the paper that

$$
N \geqslant 2
$$

Before stating our main results, let us review some previous results on pattern formation for the Gierer-Meinhardt system (1.1), in particular concerning spiky patterns.

(1) I. Takagi [30] proved the existence of $N$-spike steady-state solutions of (1.1) in an interval for homogeneous coefficients (i.e. $\mu(x)=1$ ) in the regime $\varepsilon \ll 1$ and $D \gg 1$, where $N$ is an arbitrary positive integer. For these solutions, the spikes are identical copies of each other and their maxima are located at

$$
t_{j}=-1+\frac{2 j-1}{N}, \quad j=1, \ldots, N
$$

The proof in [30] is based on symmetry and the implicit function theorem.

(2) In [15] (using matched asymptotic expansions) and [43] (based on rigorous proofs), the following stability result has been shown: For the $N$-spike steady-state solution derived in item 1 and $0 \leqslant \tau<\tau_{0}(N)$, where $\tau_{0}(N)>0$ is independent of $\varepsilon$, there are numbers $D_{1}>D_{2}>\cdots>D_{N}>\cdots$ (which have been computed explicitly) such that the $N$-spike steady state is stable for for $D<D_{N}$ and unstable for $D>D_{N}$. 
(3) In [15] (using matched asymptotic expansions) and [33] (based on rigorous analysis), the following existence and stability results have been shown: For a certain parameter range of $D$, the Gierer-Meinhardt system $(1.1)$ with $\mu(x)=1$ has asymmetric $N$-spike steady-state solutions, which consist of exact copies of precisely two different spikes with distinct amplitudes. They can be considered as bifurcating solutions from those in item 1 such that the amplitudes start to differ at the bifurcation point (saddle-node bifuraction). The stability of these asymmetric $N$-peaked solutions has been studied in [33].

(4) In [45], the existence and stability of $N$-peaked steady states for the Gierer-Meinhardt system with precursor inhomogeneity has been shown. These spikes have different amplitudes. In particular, the results imply that precursor inhomogeneities can induce instabilities. Single-spike solutions for the Gierer-Meinhardt system with precursor including spike motion have been studied in [32].

(5) In [42], the existence of symmetric and asymmetric multiple spike clusters in an interval has been shown.

Compared to each of the items listed above, the setting and results in our paper have marked differences. We now consider two small parameters, $D$ and $\frac{\varepsilon}{\sqrt{D}}$ which results in new types of behaviour. The leading-order asymptotic expression of the large and small eigenvalues depend on the index of the eigenvalue quadratically, whereas in items 1 and 2, this relation is oscillatory (involving trigonometric functions).

In our study, the spikes in leading order have equal amplitudes and uniform spacing, although there is precursor inhomogeneity in the system, in contrast to item 3 . The amplitudes, positions and eigenvalues in our study can be characterized explicitly and have a simpler form than in item 4 . We can also prove the stability of clusters not merely their existence as in item 5. In particular, we show here that the clusters may be stable, whereas in item 5 they are expected to be unstable.

In the shadow system case $(D=\infty)$, the existence of single- or $N$-peaked solutions has been established in $[12,13,21,22]$ and other papers. It is interesting to remark that symmetric and asymmetric patterns can also be obtained for the Gierer-Meinhardt system on the real line, see [7,8]. We refer to [23] for the singular limit eigenvalue problem (SLEP) approch for the existence and stability of multi-layered solutions for reaction-diffusion systems. For two-dimensional domains, the existence and stability of multi-peaked steady states has been proved in [38-40] and results similar to items 1 and 2 have been derived. Hopf bifurcation has been established in $[6,34,35,40]$. The repulsive dynamics of multiple spikes has been studied in [9].

Another study with three different spatial scales, two of which are small, considers a consumer chain model allowing for a novel type of spiky clustered pattern which is stable for certain parameters [46].

The model in our paper shows some similarity to variational models for material microstructure $[1,20,48]$. In both models, the solutions have two small scales. However, in our case, we have two parameters to control each of them independently, whereas in the microstructure case they are expressions of different orders depending on the same small parameter and so they are related to one another. 
Results on the existence and stability of multi-spike steady states have been reviewed and put in a general context in [47].

We plan to continue the investigation of stable clusters in future research. In particular, we are interested in two-dimensional patterns. Whereas in one space dimension the spikes in the cluster are aligned, in two dimensions we expect a rather rich geometric picture of possible spike locations.

This paper has the following structure: In Section 2, we state our main results on existence and stability. Then, we show some numerical simulations to illustrate the main results. We also study the dynamics of pattern formation, even outside the regime covered by the results. Next, we present four highlights of the results and their proofs. Finally, we sketch the main steps of the proofs. In Section 3, we introduce some preliminaries. In Sections 4-7, we prove the existence of steady-state spike clusters: In Section 4, we introduce suitable approximate solutions, in Section 5, we compute their error, in Section 6, we use the Liapunov-Schmidt method to reduce the existence of solutions of (1.2) to a finite-dimensional problem, in Section 7, we solve this finite-dimensional reduced problem. In Sections 8, 9 and Appendix B, we prove the stability of these steady-state spike clusters: In Section 8, we study the large eigenvalues of the linearized operator and show that it has diagonal form. We give a complete description of their asymptotic behaviour which is stated in Lemma 15. In Section 9, we characterize the small eigenvalues of the linearized operator and show that they all have negative real part. This includes deriving the eigenvalues of a matrix which is needed to compute the small eigenvalues explicitly. We give a complete description of their asymptotic behaviour in leading order which can be found in Lemma 16. Our approach here is to interpret the main matrix as the finitedifference approximation of a suitable ordinary differential equation, compute the solution of this approximation explicitly and get the eigenvectors by taking its values at uniformly spaced points. In Section 10, we conclude with a discussion of our results with respect to the bridging of length scales and the hierarchy of multi-stage biological processes. In Appendix A, we state a few results on NLEPs which are needed throughout the paper. In Appendix B, we perform the technical analysis needed to derive the small eigenvalues.

\section{Main results on existence and stability}

In this section, we state our main results on existence and stability of solutions and present four highlights of our approach and sketch the proofs of the main results.

We first need to introduce some essential notation. Let $L^{2}(-1,1)$ and $H^{2}(-1,1)$ denote Lebesgue and Sobolev space, respectively. Let the function $w$ be the unique solution (ground state) of the problem

$$
\begin{cases}w^{\prime \prime}-w+w^{2}=0, & y \in \mathbb{R}, \\ w>0, & w(0)=\max _{y \in \mathbb{R}} w(y), \\ w(y) \rightarrow 0 & \text { as }|y| \rightarrow \infty .\end{cases}
$$

Then, $w(y)$ can explicitly be written as

$$
w(y)=\frac{3}{2} \cosh ^{-2}\left(\frac{y}{2}\right) .
$$


Elementary calculations give

$$
\int_{\mathbb{R}} w^{2}(z) d z=6, \quad \int_{\mathbb{R}} w^{3}(z) d z=7.2, \quad \int_{\mathbb{R}}\left(w^{\prime}\right)^{2}(z) d z=1.2 .
$$

Let

$$
\Omega=(-1,1)
$$

Let $t^{0} \in(-1,1)$ and set

$$
\mu^{0}=\mu\left(t^{0}\right)
$$

Let $\hat{\xi}^{0}$ satisfy

$$
\hat{\xi}^{0} \sim \frac{1}{\left(\mu^{0}\right)^{3 / 2}}
$$

compare (4.30).

We set

$$
\xi_{\varepsilon}:=\frac{2 \sqrt{D}}{\varepsilon \int_{\mathbb{R}} w^{2}(z) d z} .
$$

Our first result is about the existence of an $N$-spike cluster solution near a non-degenerate local minimum point of the precursor.

Theorem 1 (Existence of an $N$-spike cluster) Let $N$ be a positive integer and $t^{0} \in(-1,1)$. We assume that $\mu \in C^{3}(-1,1)$ and

$$
\mu^{\prime}\left(t^{0}\right)=0, \mu^{\prime \prime}\left(t^{0}\right)>0
$$

Then, for $\varepsilon \ll \sqrt{D} \ll 1$, problem (1.2) has an $N$-spike cluster solution which concentrates at $t^{0}$. In particular, it satisfies

$$
\begin{gathered}
A_{\varepsilon}(x) \sim \sum_{k=1}^{N} \xi_{\varepsilon} \hat{\xi}^{0} \mu^{0} w\left(\sqrt{\mu^{0}} \frac{x-t_{k}^{\varepsilon}}{\varepsilon}\right), \\
H_{\varepsilon}\left(t_{k}^{\varepsilon}\right) \sim \xi_{\varepsilon} \hat{\xi}^{0}, \quad k=1, \ldots, N, \\
t_{k}^{\varepsilon} \rightarrow t^{0}, \quad k=1, \ldots, N,
\end{gathered}
$$

where $\mu^{0}$ has been defined in (2.4), $\hat{\xi}^{0}$ has been introduced in (2.5) and $\xi_{\varepsilon}$ has been defined in (2.6).

Next, we state our second result which concerns the stability of the $N$-spike cluster steady states given in Theorem 1 .

Theorem 2 (Stability of an $N$-spike cluster) For $\varepsilon \ll \sqrt{D} \ll 1$, let $\left(A_{\varepsilon}, H_{\varepsilon}\right)$ be an $N$-spike cluster steady state given in Theorem 1. Then, there exists $\tau_{0}>0$ independent of $\varepsilon$ and $\sqrt{D}$ such that the $N$-spike cluster steady state $\left(A_{\varepsilon}, H_{\varepsilon}\right)$ is linearly stable for all $0 \leqslant \tau<\tau_{0}$. 

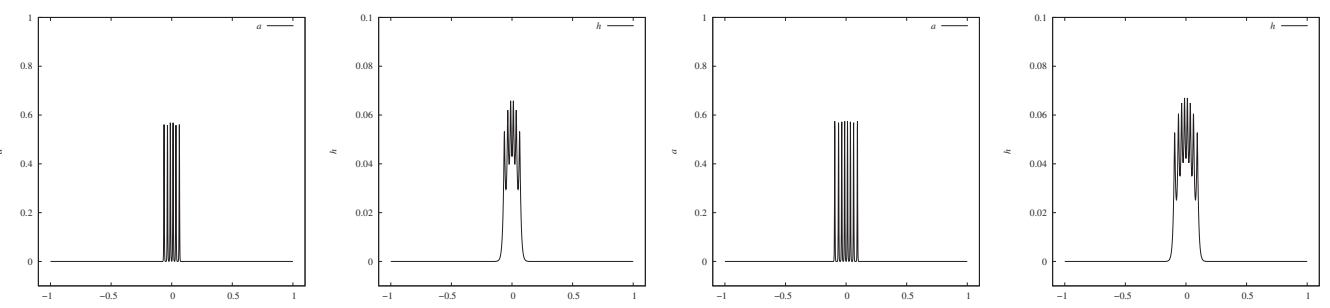

FIGURE 1. Clustered spiky steady states of (1.1) for $\varepsilon^{2}=0.00001, D=0.001, \mu(x)=6+$ $400 x^{2}$ and $\mu(x)=6+200 x^{2}$, respectively. Shown are a six-spike cluster and an eight-spike cluster. In both cases, the activator $a$ is displayed in the left graph and the inhibitor $h$ in the right graph.

Now by performing some numerical simulations, we study patterns for the GiererMeinhardt system with precursor gradient given in (1.1) systematically in various situations.

Throughout all these simulations, we choose $\tau=0.1, \varepsilon^{2}=0.00001$ and take varying values for $D$ ranging between a few times $\varepsilon^{2}$ and 1 .

Further, we vary the strength of the precursor gradient and observe two distinct types of behaviour: For strong precursor gradient, the spikes assemble as a cluster near the global minimum point of the precursor, for weak precursor gradient, the spikes are distributed over the whole interval.

We will observe a rather rich dynamical behaviour which by far exceeds the immediate vicinity of the spike cluster which will be analysed in detail in this paper.

First, we show the results of computations of spike cluster steady states of (1.1). These have been obtained as long-time limits of (1.1) and are numerically stable.

In Figure 1, we note that the amplitudes of the different spikes of a cluster in the activator component are very close to each other.

On the other hand, the inhibitor values differ substantially at different spike locations and are highest near the centre of the cluster. This stands in contrast to the precursor $\mu(x)$ which attains its global minimum at the centre of the cluster. The combination of these two effects leads to almost equal spike amplitudes.

Both the activator and inhibitor peaks have almost equal distance.

Now, we show the initial condition which has been used in all simulations of clusters and multiple spikes.

Next, we display the dynamics of getting a cluster from the initial condition given in Figure 2.

In Figure 3, for $t=0$, we start with very fine oscillations of the activator $a$. Then, for $t=0.70$, we reach a pattern which is very close to zero except in the centre of the interval where fine oscillations still prevail. Starting from $t=0.80$, we see eight spikes which generally increase in amplitude and whose positions are mainly fixed. Looking more closely, we can also see that the amplitudes show some oscillatory behaviour, first overshooting the final amplitude of around 0.57 and then oscillating around the final amplitude and approaching it.

Next, we consider a different regime of a weaker precursor gradient (all other parameters remain unchanged from before). We observe spikes which are distributed over the whole interval. 

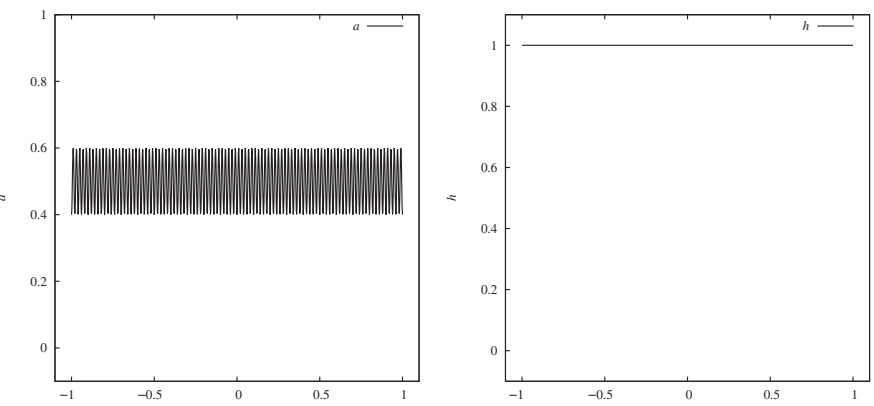

FIGURE 2. Initial condition of cluster and multiple spikes, activator and inhibitor are shown. We choose a large number of oscillations for the activator in order to get as many spikes as possible.
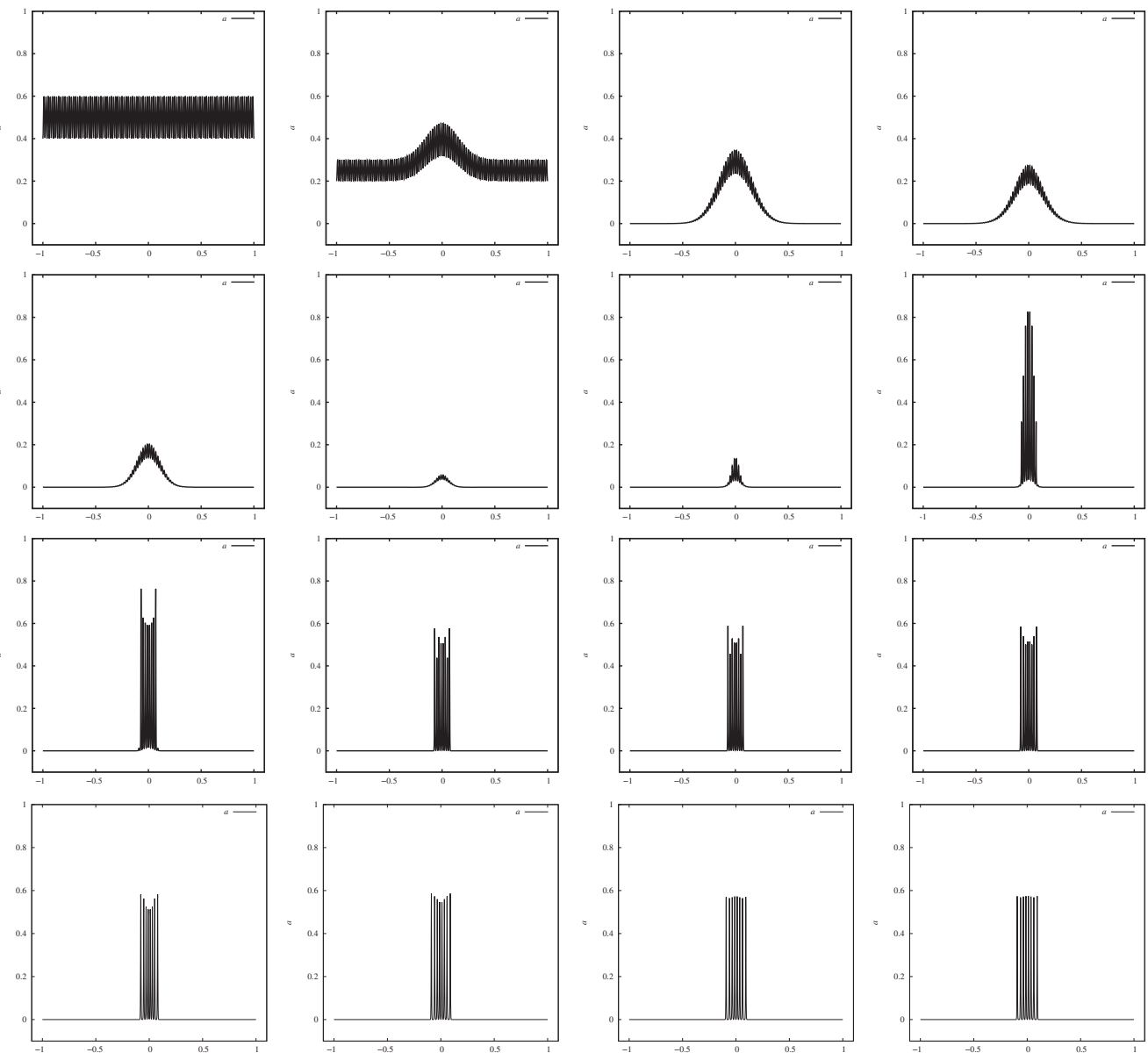

Figure 3. Dynamics of a spike cluster for $t=0,0.05,0.10,0.20,0.30,0.50,0.70,0.80,1.0,5.0$, $10,50,100,500,1,000,5,000$ and $D=0.001$. The activator is shown. The initial oscillations are transformed into a spike cluster located at the global minimum point of the precursor inhomogeneity $\mu(x)=6+200 x^{2}$. 

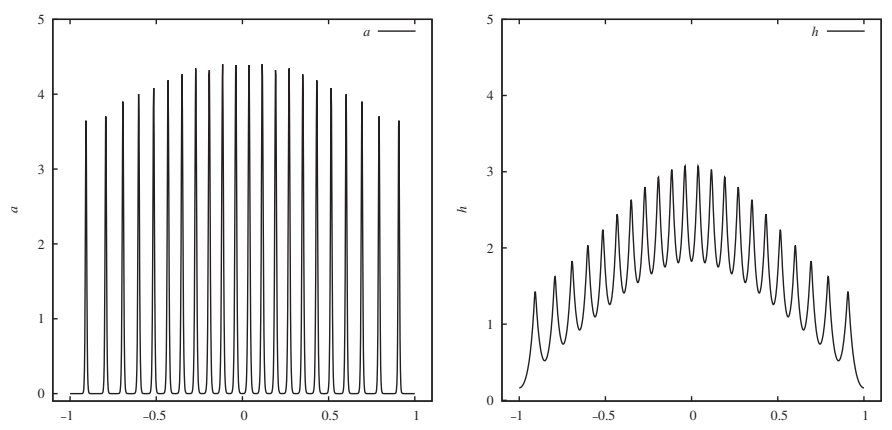

FIGURE 4. Multi-spike pattern consisting of 22 spikes distributed over the whole domain for $D=0.001$ and precursor inhomogeneity $\mu(x)=1+x^{2}$. Activator and inhibitor are shown.

Now, we display the dynamics of getting a multiple spike pattern from the initial condition given in Figure 2.

In Figure 5, we start with very fine oscillations of the activator $a$. Immediately, the amplitudes of the oscillations change driven by the precursor gradient, but their period remains mainly unchanged. Then, spikes start to form, first at the boundary, then further and further inside the interval. Finally, the spikes in the centre of the domain form almost simultaneously and there is also some oscillatory behaviour between their amplitudes. It takes longest for the amplitudes of the two spikes in the centre to increase to their steady-state value. The positions of the spikes are mainly unchanged but the increase in amplitude for the two central spikes is coupled with them slowly moving apart and pushing the remaining spikes away from the centre.

In Figure 6, we show how the number of multiple spikes depends on the diffusion constants.

Finally, in Figure 7, we show the behaviour as the two small diffusion constants $\varepsilon^{2}$ and $D$ come close to each other.

Remark 3 For the stability, we assume that $0 \leqslant \tau<\tau_{0}$ for some $\tau_{0}>0$. Stability in the case where $\tau$ is large has been investigated in [35] for single spikes and those results on Hopf bifurcation are expected to carry over to the case of an $N$-spike cluster considered here. We remark that stability in the case of large $\tau$ for the shadow system has been studied in [6,34]. It turns out that this Hopf bifurcation leads to oscillations of the amplitudes. The Hopf bifurcation at $\tau=\tau_{0}$, where $\tau_{0}$ is of order 1 , still arises even in the regime $0 \ll \varepsilon \ll \sqrt{D} \ll 1$ considered in this paper. For the spike cluster, $\tau_{0}$ is independent of $N$ which can be shown by the analysis in Sections 8 and 9.

Remark 4 It is an interesting question to consider the maximum number of stable spikes in the regime $0 \ll \varepsilon \ll \sqrt{D} \ll 1$ studied in this paper. We expect that there are stable multispike solutions if $N<\frac{c}{\sqrt{D}}$ (in leading order of $D$ ). In the regime $N \sim \frac{c}{\sqrt{D}}$, the spikes will be distributed over the whole interval and at $N \sim \frac{c}{\sqrt{D}}$, we expect an overcrowding instability. This threshold would be an extension of the corresponding result for $D=1$ (see [43]). The cluster solutions studied in this paper are only possible if $N<\frac{c}{\sqrt{D} \log \frac{1}{D}}$ (in leading order of $D)$ due to the distance between spikes. We have presented some numerical simulations of 

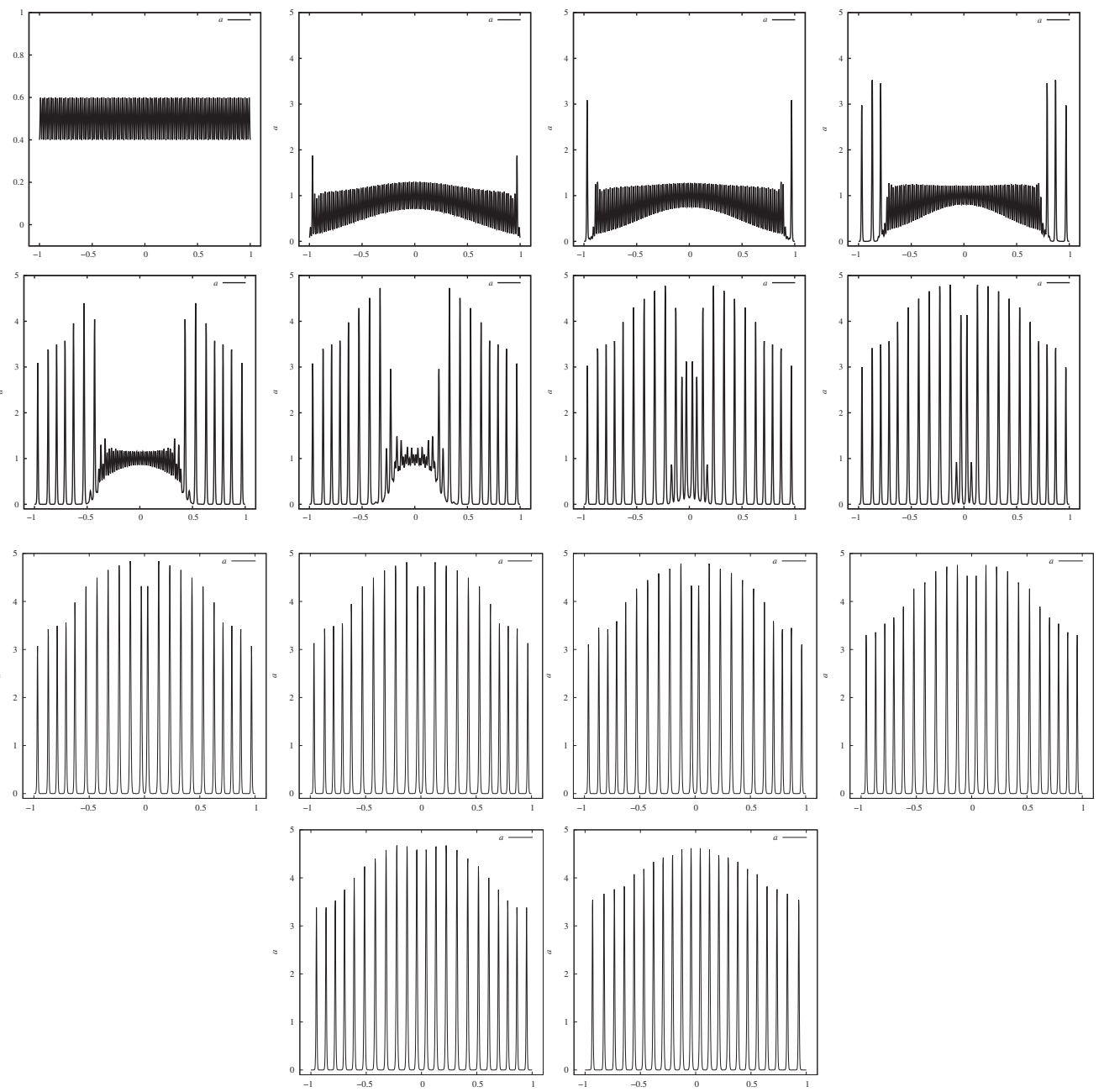

FIGURE 5. Dynamics of multiple spikes distributed over whole domain for $t=0,3,5,10,20,25$, $30,35,40,50,100,500,1,000,5,000$. Further, we have $D=0.001$ and precursor inhomogeneity $\mu(x)=1+x^{2}$. The activator is shown.

both situations: A spike cluster as well as multiple spikes distributed over the whole domain. (For the cluster, see Figure 1, for multiple spikes see Figures 4 and 6. Their dynamics are shown in Figures 3 and 5, respectively.)

Remark 5 If $\frac{\varepsilon}{\sqrt{D}} \sim 1$, the spike solutions will change into other types of patterns, e.g. spatial oscillations, which could again be stable. It is also possible that the patterns will vanish. We have presented some numerical examples to illustrate this behaviour (see Figure 7).

Remark 6 Previous studies of the precursor case can be found amongst others in [2,27,28]. We also refer to results for the dynamics of pulses in heterogeneous media [24,49]. This clustered spike pattern and multiple spikes distributed over the whole interval are more regular than multiple spike patterns observed for the Gierer-Meinhardt system with precursor 

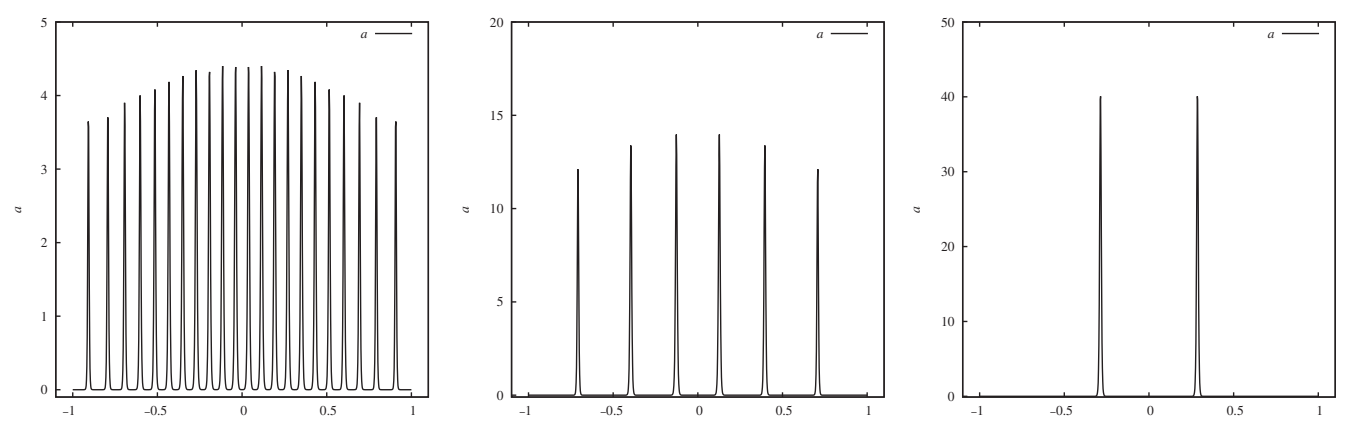

FIGURE 6. Multiple spikes are displayed for $D=0.001,0.01,0.1$ and precursor inhomogeneity $\mu(x)=1+x^{2}$. The activator is shown. We get 22, 6 and 2 spikes, respectively. The number of spikes decreases with increasing $D$ which can be understood by an overcrowding instability.
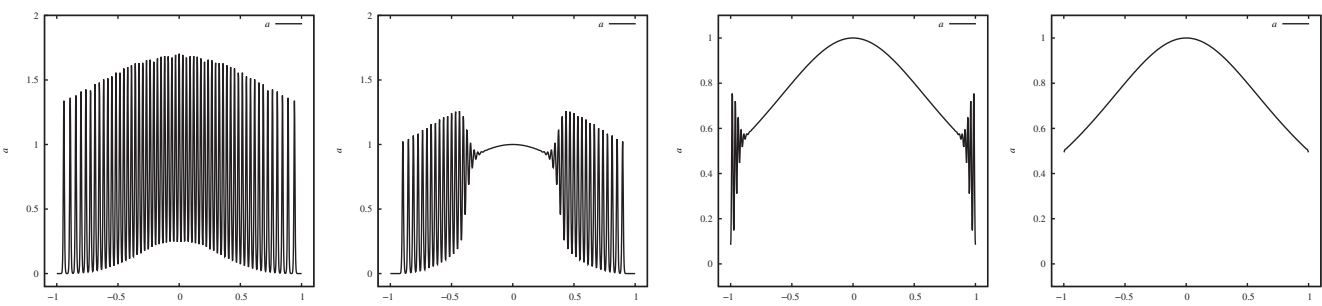

FIGURE 7. A combination of oscillations and pattern-free state is displayed for $D=0.0001,0.00005$, $0.00003,0.00002$. Here, $D$ approaches $\varepsilon^{2}=0.00001$. The activator is shown. For decreasing $D$, the oscillations disappear, starting in the centre of the domain, which can be explained by the limited range of Turing instability.

and order 1 inhibitor diffusivity studied in [45]. In that case, multiple spike patterns have irregular distances and amplitudes since the precursor interacts with the geometry of the domain (represented by the Green's function) globally. On the other hand, for the regime covered in this paper, the precursor acts globally but the Green's function acts only locally between neighbouring spikes.

The proofs of both Theorems 1 and 2 will follow the approach in [47], where we reviewed and discussed many results on the existence and stability of multi-spike steady states. Before providing a sketch of the proofs of Theorems 1 and 2, we first state four highlights of the results and proofs in an informal manner. For each of these, we also indicate the novelty in comparison to previous work.

Highlight 1: For the proof in Theorem 1, we use Liapunov-Schmidt reduction to derive a reduced problem which will determine the positions of the spikes. This reduced problem in leading order is given by

$$
W_{0}(\mathbf{t}) \sim c_{1} \sum_{k,|k-s|=1} e^{-\left|t_{s}-t_{k}\right| / \sqrt{D}}\left(-\frac{t_{s}-t_{k}}{\left|t_{s}-t_{k}\right|}\right)+c_{2} \sqrt{D} \mu^{\prime \prime}\left(t^{0}\right)\left(t_{s}-t^{0}\right), \quad s=1, \ldots, N
$$

where $c_{1}, c_{2}>0$ are constants which are independent of the small parameters and $\mathbf{t}=\left(t_{1}, \ldots, t_{N}\right)$ are the positions of the spikes (compare (7.4)). We need to solve $W_{0}(\mathbf{t})=0$, 
which implies

$$
t_{s}-t_{s-1} \sim \sqrt{D} \log \frac{1}{D}, \quad s=2, \ldots, N,
$$

(compare (7.6)). The distance between neighbouring spikes in the cluster is small (converging to zero) and in leading order, it is the same between any pair of neighbours. Further note that the distance is determined by the large diffusion constant $D$. In previous work for $D=O(1)$, the spikes either have distance of order 1 or, in the case of clusters, they have small distance which is determined by the small diffusion constant $\varepsilon$. Thus, the spacing between neighbouring spikes follows a new asymptotic rule not encountered before.

The deeper reason for this behaviour is that the spike cluster we consider in this paper is formed by balancing the interactions of the inhibitor between neighbouring spikes and the inhomogeneity. On the other hand, in [42] and other previous work on spike clusters, they are established by balancing the interactions of the activator between neighbouring spikes and the inhomogeneity.

Highlight 2: The large eigenvalues with $\lambda_{\varepsilon} \rightarrow \lambda_{0} \neq 0$ and their corresponding eigenfunctions

$$
\phi_{\varepsilon, i}(y) \rightarrow \phi_{i}(y), \quad y=\frac{x-t_{i}}{\varepsilon}, \quad i=1, \ldots, N,
$$

where $\phi_{\varepsilon, i}(y)$ is the restriction of the re-scaled eigenfunction of the activator $A_{\varepsilon}$ near $t_{i}$, in the limit $\max \left(\frac{\varepsilon}{\sqrt{D}}, D\right) \rightarrow 0$ solve the NLEP

$$
\Delta_{y} \phi_{i}-\phi_{i}+2 w \phi_{i}-\frac{2 \int_{\mathbb{R}} w \phi_{i} d y}{\int_{\mathbb{R}} w^{2} d y} w^{2}=\lambda_{0} \phi_{i}, \quad i=1, \ldots, N,
$$

(see (8.6)). This NLEP has diagonal form. Thus, with respect to large eigenvalues each spike only interacts with itself and not with the other spikes. It follows that the spike cluster is stable with the respect to large eigenvalues. In previous work for the case $D=O(1)$, the stability problem of large eigenvalues for multiple spikes leads to a vectorial NLEP. It has to be studied by considering the spectrum of the matrix involved. Depending on the parameters, the multiple spikes can be stable or unstable. In the case of clusters for $D=O(1)$, the stability has not been considered rigorously but we expect that the solution is unstable.

Highlight 3: The small eigenvalues $\lambda_{\varepsilon} \rightarrow 0$ in leading order are given by the eigenvalues of the matrix

$$
-\varepsilon^{2} c_{3} \mathcal{M}\left(\mathbf{t}^{0}\right)
$$

where $c_{3}>0$ is independent of the small parameters, $\mathbf{t}^{0}=\left(t^{0}, \ldots, t^{0}\right)$ and

$$
\begin{aligned}
& \mathcal{M}\left(\mathbf{t}^{0}\right)_{i, j=1}^{N} \sim \mu^{\prime \prime}\left(t^{0}\right)\left[\operatorname { l o g } \frac { 1 } { D } \left[-(i-1)(N+1-i) \delta_{i, j-1}-i(N-i) \delta_{i, j+1}\right.\right. \\
& \left.\left.\quad+[(i-1)(N+1-i)+i(N-i)] \delta_{i, j}\right]+4 \delta_{i, j}\right]
\end{aligned}
$$

with $\delta_{N, 0}=\delta_{1, N+1}=0$ (compare 9.13). The tridiagonal matrix $\mathcal{M}\left(\mathbf{t}^{0}\right)$ derived here indicates that with respect to small eigenvalues each spike only interacts with its nearest neighbour 
and not with the other spikes. This is different from the case $D=O(1)$ : For multiple spikes, the matrix typically has strictly positive entries only (although it could have zero eigenvalues in the presence of symmetries), for clusters, a similar tridiagonal matrix which depends on $\varepsilon$ has been studied to show the existence of spike clusters, see [42].

Highlight 4: We determine all the eigenvalues of the matrix $\mathcal{M}\left(\mathbf{t}^{0}\right)$ (see Highlight 3) explicitly by a method based on exactly finding a finite-difference approximation to a suitable ordinary differential equation.

These eigenvalues are given by

$$
\lambda_{n, \varepsilon} \sim-\varepsilon^{2} \log \frac{1}{D} c_{3} \mu^{\prime \prime}\left(t^{0}\right) n(n+1), \quad n=1, \ldots, N-1 .
$$

Further, there is an eigenvalue of smaller size given by

$$
\lambda_{0, \varepsilon} \sim-\varepsilon^{2} 4 c_{3} \mu^{\prime \prime}\left(t^{0}\right)
$$

(compare Lemma 16). This implies that the spike cluster is stable with respect to small eigenvalues. This result seems to be new in the literature. Therefore, we provided this proof.

Finally, we give a sketch of the proofs of Theorem 1 (existence) and Theorem 2 (stability).

We begin by stating the main steps in the proof of Theorem 1:

(1) The existence problem (4.20) is reduced to a non-local one-dimensional problem

$$
\mathcal{S}_{\varepsilon}[\hat{A}]=\varepsilon^{2} \hat{A}^{\prime \prime}-\mu(x) \hat{A}+\frac{\hat{A}^{2}}{T[\hat{A}]}=0,
$$

where

$$
T[\hat{A}]=\int_{\mathbb{R}} G_{D}(\cdot ; z) \hat{A}^{2}(z) d z
$$

is an integral operator solving (4.21).

(2) The ansatz for a spike cluster is

$$
\hat{A}(x)=\sum_{k=1}^{N} \hat{\xi}_{k} \tilde{w}_{k}(x)
$$

(see (2.8)) with $\hat{\xi}_{k}$ determined by the amplitude identity

$$
\hat{\xi}_{k}=T[\hat{A}]\left(t_{k}\right) \quad \text { for } k=1, \ldots, N .
$$

(3) The amplitude identity is crucial to show that $\mathcal{S}_{\varepsilon}[\hat{A}]$ is small in an appropriate norm. Therefore, $\hat{A}$ is intuitively almost a solution.

(4) Using the estimate on $\mathcal{S}_{\varepsilon}[\hat{A}]$, one can perform Liapunov-Schmidt reduction resulting in a small

$$
\phi_{\varepsilon} \perp \operatorname{span}\left\{\tilde{w}_{k}^{\prime}, k=1, \ldots, N\right\} \subset H^{2}(\Omega)
$$


such that

$$
\mathcal{S}_{\varepsilon}\left[\hat{A}+\phi_{\varepsilon}\right] \in \mathcal{C}=\operatorname{span}\left\{\tilde{w}_{k}^{\prime}, k=1, \ldots, N\right\} \subset L^{2}(\Omega) .
$$

(5) We find a solution close to $\hat{A}$ if $\mathcal{S}_{\varepsilon}\left[\hat{A}+\phi_{\varepsilon}\right] \perp \mathcal{C}$ is satisfied. Solving the finitedimensional problem $\mathcal{S}_{\varepsilon}\left[\hat{A}+\phi_{\varepsilon}\right] \perp \mathcal{C}$ amounts to equating $\mathrm{N} L^{2}$-inner products to zero. This leads to a system of equations to leading order given by (2.11) in the spike points $\left(t_{1}, \ldots, t_{N}\right)$, which can be shown to have a solution.

The main steps in the proof of Theorem 2 can be stated as follows:

The eigenvalue problem is derived by linearizing the Gierer-Meinhardt system (1.1) around the clustered steady state $A_{\varepsilon}$ derived in Theorem 1. It is stated in (8.1) and for $\tau=0$, we have

$$
\tilde{L}_{\varepsilon, t^{\natural}} \phi_{\varepsilon}=\varepsilon^{2} \Delta \phi_{\varepsilon}-\mu(x) \phi_{\varepsilon}+\frac{2 A_{\varepsilon} \phi_{\varepsilon}}{T\left[A_{\varepsilon}\right]}-\frac{A_{\varepsilon}^{2}}{\left(T\left[A_{\varepsilon}\right]\right)^{2}}\left(T^{\prime}\left[A_{\varepsilon}\right] \phi_{\varepsilon}\right)=\lambda_{\varepsilon} \phi_{\epsilon}
$$

where $\lambda_{\varepsilon}$ is some complex number and $\phi_{\varepsilon} \in H^{2}(\Omega)$ satisfying Neumann boundary conditions. Further, for $\phi \in L^{2}(\Omega)$,

$$
T^{\prime}\left[A_{\varepsilon}\right] \phi=2 \xi_{\varepsilon} \int_{\mathbb{R}} G_{D}(\cdot ; z) A_{\varepsilon}(z) \phi(z) d z
$$

is an integral operator solving (8.3).

Then, we consider the eigenvalues in three cases separately as follows:

(1) We first study large eigenvalues $\lambda_{\varepsilon}=O(1)$.

(i) Using (8.1) and the decay of the spikes, it is shown that in leading order an eigenfunction satisfies

$$
\phi_{\varepsilon} \sim \sum_{j=i}^{N} \phi_{\varepsilon, j}\left(\frac{x-t_{j}}{\varepsilon}\right) .
$$

This means that the eigenfunction can be decomposed into parts which are located near each of the spikes.

(ii) Taking the limit $\max \left(D, \frac{\varepsilon}{\sqrt{D}}\right) \rightarrow 0$, we derive $\phi_{\varepsilon, j} \rightarrow \phi_{j}$, where $\phi_{j}$ satisfies the NLEP

$$
\phi_{\varepsilon, j}^{\prime \prime}-\phi_{j}+2 w \phi_{j}-2 \frac{\int_{\mathbb{R}} w \phi_{j} d y}{\int_{\mathbb{R}} w^{2} d y} w^{2}=\frac{\alpha}{\mu^{0}} \phi_{\varepsilon, j}, \quad j=1, \ldots, N
$$

This means that we get an NLEP which has diagonal form.

(iii) By a result in [43], it follows that the spike cluster is stable with respect to large eigenvalues.

(2) Next, we study small eigenvalues $\lambda_{\varepsilon}=o(1)$. 
(i) The ansatz for an eigenfunction is

$$
\phi_{\varepsilon}=\sum_{j=1}^{N} a_{j}^{\varepsilon} \varepsilon \tilde{w}_{\varepsilon, j}^{\prime}(x)+\phi^{\perp}(x)
$$

where $\phi^{\perp} \perp \tilde{w}_{\varepsilon, j}^{\prime}$ in $L^{2}$-sense for $j=1, \ldots, N$.

(ii) Then, we show that $\phi^{\perp}=o(1)$. Note that $\varepsilon \tilde{w}_{\varepsilon, j}^{\prime}$ is of exact order 1 and so it dominates the eigenfunction.

(iii) Taking the spatial derivative of the steady-state problem (4.20), we get an identity for $\tilde{w}_{\varepsilon, j}^{\prime}$ (up to small error terms) which we subtract from the eigenvalue problem.

(iv) We expand the terms in (8.1) around the spikes using the expansion of the Green's function $G_{D}$ and the inhomogeneity $\mu(x)$, both around the spike points, collecting the leading terms and giving rigorous estimates for the remainder.

(v) We multiply the eigenvalue problem (8.1) on both sides by $\varepsilon \tilde{w}_{\varepsilon, k}^{\prime}, k=1, \ldots, N$. Using the results in (iv), we derive the matrix $\mathcal{M}\left(\mathbf{t}^{0}\right)$ stated in (9.13) which determines the stability properties caused by small eigenvalues.

(vi) We determine the eigenvalues and eigenvectors of the matrix $\mathcal{M}\left(\mathbf{t}^{0}\right)$ explicitly by considering it as the finite-difference approximation of a suitable ordinary differential equation, compute the solution of this approximation explicitly and get the eigenvectors by taking its values at uniformly spaced points. See Lemma 13 and its proof.

It follows that the eigenvalue problem is stable with respect to small eigenvalues.

(3) Finally, we show that there are no eigenvalues $\lambda_{\varepsilon}$ with $\left|\lambda_{\varepsilon}\right| \rightarrow \infty$, if we assume that $\operatorname{Re}\left(\lambda_{\varepsilon}\right)>-c$ for some fixed $c>0$. (If the latest condition is not satisfied the eigenfunction will be uniformly bounded in time and so does not cause any instability.)

(i) We multiply (8.1) on both sides by $\phi_{\varepsilon}$. This leads to a quadratic form in $\phi_{\varepsilon}$.

(ii) By explicit estimates, we show that $\left|\lambda_{\varepsilon}\right| \leqslant C$ for some $C>0$. See Remark 14.

\section{Preliminaries: scaling property, Green's function and eigenvalue problems}

In this section, we will provide some preliminaries which will be needed later for the existence and stability proofs.

Let $w$ be the ground state solution given in (2.1). By a simple scaling argument, the function

$$
w_{a}(y)=a w(\sqrt{a} y)
$$

is the unique solution of the problem

$$
\left\{\begin{array}{l}
w_{a}^{\prime \prime}-a w_{a}+w_{a}^{2}=0, \quad y \in \mathbb{R}, \\
w_{a}>0, \quad w_{a}(0)=\max _{y \in \mathbb{R}} w_{a}(y), \quad w_{a}(y) \rightarrow 0 \text { as }|y| \rightarrow \infty .
\end{array}\right.
$$


We compute

$$
\begin{gathered}
\int_{\mathbb{R}} w_{a}^{2}(y) d y=a^{3 / 2} \int_{\mathbb{R}} w^{2}(z) d z, \int_{\mathbb{R}} w_{a}^{3}(y) d y=a^{5 / 2} \int_{\mathbb{R}} w^{3}(z) d z, \\
\int_{\mathbb{R}}\left(w_{a}^{\prime}\right)^{2}(y) d y=a^{5 / 2} \int_{\mathbb{R}}\left(w^{\prime}\right)^{2}(z) d z .
\end{gathered}
$$

For $z \in(-1,1)$, let $G_{D}(x, z)$ be the Green's function defined by

$$
\left\{\begin{array}{l}
D G_{D}^{\prime \prime}(x, z)-G_{D}(x, z)+\delta_{z}(x)=0, x \in(-1,1), \\
G_{D}^{\prime}(-1, z)=G_{D}^{\prime}(1, z)=0
\end{array}\right.
$$

where $G_{D}^{\prime}(x, z)=\frac{\partial}{\partial x} G_{D}(x, z)$ (and the left-hand and right-hand limits are considered for $x=z$ ). We calculate

$$
G_{D}(x, z)= \begin{cases}\frac{\theta}{\sinh (2 \theta)} \cosh [\theta(1+x)] \cosh [\theta(1-z)], & -1<x<z \\ \frac{\theta}{\sinh (2 \theta)} \cosh [\theta(1-x)] \cosh [\theta(1+z)], & z<x<1\end{cases}
$$

where

$$
\theta=D^{-1 / 2}
$$

We set

$$
K_{D}(|x-z|)=\frac{1}{2 \sqrt{D}} e^{-\frac{1}{\sqrt{D}}|x-z|}
$$

to be the singular part of $G_{D}(x, z)$. Let the regular part $H_{D}$ of $G_{D}$ be defined by $H_{D}=K_{D}-G_{D}$. Note that $H_{D}(x, z)$ belongs to the space $C^{\infty}((-1,1) \times(-1,1))$.

By (3.5), we have

$$
G_{D}\left(t^{0}, t^{0}\right)=K_{D}(0)\left(1+O\left(e^{-2\left(d_{0}-\eta_{0}\right) / \sqrt{D}}\right)\right)
$$

where $d_{0}=1-\left|t^{0}\right|$ and $\eta_{0}>0$ is an arbitrary but fixed constant.

For $\hat{\xi}^{0}$, we estimate

$$
\hat{\xi}^{0}=\frac{1}{2 \sqrt{D} G_{D}\left(t^{0}, t^{0}\right)\left(\mu^{0}\right)^{3 / 2}}=\frac{1}{\left(\mu^{0}\right)^{3 / 2}}+O\left(e^{-2\left(d_{0}-\eta_{0}\right) / \sqrt{D}}\right)
$$

by (3.6), (3.7).

Let us denote $\frac{\partial}{\partial t_{i}}$ as $\nabla_{t_{i}}$. When $i \neq j$, we can define $\nabla_{t_{i}} G\left(t_{i}, t_{j}\right)$ in the classical way because the function is smooth. When $i=j$, then $K_{D}\left(\left|t_{i}-t_{j}\right|\right)=K_{D}(0)=\frac{1}{2 \sqrt{D}}$ is a constant independent of $t_{i}$ and we define

$$
\nabla_{t_{i}} G_{D}\left(t_{i}, t_{i}\right):=-\left.\frac{\partial}{\partial x}\right|_{x=t_{i}} H\left(x, t_{i}\right)
$$


Similarly, we define

$$
\nabla_{t_{i}} \nabla_{t_{j}} G_{D}\left(t_{i}, t_{j}\right)=\left\{\begin{array}{l}
-\left.\left.\frac{\partial}{\partial x}\right|_{x=t_{i}} \frac{\partial}{\partial y}\right|_{y=t_{i}} H_{D}(x, y) \text { if } i=j \\
\nabla_{t_{i}} \nabla_{t_{j}} G_{D}\left(t_{i}, t_{j}\right) \text { if } i \neq j
\end{array}\right.
$$

For convenience and clarity, we introduce a re-scaled version of the Green's function which has a finite limit as $D \rightarrow 0$. Thus, we set

$$
\begin{gathered}
\hat{G}_{D}(x, z)=2 \sqrt{D} G_{D}(x, z), \\
\hat{K}_{D}(x, z)=2 \sqrt{D} K_{D}(|x-z|), \\
\hat{H}_{D}(x, z)=2 \sqrt{D} H_{D}(x, z) .
\end{gathered}
$$

Throughout the paper, let $C, c$ denote generic constants which may change from line to line.

\section{Existence proof I: approximate solutions}

Let $t^{0} \in(-1,1)$ be a non-degenerate local minimum point of the precursor inhomogeneity, i.e. we assume that (2.7) is satisfied. In this section, we construct an approximation to a spike cluster solution to (1.2) which concentrates at $t^{0}$.

The approximate cluster consists of the spikes $\mu_{i} w\left(\sqrt{\mu_{i}} \frac{x-t_{i}}{\varepsilon}\right)$ which are centred at the points $t_{i}$ and have the scaling factors

$$
\mu_{i}=\mu\left(t_{i}\right), \quad \text { where } i=1, \ldots, N \text {. }
$$

Let $\Omega_{\eta}$ denote the set of all $\mathbf{t}=\left(t_{1}, t_{2}, \ldots, t_{N}\right) \in \Omega^{N}$ with $-1<t_{1}<t_{2} \cdots<t_{N}<1$ satisfying (4.2) and (4.3), where

$$
\left.\mid \frac{t_{s}-t_{s-1}}{\sqrt{D}}-\log \frac{1}{D}+\log \log \frac{1}{D}+\log \left(\frac{5 \mu^{\prime \prime}\left(t^{0}\right)}{16 \mu^{0}}\right)+\log [(s-1)(N+1-s)]\right] \mid \leqslant \eta
$$

for $s=2, \ldots, N$,

$$
\left|\frac{1}{N} \sum_{k=1}^{N} t_{k}-t^{0}\right| \leqslant \eta \log \frac{1}{D}
$$

and $\eta>0$ is a constant which is small enough and will be chosen in Section 7 (see equation (7.9)). The reason for assuming (4.2) and (4.3) will become clear in Section 7 when we solve the reduced problem.

We further denote

$$
\mathbf{t}^{0}=\left(t^{0}, t^{0}, \ldots, t^{0}\right)
$$

and set

$$
\Omega_{0}=\left\{\mathbf{t}^{0}\right\}
$$


To simplify our notation, for $\mathbf{t} \in \Omega_{\eta}$ and $k=1, \ldots, N$, we set

$$
w_{k}(x)=\mu_{k} w\left(\sqrt{\mu_{k}} \frac{x-t_{i}}{\varepsilon}\right)
$$

and

$$
\tilde{w}_{k}(x)=\mu_{k} w\left(\sqrt{\mu_{k}} \frac{x-t_{k}}{\varepsilon}\right) \cdot \chi\left(\left|\frac{x-t_{k}}{\delta_{\varepsilon}}\right|\right),
$$

where $\chi$ is a smooth cut-off function which satisfies the conditions

$$
\chi(x)=1 \text { for }|x|<\frac{1}{2}, \quad \chi(x)=0 \text { for }|x|>\frac{3}{4}, \quad \chi \in C_{0}^{\infty}(\mathbb{R})
$$

and

$$
\varepsilon \ll \delta_{\varepsilon} \ll \frac{20}{\sqrt{\mu^{0}}} \varepsilon \log \frac{1}{\varepsilon} .
$$

Using (4.2), we have $\left|t_{i}-t^{0}\right|=O\left(\sqrt{D} \log \frac{1}{D}\right)$ for $i=1, \ldots, N$. This implies

$$
\begin{gathered}
\left|\mu\left(t_{i}\right)-\mu\left(t^{0}\right)\right|=O\left(D\left(\log \frac{1}{D}\right)^{2}\right) \\
\mu^{\prime}\left(t_{i}\right)=\mu^{\prime \prime}\left(t^{0}\right)\left(t_{i}-t^{0}\right)+O\left(D\left(\log \frac{1}{D}\right)^{2}\right)=O\left(\sqrt{D} \log \frac{1}{D}\right), \\
\mu^{\prime \prime}\left(t_{i}\right)=\mu^{\prime \prime}\left(t^{0}\right)+O\left(\sqrt{D} \log \frac{1}{D}\right)=O(1), \mu^{\prime \prime}\left(t^{0}\right)=O(1) . \\
\mu^{\prime \prime \prime}\left(t_{i}\right)=O(1), \mu^{\prime \prime \prime}\left(t^{0}\right)=O(1) .
\end{gathered}
$$

Further, we compute, using (3.5),

$$
\hat{G}_{D}\left(t_{i}, t_{i}\right)=\hat{K}_{D}(0)\left(1+O\left(e^{-2\left(d_{0}-\eta_{0}\right) / \sqrt{D}}\right)\right)=1+O\left(e^{-2\left(d_{0}-\eta_{0}\right) / \sqrt{D}}\right),
$$

where $d_{0}=\min \left(1-t^{0}, t^{0}+1\right), \eta_{0}>0$ is an arbitrary but fixed constant (compare (3.7)). We have

$$
\begin{gathered}
\hat{G}_{D}\left(t_{i}, t_{s}\right)=O\left(D \log \frac{1}{D}\right), \hat{K}_{D}\left(t_{i}, t_{s}\right)=O\left(D \log \frac{1}{D}\right) \text { for }|i-s|=1, \\
\hat{G}_{D}\left(t_{i}, t_{s}\right)=O\left(\left(D \log \frac{1}{D}\right)^{2}\right), \hat{K}_{D}\left(t_{i}, t_{s}\right)=O\left(\left(D \log \frac{1}{D}\right)^{2}\right) \text { for }|i-s|=2 .
\end{gathered}
$$

Generally, we have

$$
\hat{G}_{D}\left(t_{i}, t_{s}\right)=O\left(\left(D \log \frac{1}{D}\right)^{|i-s|}\right), \hat{K}_{D}\left(t_{i}, t_{s}\right)=O\left(\left(D \log \frac{1}{D}\right)^{|i-s|}\right) \text { for }|i-s| \geqslant 1
$$


For the derivatives, we estimate

$$
\begin{aligned}
& \frac{\partial^{k}}{\partial t_{i}^{k}} \hat{G}_{D}\left(t_{i}, t_{s}\right)=O\left(\left(D \log \frac{1}{D}\right)^{|i-s|} D^{-k / 2}\right) \text { for }|i-s| \geqslant 1, \quad k=1,2, \ldots \\
& \frac{\partial^{k}}{\partial t_{i}^{k}} \hat{K}_{D}\left(t_{i}, t_{s}\right)=O\left(\left(D \log \frac{1}{D}\right)^{|i-s|} D^{-k / 2}\right) \text { for }|i-s| \geqslant 1, \quad k=1,2, \ldots
\end{aligned}
$$

and analogous results hold for the mixed derivatives.

By rescaling $\hat{A}=\xi_{\varepsilon} A, \hat{H}=\xi_{\varepsilon} H$ with $\xi_{\varepsilon}$ defined in (2.6), it follows that (1.2) is equivalent to the following system for the re-scaled functions $\hat{A}, \hat{H}$ :

$$
\left\{\begin{array}{l}
\varepsilon^{2} \hat{A}^{\prime \prime}-\mu(x) \hat{A}+\frac{\hat{A}^{2}}{\hat{H}}=0, \quad x \in(-1,1), \\
D \hat{H}^{\prime \prime}-\hat{H}+\xi_{\varepsilon} \hat{A}^{2}=0, \quad x \in(-1,1), \\
\hat{A}(x)>0, \hat{H}(x)>0 \quad \text { in } \quad(-1,1), \\
\hat{A}^{\prime}(-1)=\hat{A}^{\prime}(1)=\hat{H}^{\prime}(-1)=\hat{H}^{\prime}(1)=0 .
\end{array}\right.
$$

From now on, we shall work with (4.20) and drop the hats. Next, we rewrite (4.20) as a single equation with a non-local term.

For a function $A \in H^{2}(-1,1)$, we define $T[A]$ to be the solution of

$$
\left\{\begin{array}{l}
D(T[A])^{\prime \prime}-T[A]+\xi_{\varepsilon} A^{2}=0,-1<x<1, \\
(T[A])^{\prime}(-1)=(T[A])^{\prime}(1)=0 .
\end{array}\right.
$$

It is easy to see that the solution $T[A]$ is unique and positive. Then, (4.20) becomes

$$
\mathcal{S}_{\varepsilon}[A]:=\varepsilon^{2} A^{\prime \prime}-\mu(x) A+\frac{A^{2}}{T[A]}=0, \quad A>0, \quad A^{\prime}(-1)=A^{\prime}(1)=0 .
$$

For $\mathbf{t} \in \Omega_{\eta}$, we define an approximate solution to (4.22) by the ansatz

$$
A(x)=w_{\varepsilon, \mathbf{t}}(x)=\sum_{k=1}^{N} \hat{\xi}_{k} \tilde{w}_{k}(x), \quad x \in \Omega,
$$

where $\mathbf{t} \in \Omega_{\eta}, \tilde{w}_{k}$ has been defined in (4.7) and $\hat{\xi}_{k}$ satisfies the amplitude identity

$$
\hat{\xi}_{s}=T[A]\left(t_{s}\right)
$$

Intuitively, this ansatz is close to a solution of (4.22) since by the choice of $\tilde{w}_{k}$, the first equation is approximately satisfied and due to (4.24) the second equation holds approximately.

Next, we are now going to determine the amplitudes $\hat{\xi}_{k}$ to leading order so that $(4.24)$ will be satisfied. 
From (4.21), we have

$$
\begin{aligned}
T\left[w_{\varepsilon, \mathbf{t}}\right]\left(t_{s}\right) & =\xi_{\varepsilon} \int_{-1}^{1} G_{D}\left(t_{s}, z\right) w_{\varepsilon, \mathbf{t}}^{2}(z) d z \\
& =\xi_{\varepsilon} \int_{-1}^{1} G_{D}\left(t_{s}, z\right)\left[\sum_{k=1}^{N} \hat{\xi}_{k}^{2} \tilde{w}_{k}^{2}(z)+\sum_{k \neq l} \hat{\xi}_{k} \hat{\xi}_{l} \tilde{w}_{k}(z) \tilde{w}_{l}(z)\right] d z
\end{aligned}
$$

We have

$$
\xi_{\varepsilon} \int_{\Omega} G_{D}\left(t_{s}, x\right) \tilde{w}_{k}^{2}(x) d x=\xi_{\varepsilon} \int_{\Omega} G_{D}\left(t_{s}, x\right)\left(\mu_{k} w\left(\sqrt{\mu_{k}} \frac{x-t_{k}}{\varepsilon}\right)\right)^{2} d x\left(1+O\left(\varepsilon^{10}\right)\right) .
$$

For $k \neq s$, we compute

$$
\begin{aligned}
\xi_{\varepsilon} \int_{\Omega} G_{D}\left(t_{s}, x\right) \tilde{w}_{k}^{2}(x) d x & =\xi_{\varepsilon} \varepsilon\left(\mu_{k}\right)^{3 / 2} G_{D}\left(t_{s}, t_{k}\right)\left[\int_{\mathbb{R}} w^{2}(y) d y+O\left(\frac{\varepsilon}{\sqrt{D}}\right)\right] \\
& =\left(\mu_{k}\right)^{3 / 2} \hat{G}_{D}\left(t_{s}, t_{k}\right)\left[1+O\left(\frac{\varepsilon}{\sqrt{D}}\right)\right] \\
& =\left(\mu^{0}\right)^{3 / 2} \hat{G}_{D}\left(t_{s}, t_{k}\right)\left[1+O\left(\frac{\varepsilon}{\sqrt{D}}+D\left(\log \frac{1}{D}\right)^{2}\right)\right] \\
& =\left(\mu^{0}\right)^{3 / 2} O\left(D \log \frac{1}{D}\right)\left[1+O\left(\frac{\varepsilon}{\sqrt{D}}+D\left(\log \frac{1}{D}\right)^{2}\right)\right] \\
& =O\left(D \log \frac{1}{D}\right)
\end{aligned}
$$

using (2.6), (3.10), (4.10) and (4.17). For $k=s$, we have

$$
\begin{aligned}
\xi_{\varepsilon} \int_{\Omega} G_{D}\left(t_{s}, x\right) \tilde{w}_{s}^{2}(x) d x & =\xi_{\varepsilon} \int_{\Omega}\left[\frac{1}{2 \sqrt{D}} e^{-\left|t_{s}-x\right| / \sqrt{D}}-H_{D}\left(t_{s}, x\right)\right] \tilde{w}_{s}^{2}(x) d x \\
& =\xi_{\varepsilon} \varepsilon\left(\mu_{s}\right)^{3 / 2} G_{D}\left(t_{s}, t_{s}\right)\left[\int_{\mathbb{R}} w^{2}(y) d y+O\left(\frac{\varepsilon}{\sqrt{D}}\right)\right] \\
& =\left(\mu_{s}\right)^{3 / 2} \hat{G}_{D}\left(t_{s}, t_{s}\right)+O\left(\frac{\varepsilon}{\sqrt{D}}\right) \\
& =\left(\mu^{0}\right)^{3 / 2} \hat{G}_{D}\left(t_{s}, t_{s}\right)+O\left(\frac{\varepsilon}{\sqrt{D}}+D\left(\log \frac{1}{D}\right)^{2}\right)
\end{aligned}
$$

using (2.6), (3.10) and (4.10). Next, for $k \neq l$, we have

$$
\xi_{\varepsilon} \int_{\Omega} \hat{G}_{D}\left(t_{s}, z\right) \tilde{w}_{k}(z) \tilde{w}_{l}(z) d z=0
$$

by (4.7). Combining (4.26)-(4.28), we have

$$
T\left[w_{\varepsilon, \mathbf{t}}\right]\left(t_{s}\right)=\hat{\xi}_{s}^{2}\left(\mu^{0}\right)^{3 / 2}\left[1+O\left(\frac{\varepsilon}{\sqrt{D}}+D\left(\log \frac{1}{D}\right)^{2}\right)\right]
$$


using (3.6), (3.7), (3.10). Then, (4.29) has a unique solution which satisfies

$$
\hat{\xi}_{s}=\frac{1}{\left(\mu^{0}\right)^{3 / 2}}\left[1+O\left(\frac{\varepsilon}{\sqrt{D}}+D\left(\log \frac{1}{D}\right)^{2}\right)\right] .
$$

This concludes the construction of the approximate solution.

In the section, we will compute its error.

\section{Existence proof II - error of approximate solution}

In this section, we compute the error terms caused by the approximate solutions in Section 4. We begin by considering the spatial dependence of the inhibitor near the spikes which is given by the difference $T\left[w_{\varepsilon, \mathbf{t}}\right]\left(x_{s}\right)-T\left[w_{\varepsilon, \mathbf{t}}\right]\left(t_{s}\right)$ for $\mathbf{x}=\left(x_{1}, \ldots, x_{N}\right) \in \Omega_{\eta}$ and $\mathbf{t} \in \Omega_{\eta}$, where the non-local operator $\mathcal{T}[A]$ has been defined in (4.21) and the approximate solution has been introduced in (4.23).

To simplify our notation, we let

$$
H_{\varepsilon, \mathbf{t}}\left(x_{s}\right)=T\left[w_{\varepsilon, \mathbf{t}}\right]\left(x_{s}\right)
$$

Let $x_{s}=t_{s}+\varepsilon y$. We calculate

$$
\begin{aligned}
& H_{\varepsilon, \mathbf{t}}\left(t_{s}+\varepsilon y\right)-H_{\varepsilon, \mathbf{t}}\left(t_{s}\right) \\
& \quad=\xi_{\varepsilon} \int_{\Omega}\left[G_{D}\left(t_{s}+\varepsilon y, x\right)-G_{D}\left(t_{s}, x\right)\right]\left(\sum_{k=1}^{N} \hat{\xi}_{k}^{2} \tilde{w}_{k}^{2}(x)+\sum_{k \neq l} \hat{\xi}_{k} \hat{\xi}_{l} \tilde{w}_{k}(x) \tilde{w}_{l}(x)\right) d x, \\
& \quad=J_{1},
\end{aligned}
$$

where $\xi_{\varepsilon}$ has been introduced in (2.6) and $J_{1}$ is defined by the latest equality. For $J_{1}$, we have

$$
\begin{aligned}
J_{1} & =\xi_{\varepsilon} \int_{\Omega}\left[G_{D}\left(t_{s}+\varepsilon y, x\right)-G_{D}\left(t_{s}, x\right)\right]\left(\sum_{k=1}^{N} \hat{\xi}_{k}^{2} \tilde{w}_{k}^{2}(x)+\sum_{k \neq l} \hat{\xi}_{k} \hat{\xi}_{l} \tilde{w}_{k}(x) \tilde{w}_{l}(x)\right) d x \\
& =\xi_{\varepsilon} \sum_{k=1}^{N} \hat{\xi}_{k}^{2} \int_{\Omega}\left[G_{D}\left(t_{s}+\varepsilon y, x\right)-G_{D}\left(t_{s}, x\right)\right] \tilde{w}_{k}^{2} d x .
\end{aligned}
$$

by (4.7). We further compute

$$
\begin{aligned}
\xi_{\varepsilon} & \int_{\Omega}\left[G_{D}\left(t_{s}+\varepsilon y, x\right)-G_{D}\left(t_{s}, x\right)\right] \tilde{w}_{k}^{2} d x \\
& =\xi_{\varepsilon} \int_{\Omega}\left[\frac{1}{2 \sqrt{D}}\left(e^{-\left|t_{s}+\varepsilon y-x\right| / \sqrt{D}}-e^{-\left|t_{s}-x\right| / \sqrt{D}}\right)-\left(H_{D}\left(t_{s}+\varepsilon y, x\right)-H_{D}\left(t_{s}, x\right)\right)\right] \tilde{w}_{k}^{2} d x \\
& =\xi_{\varepsilon} \frac{1}{2 \sqrt{D}} \int_{\Omega}\left(e^{-\left|t_{s}+\varepsilon y-x\right| / \sqrt{D}}-e^{-\left|t_{s}-x\right| / \sqrt{D}}\right) \tilde{w}_{k}^{2} d x\left(1+O\left(e^{-2\left(d_{0}-\eta_{0}\right) / \sqrt{D}}\right)\right)
\end{aligned}
$$


using (4.14). Let $x=t_{k}+\varepsilon \tilde{z}$. For $k=s$, we have

$$
\begin{aligned}
\xi_{\varepsilon} & \frac{1}{2 \sqrt{D}} \int_{\Omega}\left(e^{-\left|t_{s}+\varepsilon y-x\right| / \sqrt{D}}-e^{-\left|t_{s}-x\right| / \sqrt{D}}\right) \tilde{w}_{s}^{2}(x) d x \\
& =\xi_{\varepsilon} \frac{\varepsilon}{2 \sqrt{D}} \int_{\mathbb{R}}\left(e^{-\varepsilon|y-\tilde{z}| / \sqrt{D}}-e^{-\varepsilon|\tilde{z}| / \sqrt{D}}\right) w_{\mu_{s}}^{2}(\tilde{z}) d \tilde{z}\left(1+O\left(\varepsilon^{10}\right)\right) \\
& =\xi_{\varepsilon} \frac{\varepsilon}{2 \sqrt{D}}\left[\frac{\varepsilon}{\sqrt{D}} \int_{\mathbb{R}}(|\tilde{z}|-|y-\tilde{z}|) w_{\mu_{s}}^{2}(\tilde{z}) d \tilde{z}+O\left(\frac{\varepsilon^{2}}{D} y^{2}\right)\right]\left(1+O\left(\varepsilon^{10}\right)\right) \\
& =\xi_{\varepsilon} \frac{\varepsilon}{2 \sqrt{D}}\left[\frac{\varepsilon}{\sqrt{D}} \int_{\mathbb{R}}(|\tilde{z}|-|y-\tilde{z}|) w_{\mu^{0}}^{2}(\tilde{z}) d \tilde{z}+O\left(\frac{\varepsilon^{2}}{D} y^{2}\right)\right]\left(1+O\left(D\left(\log \frac{1}{D}\right)^{2}+\varepsilon^{10}\right)\right) \\
& =\frac{1}{\int_{\mathbb{R}} w^{2}(\tilde{y}) d \tilde{y}}\left[\frac{\varepsilon}{\sqrt{D}} T_{0}(y)+O\left(\frac{\varepsilon^{2}}{D} y^{2}\right)\right]\left(1+O\left(D\left(\log \frac{1}{D}\right)^{2}+\varepsilon^{10}\right)\right),
\end{aligned}
$$

where $w_{\mu_{s}}$ has been defined in (3.1) and

$$
T_{0}(y)=\int_{\mathbb{R}}(|\tilde{z}|-|y-\tilde{z}|) w_{\mu^{0}}^{2} d \tilde{z}
$$

is an even function, using (2.6) and (4.10). For $k \neq s$, we have

$$
\begin{aligned}
& \xi_{\varepsilon} \frac{1}{2 \sqrt{D}} \int_{\Omega}\left(e^{-\left|t_{s}+\varepsilon y-x\right| / \sqrt{D}}-e^{-\left|t_{s}-x\right| / \sqrt{D}}\right) \tilde{w}_{k}^{2}(x) d x \\
& =\xi_{\varepsilon} \frac{\varepsilon}{2 \sqrt{D}} \int_{\mathbb{R}}\left(e^{-\left|t_{s}-t_{k}+\varepsilon(y-\tilde{z})\right| / \sqrt{D}}-e^{-\left|t_{s}-t_{k}-\varepsilon \tilde{z}\right| / \sqrt{D}}\right) w_{\mu_{k}}^{2}(\tilde{z}) d \tilde{z}\left(1+O\left(\varepsilon^{10}\right)\right) \\
& =\xi_{\varepsilon} \frac{\varepsilon}{2 \sqrt{D}}\left(\mu_{k}\right)^{3 / 2}\left[e^{-\left|t_{s}-t_{k}\right| / \sqrt{D}}\left(-\frac{t_{s}-t_{k}}{\left|t_{s}-t_{k}\right|}\right) \frac{\varepsilon y}{\sqrt{D}}+O\left(D \log \frac{1}{D} \frac{\varepsilon^{2}}{D} y^{2}\right)\right] \\
& \times \int_{\mathbb{R}} w^{2}(\tilde{z}) d \tilde{z}\left(1+O\left(\varepsilon^{10}\right)\right) \\
& =\left(\mu^{0}\right)^{3 / 2}\left[e^{-\left|t_{s}-t_{k}\right| / \sqrt{D}}\left(-\frac{t_{s}-t_{k}}{\left|t_{s}-t_{k}\right|}\right) \frac{\varepsilon y}{\sqrt{D}}+O\left(\varepsilon^{2} \log \frac{1}{D} y^{2}\right)\right] \\
& \times\left(1+O\left(D\left(\log \frac{1}{D}\right)^{2}+\varepsilon^{10}\right)\right)
\end{aligned}
$$

using (4.10) and (2.6). Combining (5.5) and (5.7), we have

$$
\begin{aligned}
& H_{\varepsilon, \mathbf{t}}\left(t_{s}+\varepsilon y\right)-H_{\varepsilon, \mathbf{t}}\left(t_{s}\right) \\
& =\left(\hat{\xi}_{s}^{2} \frac{1}{\int_{\mathbb{R}} w^{2}(\tilde{y}) d \tilde{y} \frac{\varepsilon}{\sqrt{D}} \int_{\mathbb{R}}(|\tilde{z}|-|y-\tilde{z}|) w_{\mu_{s}}^{2}(\tilde{z}) d \tilde{z}+O\left(\frac{\varepsilon^{2}}{D} y^{2}\right)}\right. \\
& \left.\quad+\sum_{k \neq s} \hat{\xi}_{k}^{2}\left(\mu_{k}\right)^{3 / 2} e^{-\left|t_{s}-t_{k}\right| / \sqrt{D}}\left(-\frac{t_{s}-t_{k}}{\left|t_{s}-t_{k}\right|}\right) \frac{\varepsilon y}{\sqrt{D}}+O\left(\varepsilon^{2} \log \frac{1}{D} y^{2}\right)\right) \\
& \quad \times\left(1+O\left(D\left(\log \frac{1}{D}\right)^{2}+\varepsilon^{10}\right)\right) .
\end{aligned}
$$




\section{Remark 7}

(i) The second line in (5.8) is an even function in the inner variable $y$ which will drop out in many subsequent computations due to symmetry.

(ii) The third line in (5.8) is an odd function in the inner variable $y$. For $\mathbf{t} \in \Omega_{\eta}$, we have

$$
\begin{gathered}
e^{-\left|t_{s}-t_{k}\right| / \sqrt{D}}=O\left(D \log \frac{1}{D}\right), \quad|k-s|=1, \\
e^{-\left|t_{s}-t_{k}\right| / \sqrt{D}}=O\left(\left(D \log \frac{1}{D}\right)^{2}\right), \quad|k-s| \geqslant 2 .
\end{gathered}
$$

Thus, the third line in (5.8) is of exact order $O\left(\varepsilon \sqrt{D} \log \frac{1}{D} y\right)$.

Next, we compute and estimate the error terms of the Gierer-Meinhardt system (4.20) for the approximate solution $w_{\varepsilon, \text { t }}$. We recall that a steady state for $(4.20)$ is given by $\mathcal{S}_{\varepsilon}[A]=0$, where

$$
\mathcal{S}_{\varepsilon}[A]:=\varepsilon^{2} A^{\prime \prime}-\mu(x) A+\frac{A^{2}}{T[A]}
$$

and $T[A]$ is defined by (4.21), combined with Neumann boundary conditions $A^{\prime}(-1)=$ $A^{\prime}(1)=0$. We now compute the error term

$$
\begin{aligned}
& \mathcal{S}_{\varepsilon}\left[w_{\varepsilon, \mathbf{t}}\right]=\mathcal{S}_{\varepsilon}\left[\sum_{s=1}^{N} \hat{\xi}_{s} \tilde{w}_{s}\right] \\
&=\varepsilon^{2} \Delta\left(\sum_{s=1}^{N} \hat{\xi}_{s} \tilde{w}_{s}\right)-\mu(x) \sum_{s=1}^{N} \hat{\xi}_{s} \tilde{w}_{s}+\frac{\left(\sum_{s=1}^{N} \hat{\xi}_{s} \tilde{w}_{s}\right)^{2}}{H_{\varepsilon, \mathbf{t}}} \\
&=\left[\sum_{s=1}^{N}\left(\varepsilon^{2} \Delta\left(\hat{\xi}_{s} \tilde{w}_{s}\right)-\mu_{s} \hat{\xi}_{s} \tilde{w}_{s}+\frac{\left(\hat{\xi}_{s} \tilde{w}_{s}\right)^{2}}{H_{\varepsilon, \mathbf{t}}\left(t_{s}\right)}\right)-\sum_{s=1}^{N}\left(\mu(x)-\mu\left(t_{s}\right)\right) \hat{\xi}_{s} \tilde{w}_{s}\right. \\
&\left.-\sum_{s=1}^{N} \frac{\left(\hat{\xi}_{s} \tilde{w}_{s}\right)^{2}}{\left(H_{\varepsilon, \mathbf{t}}\left(t_{s}\right)\right)^{2}}\left[H_{\varepsilon, \mathbf{t}}(x)-H_{\varepsilon, \mathbf{t}}\left(t_{s}\right)\right]\left(1+O\left(\frac{\varepsilon}{\sqrt{D}}|y|\right)\right)\right]\left(1+O\left(e^{-2\left(d_{0}-\eta_{0}\right) / \sqrt{D}}\right)\right) .
\end{aligned}
$$

Using the amplitude identity $\hat{\xi}_{k}=T[A]\left(t_{k}\right)$ and the equation (3.2) of the spike profile for $a=\mu_{s}$, we have

$$
\begin{aligned}
\mathcal{S}_{\varepsilon}\left[w_{\varepsilon, \mathbf{t}}\right]= & {\left[-\sum_{s=1}^{N}\left(\mu^{\prime}\left(t_{s}\right) \varepsilon y\right) \hat{\xi}_{s} w_{\mu^{s}}\right.} \\
& -\sum_{s=1}^{N} \hat{\xi}_{s}^{2} w_{\mu^{s}}^{2} \frac{1}{\int_{\mathbb{R}} w^{2}(\tilde{y}) d \tilde{y}} \frac{\varepsilon}{\sqrt{D}} \int_{\mathbb{R}}(|\tilde{z}|-|y-\tilde{z}|) w_{\mu_{s}}^{2}(\tilde{z}) d \tilde{z}
\end{aligned}
$$




$$
\begin{aligned}
& \left.+\sum_{s=1}^{N} w_{\mu^{s}}^{2} \sum_{k \neq s} \hat{\xi}_{k}^{2}\left(\mu_{k}\right)^{3 / 2} e^{-\left|t_{s}-t_{k}\right| / \sqrt{D}}\left(-\frac{t_{s}-t_{k}}{\left|t_{s}-t_{k}\right|}\right) \frac{\varepsilon y}{\sqrt{D}}\right] \\
& \quad \times\left(1+O\left(D\left(\log \frac{1}{D}\right)^{2}+\varepsilon^{10}\right)\right) .
\end{aligned}
$$

Using $\mu^{\prime}\left(t^{0}\right)=0$, we get

$$
\begin{aligned}
\mathcal{S}_{\varepsilon}\left[w_{\varepsilon, \mathbf{t}}\right]= & {\left[-\sum_{s=1}^{N}\left(\mu^{\prime \prime}\left(t^{0}\right)\left(t_{s}-t^{0}\right) \varepsilon y\right) \hat{\xi}_{s} w_{\mu_{s}}\left(1+O(\varepsilon|y|)+O\left(\sqrt{D} \log \frac{1}{D}\right)\right)\right.} \\
& -\sum_{s=1}^{N} \hat{\xi}_{s}^{2} w_{\mu^{s}}^{2} \frac{1}{\int_{\mathbb{R}} w^{2}(\tilde{y}) d \tilde{y}} \frac{\varepsilon}{\sqrt{D}} \int_{\mathbb{R}}(|\tilde{z}|-|y-\tilde{z}|) w_{\mu_{s}}^{2}(\tilde{z}) d \tilde{z} \\
& \left.+\sum_{s=1}^{N} w_{\mu^{s}}^{2} \sum_{k \neq s} \hat{\xi}_{k}^{2}\left(\mu_{k}\right)^{3 / 2} e^{-\left|t_{s}-t_{k}\right| / \sqrt{D}}\left(-\frac{t_{s}-t_{k}}{\left|t_{s}-t_{k}\right|}\right) \frac{\varepsilon y}{\sqrt{D}}\left(1+O\left(\frac{\varepsilon}{\sqrt{D}}|y|\right)\right)\right] \\
& \times\left(1+O\left(D\left(\log \frac{1}{D}\right)^{2}+\varepsilon^{10}\right)\right)
\end{aligned}
$$

Now, we readily have the estimate

$$
\left\|\mathcal{S}_{\varepsilon}\left[w_{\varepsilon, \mathbf{t}}\right]\right\|_{L^{2}\left(-\frac{1}{\varepsilon}, \frac{1}{\varepsilon}\right)}=O\left(\frac{\varepsilon}{\sqrt{D}}\right) .
$$

Remark 8 The estimates derived in this section will be needed to conclude the existence proof using Liapunov-Schmidt reduction in Section 6. In particular, they will imply an explicit formula for the positions of the spikes in Section 7.

\section{Existence proof III - Liapunov-Schmidt reduction}

In this section, we study the linear operator defined by

$$
\begin{gathered}
\tilde{L}_{\varepsilon, \mathbf{t}}:=\mathcal{S}_{\epsilon}^{\prime}[A] \phi=\varepsilon^{2} \Delta \phi-\mu(x) \phi+\frac{2 A \phi}{T[A]}-\frac{A^{2}}{(T[A])^{2}}\left(T^{\prime}[A] \phi\right), \\
\tilde{L}_{\varepsilon, \mathbf{t}}: H^{2}(\Omega) \rightarrow L^{2}(\Omega),
\end{gathered}
$$

where $A=w_{\varepsilon, \mathrm{t}}$ and $T^{\prime}[A]$ has been defined in (8.3).

We will prove results on its invertibility after suitable projections. This will have important implications on the existence of solutions of the non-linear problem including bounds in suitable norms. The proof uses the method of Liapunov-Schmidt reduction which was also considered in $[10,12,13,25,26,37]$ and other works. 
We define the approximate kernel and co-kernel of the operator $\tilde{L}_{\varepsilon, t}$, respectively, as follows:

$$
\begin{aligned}
\mathcal{K}_{\varepsilon, \mathbf{t}} & :=\operatorname{span}\left\{\frac{d \tilde{w}_{i}}{d x} \mid i=1, \ldots, N\right\} \subset H^{2}(\Omega), \\
\mathcal{C}_{\varepsilon, \mathbf{t}} & :=\operatorname{span}\left\{\frac{d \tilde{w}_{i}}{d x} \mid i=1, \ldots, N\right\} \subset L^{2}(\Omega) .
\end{aligned}
$$

Recall that the vectorial linear operator $L$ has been introduced in (A 2) as follows:

$$
L \Phi:=\Delta \Phi-\Phi+2 w \Phi-2 \frac{\int_{\mathbb{R}} w \Phi}{\int_{\mathbb{R}} w^{2}} w^{2},
$$

where

$$
\Phi=\left(\begin{array}{l}
\phi_{1} \\
\phi_{2} \\
\vdots \\
\phi_{N}
\end{array}\right) \in\left(H^{2}(\mathbb{R})\right)^{N}
$$

By Lemma 21, we know that

$$
L:\left(X_{0} \oplus \cdots \oplus X_{0}\right)^{\perp} \cap\left(H^{2}(\mathbb{R})\right)^{N} \rightarrow\left(X_{0} \oplus \cdots \oplus X_{0}\right)^{\perp} \cap\left(L^{2}(\mathbb{R})\right)^{N}
$$

with $X_{0}=\operatorname{span}\left\{\frac{d w}{d y}\right\}$ is invertible and possesses a bounded inverse.

We also introduce the orthogonal projection $\pi_{\varepsilon, \mathbf{t}}^{\perp}: L^{2}(\Omega) \rightarrow \mathcal{C}_{\varepsilon, \mathbf{t}}^{\perp}$ and study the operator $L_{\varepsilon, \mathbf{t}}:=\pi_{\varepsilon, \mathbf{t}}^{\perp} \circ \tilde{L}_{\varepsilon, \mathbf{t}}$, where orthogonality has been defined in $L^{2}(\Omega)$ sense. We will show that $L_{\varepsilon, \mathbf{t}}: \mathcal{K}_{\varepsilon, \mathbf{t}}^{\perp} \rightarrow \mathcal{C}_{\varepsilon, \mathbf{t}}^{\perp}$ is invertible with a bounded inverse provided $\max \left(\frac{\varepsilon}{\sqrt{D}}, D\right)$ is small enough. In proving this, we will use the fact that this system is the limit of the operator $L_{\varepsilon, \mathbf{t}}$ as $\max \left(\frac{\varepsilon}{\sqrt{D}}, D\right) \rightarrow 0$. This statement is contained in the following proposition.

Proposition 9 There exist positive constants $\bar{\delta}, \lambda$ such that for $\max \left(\frac{\varepsilon}{\sqrt{D}}, D\right) \in(0, \bar{\delta})$ and all $\mathbf{t} \in \Omega_{\eta}$, we have

$$
\left\|L_{\varepsilon, \mathbf{t}} \phi_{\varepsilon}\right\|_{L^{2}\left(\Omega_{\varepsilon}\right)} \geqslant \lambda\left\|\phi_{\varepsilon}\right\|_{H^{2}\left(\Omega_{\varepsilon}\right)}
$$

Further, the map

$$
L_{\varepsilon, \mathbf{t}}=\pi_{\varepsilon, \mathbf{t}} \circ \tilde{L}_{\varepsilon, \mathbf{t}}: \mathcal{K}_{\varepsilon, \mathbf{t}}^{\perp} \rightarrow \mathcal{C}_{\varepsilon, \mathbf{t}}^{\perp}
$$

is surjective.

Proof Suppose (6.4) is false. Then, there exist sequences $\left\{\varepsilon_{k}\right\},\left\{D_{k}\right\},\left\{\mathbf{t}^{k}\right\},\left\{\phi^{k}\right\}$ such that $\max \left(\frac{\varepsilon_{k}}{\sqrt{D_{k}}}, D_{k}\right) \rightarrow 0, \mathbf{t}^{k} \in \Omega_{\eta}, \phi^{k}=\phi_{\varepsilon_{k}} \in \mathcal{K}_{\varepsilon_{k}, \mathbf{t}^{k}}^{\perp}, k=1,2, \ldots$ and

$$
\begin{aligned}
& \left\|L_{\epsilon_{k}, \mathbf{t}^{k}} \phi^{k}\right\|_{L^{2}\left(\Omega_{\varepsilon_{k}}\right)} \rightarrow 0 \quad \text { as } k \rightarrow \infty, \\
& \left\|\phi^{k}\right\|_{H^{2}\left(\Omega_{\varepsilon_{k}}\right)}=1, \quad k=1,2, \ldots
\end{aligned}
$$


We define $\phi_{\varepsilon, i}, i=1,2, \ldots, N$ and $\phi_{\varepsilon, N+1}$ as follows:

$$
\begin{gathered}
\phi_{\varepsilon, i}(x)=\phi_{\varepsilon}(x) \chi\left(\frac{x-t_{i}}{\delta_{\varepsilon}}\right), \quad x \in \Omega, \\
\phi_{\varepsilon, N+1}(x)=\phi_{\varepsilon}(x)-\sum_{i=1}^{N} \phi_{\varepsilon, i}(x), \quad x \in \Omega .
\end{gathered}
$$

At first (after rescaling), the functions $\phi_{\varepsilon, i}$ are only defined on $\Omega_{\varepsilon}$. However, by a standard result, they can be extended to $\mathbb{R}$ such that their norm in $H^{2}(\mathbb{R})$ is bounded by a constant independent of $\varepsilon, D$ and $\mathbf{t}$ for $\max \left(\frac{\varepsilon}{\sqrt{D}}, D\right)$ small enough. In the following, we will study this extension. For simplicity of notation, we keep the same notation for the extension. Since for $i=1,2, \ldots, N$, each sequence $\left\{\phi_{i}^{k}\right\}:=\left\{\phi_{\varepsilon_{k}, i}\right\}(k=1,2, \ldots)$ is bounded in $H_{l o c}^{2}(\mathbb{R})$ it has a weak limit in $H_{l o c}^{2}(\mathbb{R})$, and therefore also a strong limit in $L_{l o c}^{2}(\mathbb{R})$ and $L_{l o c}^{\infty}(\mathbb{R})$. Call these limits $\phi_{i}$. Then, passing to the limit in the equation (6.5) in each of the sets $\Omega_{j}=\left\{x \in \Omega: x=t_{j}+\varepsilon y,|y| \leqslant \frac{\delta_{\varepsilon}}{2 \varepsilon}\right\}$ (we refer to Appendix A of [40] for further details), $\phi=\left(\begin{array}{c}\phi_{1} \\ \phi_{2} \\ \vdots \\ \phi_{N}\end{array}\right)$ solves the system $L \phi=0$. By Lemma $20, \phi \in \operatorname{Ker}(L)=X_{0} \oplus \cdots \oplus X_{0}$. Since $\phi_{\varepsilon_{k}} \in K_{\varepsilon_{k}, t_{k}}^{\perp}$ by taking $k \rightarrow \infty$, we get $\phi \in \operatorname{Ker}(L)^{\perp}$. Therefore, $\phi=0$.

By elliptic estimates, we have $\left\|\phi_{\varepsilon_{k}, i}\right\|_{H^{2}(\mathbb{R})} \rightarrow 0$ as $k \rightarrow \infty$ for $i=1,2, \ldots, N$.

Furthermore, $\phi_{\varepsilon_{k}, N+1} \rightarrow \phi_{N+1}$ in $H^{2}(\mathbb{R})$, where $\Phi_{N+1}$ satisfies

$$
\Delta \phi_{N+1}-\phi_{N+1}=0 \quad \text { in } \mathbb{R} \text {. }
$$

Therefore, we conclude $\phi_{N+1}=0$ and $\left\|\phi_{N+1}^{k}\right\|_{H^{2}(\mathbb{R})} \rightarrow 0$ as $k \rightarrow \infty$. This contradicts $\left\|\phi^{k}\right\|_{H^{2}\left(\Omega_{\varepsilon_{k}}\right)}=1$.

To complete the proof of Proposition 9, we just need to show that the conjugate operator to $L_{\varepsilon, \mathbf{t}}$ (denoted by $L_{\varepsilon, \mathbf{t}}^{*}$ ) is injective from $\mathcal{K}_{\varepsilon, \mathbf{t}}^{\perp}$ to $\mathcal{C}_{\varepsilon, \mathbf{t}}^{\perp}$.

The proof for $L_{\varepsilon, \mathbf{t}}^{*}$ follows along the same lines as for $L_{\varepsilon, \mathbf{t}}$ and is omitted.

Now, we are in the position to solve the equation

$$
\pi_{\epsilon, \mathbf{t}}^{\perp} \circ \mathcal{S}_{\epsilon}\left[w_{\varepsilon, \mathbf{t}}+\phi\right]=0 \text {. }
$$

Since $\left.L_{\epsilon, \mathbf{t}}\right|_{K_{\epsilon, \mathbf{t}}^{\perp}}$ is invertible (call the inverse $L_{\epsilon, \mathbf{t}}^{-1}$ ), we can rewrite this as

$$
\phi=-\left(L_{\epsilon, \mathbf{t}}^{-1} \circ \pi_{\epsilon, \mathbf{t}}^{\perp} \circ \mathcal{S}_{\epsilon}\left[w_{\varepsilon, \mathbf{t}}\right]\right)-\left(L_{\epsilon, \mathbf{t}}^{-1} \circ \pi_{\epsilon, \mathbf{t}}^{\perp} \circ \mathcal{N}_{\epsilon, \mathbf{t}}[\phi]\right) \equiv \mathcal{M}_{\epsilon, \mathbf{t}}[\phi],
$$

where

$$
\mathcal{N}_{\varepsilon, \mathbf{t}}[\phi]=\mathcal{S}_{\varepsilon}\left[w_{\varepsilon, \mathbf{t}}+\phi\right]-\mathcal{S}_{\varepsilon}\left[w_{\varepsilon, \mathbf{t}}\right]-\mathcal{S}_{\varepsilon}^{\prime}\left[w_{\varepsilon, \mathbf{t}}\right] \phi
$$

and the operator $\mathcal{M}_{\epsilon, \mathbf{t}}$ is defined by (6.9) for $\phi \in H^{2}\left(\Omega_{\epsilon}\right)$. We are going to show that the 
operator $\mathcal{M}_{\epsilon, \mathbf{t}}$ is a contraction on

$$
B_{\epsilon, \delta} \equiv\left\{\phi \in H^{2}\left(\Omega_{\epsilon}\right):\|\phi\|_{H^{2}\left(\Omega_{\epsilon}\right)}<\delta\right\}
$$

for suitably chosen $\delta$ if $\max \left(\frac{\varepsilon}{\sqrt{D}}, D\right)$ is small enough. By (5.11) and Proposition 9, we have

$$
\begin{aligned}
\left\|\mathcal{M}_{\epsilon, \mathbf{t}}[\phi]\right\|_{H^{2}\left(\Omega_{\epsilon}\right)} & \leqslant \lambda^{-1}\left(\left\|\pi_{\epsilon, \mathbf{t}}^{\perp} \circ \mathcal{N}_{\epsilon, \mathbf{t}}[\phi]\right\|_{L^{2}\left(\Omega_{\varepsilon}\right)}+\left\|\pi_{\epsilon, \mathbf{t}}^{\perp} \circ \mathcal{S}_{\varepsilon}\left[w_{\varepsilon, \mathbf{t}}\right]\right\|_{L^{2}\left(\Omega_{\varepsilon}\right)}\right) \\
& \leqslant \lambda^{-1} C(c(\delta) \delta)\|\phi\|_{H^{2}\left(\Omega_{\epsilon}\right)}+O\left(\frac{\varepsilon}{\sqrt{D}}\right),
\end{aligned}
$$

where $\lambda>0$ is independent of $\delta>0, \varepsilon>0, D>0$ and $c(\delta) \rightarrow 0$ as $\delta \rightarrow 0$. Similarly, we show that

$$
\left\|\mathcal{M}_{\epsilon, \mathbf{t}}[\phi]-\mathcal{M}_{\epsilon, \mathbf{t}}\left[\phi^{\prime}\right]\right\|_{H^{2}\left(\Omega_{\varepsilon}\right)} \leqslant \lambda^{-1} C(c(\delta) \delta)\left\|\phi-\phi^{\prime}\right\|_{H^{2}\left(\Omega_{\varepsilon}\right)},
$$

where $c(\delta) \rightarrow 0$ as $\delta \rightarrow 0$. If we choose

$$
\delta=2 \lambda^{-1}\left\|\pi_{\epsilon, \mathbf{t}}^{\perp} \circ \mathcal{S}_{\varepsilon}\left[w_{\varepsilon, \mathbf{t}}\right]\right\|_{L^{2}\left(\Omega_{\epsilon}\right)},
$$

then for $\max \left(\frac{\epsilon}{\sqrt{D}}, D\right)$ small enough, the operator $\mathcal{M}_{\epsilon, \mathbf{t}}$ is a contraction on $B_{\varepsilon, \delta}$. The existence of a fixed point $\phi_{\epsilon, \mathbf{t}}$ now follows from the standard contraction mapping principle and $\phi_{\epsilon, \mathbf{t}}$ is a solution of (6.9).

We have thus proved

Lemma 10 There exists $\bar{\delta}>0$ such that for every pair of $\epsilon, \mathbf{t}$ with $0<\epsilon<\bar{\delta}$ and $\mathbf{t} \in \Omega_{\eta}$, there exists a unique $\phi_{\epsilon, \mathbf{t}} \in K_{\epsilon, \mathbf{t}}^{\perp}$ satisfying $\mathcal{S}_{\epsilon}\left[w_{\varepsilon, \mathbf{t}}+\phi_{\varepsilon, \mathbf{t}}\right] \in \mathcal{C}_{\varepsilon, \mathbf{t}}$. Furthermore, we have the estimate

$$
\left\|\phi_{\epsilon, \boldsymbol{t}}\right\|_{H^{2}\left(\Omega_{\epsilon}\right)} \leqslant C\left(\frac{\epsilon}{\sqrt{D}}\right) .
$$

Following the same decomposition into leading even and odd terms as discussed in Remark 7 (see also (5.10)) and applying the linear operator $L_{\varepsilon, \mathbf{t}}$ to both of them, we get

$$
\phi_{\varepsilon, \mathbf{t}}=\phi_{\varepsilon, \mathbf{t}, 1}+\phi_{\varepsilon, \mathbf{t}, 2}
$$

where $\phi_{\varepsilon, \mathbf{t}, 1}$ is an even function in the inner variable $y$ which can be estimated as

$$
\left\|\phi_{\varepsilon, \mathbf{t}, 1,}\right\|_{H^{2}\left(\Omega_{\varepsilon}\right)}=O\left(\frac{\varepsilon}{\sqrt{D}}\right)
$$

and $\phi_{\varepsilon, \mathbf{t}, 2}$ is an odd function in the inner variable $y$ which can be estimated as

$$
\left\|\phi_{\varepsilon, \mathbf{t}, 2}\right\|_{H^{2}\left(\Omega_{\varepsilon}\right)}=O\left(\varepsilon \sqrt{D} \log \frac{1}{D}\right) .
$$

Note that the even term is bigger than the odd term but it will drop in many subsequent calculations. 


\section{Existence proof IV: reduced problem}

In this section, we solve the reduced problem. This completes the proof of our main existence result given by Theorem 1 .

By Lemma 10 , for every $\mathbf{t} \in \Omega_{\eta}$, there exists a unique solution $\phi_{\epsilon, \mathbf{t}} \in \mathcal{K}_{\epsilon, \mathbf{t}}^{\perp}$ such that

$$
\mathcal{S}_{\varepsilon}\left[w_{\varepsilon, \mathbf{t}}+\phi_{\varepsilon, \mathbf{t}}\right] \in \mathcal{C}_{\epsilon, \mathbf{t}}
$$

We need to determine $\mathbf{t}^{\varepsilon}=\left(t_{1}^{\varepsilon}, t_{2}^{\varepsilon}, \ldots, t_{N}^{\varepsilon}\right) \in \Omega_{\eta}$ such that

$$
\mathcal{S}_{\varepsilon}\left[w_{\varepsilon, \mathbf{t}^{\varepsilon}}+\phi_{\varepsilon, \mathbf{t}^{\varepsilon}}\right] \perp \mathcal{C}_{\epsilon, \mathbf{t}^{\varepsilon}},
$$

which implies $\mathcal{S}_{\varepsilon}\left[w_{\varepsilon, \mathbf{t}^{\varepsilon}}+\phi_{\varepsilon, \mathbf{t}^{\varepsilon}}\right]=0$.

To this end, let

$$
\begin{gathered}
W_{\epsilon, s}(\mathbf{t}):=\frac{1}{\varepsilon \sqrt{D} \log \frac{1}{D}} \int_{\Omega} \mathcal{S}_{\varepsilon}\left[w_{\varepsilon, \mathbf{t}}+\phi_{\varepsilon, \mathbf{t}}\right] \frac{d \tilde{w}_{s}}{d x} d x, \\
W_{\epsilon}(\mathbf{t}):=\left(W_{\epsilon, 1}(\mathbf{t}), \ldots, W_{\epsilon, N}(\mathbf{t})\right): \Omega_{\eta} \rightarrow \mathbb{R}^{N} .
\end{gathered}
$$

Then, the map $W_{\epsilon}(\mathbf{t})$ is continuous in $\mathbf{t} \in \Omega_{\eta}$ and it remains to find a zero of the vector field $W_{\varepsilon}(\mathbf{t})$.

We compute

$$
\begin{aligned}
& \frac{1}{\varepsilon \sqrt{D} \log \frac{1}{D}} \int_{\Omega} \mathcal{S}_{\varepsilon}\left[w_{\varepsilon, \mathbf{t}}+\phi_{\varepsilon, \mathbf{t}}\right] \frac{d \tilde{w}_{s}}{d x} d x \\
& =\frac{1}{\varepsilon \sqrt{D} \log \frac{1}{D}} \int_{\Omega}\left[\mathcal{S}_{\varepsilon}\left[w_{\varepsilon, \mathbf{t}}\right]+\mathcal{S}_{\varepsilon}^{\prime}\left[w_{\varepsilon, \mathbf{t}}\right]\left(\phi_{\varepsilon, \mathbf{t}}\right)+O\left(\left\|\phi_{\varepsilon, \mathbf{t}}\right\|_{H^{2}(\Omega)}^{2}\right)\right] \frac{d \tilde{w}_{s}}{d x} d x .
\end{aligned}
$$

We first compute the main term given by

$$
\frac{1}{\varepsilon \sqrt{D} \log \frac{1}{D}} \int_{\Omega} \mathcal{S}_{\varepsilon}\left[w_{\varepsilon, \mathbf{t}}\right] \frac{d \tilde{w}_{s}}{d x} d x=c_{s} .
$$

Let $x=t_{s}+\varepsilon y$. By (5.10), we can decompose $\mathcal{S}_{\varepsilon}\left[w_{\varepsilon, \mathrm{t}}\right]$ into odd and even functions. In leading order, only the odd components of $\mathcal{S}_{\varepsilon}\left[w_{\varepsilon, t}\right]$ matter and we have

$$
\frac{1}{\varepsilon \sqrt{D} \log \frac{1}{D}} \int_{\Omega} \mathcal{S}_{\varepsilon}\left[w_{\varepsilon, \mathbf{t}}\right] \frac{d \tilde{w}_{s}}{d x} d x=c_{s, 1}+c_{s, 2},
$$

where

$$
\begin{aligned}
c_{s, 1}= & -\frac{1}{D \log \frac{1}{D}} \int_{\Omega} \frac{x-t_{s}}{\varepsilon} \tilde{w}_{s}^{2} \frac{d w_{s}}{d x} d x \sum_{k \neq s}\left(\mu_{k}\right)^{3 / 2} \hat{\xi}_{k}^{2} e^{-\left|t_{s}-t_{k}\right| / \sqrt{D}}\left(-\frac{t_{s}-t_{k}}{\left|t_{s}-t_{k}\right|}\right) \\
& +O\left(\frac{\varepsilon}{\sqrt{D}}+e^{-2\left(d_{0}-\eta_{0}\right) / \sqrt{D}}\right)
\end{aligned}
$$




$$
\begin{aligned}
= & \frac{1}{D \log \frac{1}{D}} \frac{1}{3} \int_{\mathbb{R}} w_{\mu_{s}}^{3} d y \sum_{k \neq s}\left(\mu_{k}\right)^{3 / 2} \hat{\xi}_{k}^{2} e^{-\left|t_{s}-t_{k}\right| / \sqrt{D}}\left(-\frac{t_{s}-t_{k}}{\left|t_{s}-t_{k}\right|}\right) \\
& +O\left(\frac{\varepsilon}{\sqrt{D}}+e^{-2\left(d_{0}-\eta_{0}\right) / \sqrt{D}}\right) \\
= & \frac{2.4}{D \log \frac{1}{D}}\left(\mu_{s}\right)^{5 / 2} \sum_{k \neq s}\left(\mu_{k}\right)^{3 / 2} \hat{\xi}_{k}^{2} e^{-\left|t_{s}-t_{k}\right| / \sqrt{D}}\left(-\frac{t_{s}-t_{k}}{\left|t_{s}-t_{k}\right|}\right) \\
& +O\left(\frac{\varepsilon}{\sqrt{D}}+e^{-2\left(d_{0}-\eta_{0}\right) / \sqrt{D}}\right) \\
= & \frac{2.4}{D \log \frac{1}{D}}\left(\mu^{0}\right)^{4}\left(\hat{\xi}^{0}\right)^{2} \sum_{k \neq s} e^{-\left|t_{s}-t_{k}\right| / \sqrt{D}}\left(-\frac{t_{s}-t_{k}}{\left|t_{s}-t_{k}\right|}\right)+O\left(\frac{\varepsilon}{\sqrt{D}}+\sqrt{D} \log \frac{1}{D}\right)
\end{aligned}
$$

and

$$
\begin{aligned}
c_{s, 2} & =-\frac{1}{\varepsilon \sqrt{D} \log \frac{1}{D}} \mu^{\prime \prime}\left(t^{0}\right)\left(t_{s}-t^{0}\right) \hat{\xi}_{s} \varepsilon \int_{\Omega} \frac{x-t_{s}}{\varepsilon} \tilde{w}_{s} \frac{d \tilde{w}_{s}}{d x} d x+O\left(\frac{\varepsilon}{\sqrt{D}}+\sqrt{D} \log \frac{1}{D}\right) \\
& =-\frac{1}{\sqrt{D} \log \frac{1}{D}} \mu^{\prime \prime}\left(t^{0}\right)\left(t_{s}-t^{0}\right) \hat{\xi}_{s} \int_{\mathbb{R}} y w_{\mu_{s}} \frac{d w_{\mu_{s}}}{d y} d y+O\left(\frac{\varepsilon}{\sqrt{D}}+\sqrt{D} \log \frac{1}{D}\right) \\
& =\frac{1}{\sqrt{D} \log \frac{1}{D}}\left(\mu_{s}\right)^{3 / 2} \mu^{\prime \prime}\left(t^{0}\right)\left(t_{s}-t^{0}\right) \frac{1}{2} \hat{\xi}_{s} \int_{\mathbb{R}} w^{2} d y+O\left(\frac{\varepsilon}{\sqrt{D}}+\sqrt{D} \log \frac{1}{D}\right) \\
& =\frac{3}{\sqrt{D} \log \frac{1}{D}}\left(\mu^{0}\right)^{3 / 2} \hat{\xi}^{0} \mu^{\prime \prime}\left(t^{0}\right)\left(t_{s}-t^{0}\right)+O\left(\frac{\varepsilon}{\sqrt{D}}+\sqrt{D} \log \frac{1}{D}\right) .
\end{aligned}
$$

In summary, we have

$$
\begin{aligned}
c_{s}= & \frac{2.4}{D \log \frac{1}{D}}\left(\mu^{0}\right)^{4} \sum_{k \neq s}\left(\hat{\xi}^{0}\right)^{2} e^{-\left|t_{s}-t_{k}\right| / \sqrt{D}}\left(-\frac{t_{s}-t_{k}}{\left|t_{s}-t_{k}\right|}\right) \\
& +3 \sqrt{D}\left(\mu^{0}\right)^{3 / 2} \hat{\xi}^{0} \mu^{\prime \prime}\left(t^{0}\right)\left(t_{s}-t^{0}\right)+O\left(\frac{\varepsilon}{\sqrt{D}}+\sqrt{D} \log \frac{1}{D}\right), \quad s=1, \ldots, N .
\end{aligned}
$$

Next, we estimate

$$
\begin{aligned}
\frac{1}{\varepsilon \sqrt{D} \log \frac{1}{D}} \int_{\Omega} \mathcal{S}_{\varepsilon}^{\prime}\left[w_{\varepsilon, \mathbf{t}}\right]\left(\phi_{\varepsilon, \mathbf{t}}\right) \frac{d \tilde{w}_{s}}{d x} d x \\
=\frac{1}{\varepsilon \sqrt{D} \log \frac{1}{D}} \int_{\Omega}\left[\varepsilon^{2} \phi_{\varepsilon, \mathbf{t}}^{\prime \prime}-\mu(x) \phi_{\varepsilon, \mathbf{t}}+\frac{2 w_{\varepsilon, \mathbf{t}}}{T\left[w_{\varepsilon, \mathbf{t}}\right]} \phi_{\varepsilon, \mathbf{t}}-\frac{w_{\varepsilon, \mathbf{t}}^{2}}{\left(T\left[w_{\varepsilon, \mathbf{t}}\right]\right)^{2}}\left(T^{\prime}\left[w_{\varepsilon, \mathbf{t}}\right] \phi_{\varepsilon, \mathbf{t}}\right)\right] \frac{d \tilde{w}_{s}}{d x} d x \\
=\frac{1}{\varepsilon \sqrt{D} \log \frac{1}{D}} \int_{\Omega}\left[\varepsilon^{2} \phi_{\varepsilon, \mathbf{t}}^{\prime \prime}-\mu_{s} \phi_{\varepsilon, \mathbf{t}}+\frac{2 w_{\varepsilon, \mathbf{t}}}{T\left[w_{\varepsilon, \mathbf{t}}\right]} \phi_{\varepsilon, \mathbf{t}}-\frac{w_{\varepsilon, \mathbf{t}}^{2}}{\left(T\left[w_{\varepsilon, \mathbf{t}}\right]\right)^{2}}\left(T^{\prime}\left[w_{\varepsilon, \mathbf{t}}\right] \phi_{\varepsilon, \mathbf{t}}\right)\right] \frac{d \tilde{w}_{s}}{d x} d x \\
\quad+\frac{1}{\varepsilon \sqrt{D} \log \frac{1}{D}} \int_{\Omega}-\left(\mu(x)-\mu\left(t_{s}\right)\right) \phi_{\varepsilon, \mathbf{t}} \frac{d \tilde{w}_{s}}{d x} d x .
\end{aligned}
$$


Integration by parts and and using the derivative of (3.2) gives

$$
\begin{aligned}
\frac{1}{\varepsilon \sqrt{D} \log \frac{1}{D}} \int_{\Omega} \mathcal{S}_{\varepsilon}^{\prime}\left[w_{\varepsilon, \mathbf{t}}\right]\left(\phi_{\varepsilon, \mathbf{t}}\right) \frac{d \tilde{w}_{s}}{d x} d x \\
=\frac{1}{\varepsilon \sqrt{D} \log \frac{1}{D}} \int_{\Omega}\left[\left[\frac{1}{T\left[w_{\varepsilon, \mathbf{t}}\right]}-\frac{1}{\hat{\xi}_{s}}\right] 2 \hat{\xi}_{s} \tilde{w}_{s} \phi_{\varepsilon, \mathbf{t}}-\frac{\left(\hat{\xi}_{s} \tilde{w}_{s}\right)^{2}}{\left(T\left[w_{\varepsilon, \mathbf{t}}\right]\right)^{2}}\left(T^{\prime}\left[w_{\varepsilon, \mathbf{t}}\right] \phi_{\varepsilon, \mathbf{t}}\right)\right] \frac{d \tilde{w}_{s}}{d x} d x \\
\quad+\frac{1}{\varepsilon \sqrt{D} \log \frac{1}{D}} \int_{\Omega}-\left(\mu(x)-\mu\left(t_{s}\right)\right) \phi_{\varepsilon, \mathbf{t}} \frac{d \tilde{w}_{s}}{d x} d x \\
=O\left(\frac{\varepsilon}{\sqrt{D}}\right) .
\end{aligned}
$$

This implies

$$
\begin{aligned}
W_{\varepsilon, s}(\mathbf{t})= & \frac{2.4}{D \log \frac{1}{D}}\left(\mu^{0}\right)^{4} \sum_{k \neq s}\left(\hat{\xi}^{0}\right)^{2} e^{-\left|t_{s}-t_{k}\right| / \sqrt{D}}\left(-\frac{t_{s}-t_{k}}{\left|t_{s}-t_{k}\right|}\right) \\
& +3 \sqrt{D}\left(\mu^{0}\right)^{3 / 2} \hat{\xi}^{0} \mu^{\prime \prime}\left(t^{0}\right)\left(t_{s}-t^{0}\right)+O\left(\frac{\varepsilon}{\sqrt{D}}+\sqrt{D} \log \frac{1}{D}\right), \quad s=1, \ldots, N
\end{aligned}
$$

which follows from (6.11) and (6.12). Now, for given small $\varepsilon>0$, we have to determine $\mathbf{t}^{\varepsilon} \in \Omega_{\eta}$ such that $W_{\varepsilon, s}\left(\mathbf{t}^{\varepsilon}\right)=0$ for $s=1, \ldots, N$.

We first consider the limiting case which only takes into account the leading terms and set

$$
\begin{aligned}
W_{0}(\mathbf{t})= & 2.4 \frac{1}{D \log \frac{1}{D}}\left(\mu^{0}\right)^{4}\left(\hat{\xi}^{0}\right)^{2} \sum_{k,|k-s|=1} e^{-\left|t_{s}-t_{k}\right| / \sqrt{D}}\left(-\frac{t_{s}-t_{k}}{\left|t_{s}-t_{k}\right|}\right) \\
& +3 \sqrt{D}\left(\mu^{0}\right)^{3 / 2} \hat{\xi}^{0} \mu^{\prime \prime}\left(t^{0}\right)\left(t_{s}-t^{0}\right) .
\end{aligned}
$$

We compute $W_{0}\left(\mathbf{t}^{*}\right)=0$, where $\mathbf{t}^{*}$ satisfies

$$
\begin{gathered}
\frac{t_{s}^{*}-t_{s-1}^{*}}{\sqrt{D}}=\log \frac{1}{D}-\log \log \frac{1}{D} \\
-\log \left(\frac{5 \mu^{\prime \prime}\left(t^{0}\right)}{16 \mu^{0}}\right)-\log [(s-1)(N+1-s)]+O\left(\frac{\log \log \frac{1}{D}}{\log \frac{1}{D}}\right), \\
\frac{1}{N} \sum_{k=1}^{N} t_{k}^{*}=t^{0} .
\end{gathered}
$$

By (7.6) and (7.7), we have $\mathbf{t}^{*} \in \Omega_{\eta}$ if $D$ is small enough.

We need to find $\mathbf{t}^{\varepsilon} \in \Omega_{\eta}$ such that $W_{\varepsilon}\left(\mathbf{t}^{\varepsilon}\right)=0$.

Setting $\mathbf{e}=(1,1 \ldots, 1)^{T}$, we have

$$
\frac{c}{\sqrt{D} \log \frac{1}{D}} \leqslant\left\|D W_{0}\left(\mathbf{t}^{*}\right) \mathbf{e}\right\| \leqslant \frac{C}{\sqrt{D} \log \frac{1}{D}}
$$


and

For $\mathbf{t} \in \Omega_{\varepsilon}$, we expand

$$
\frac{c}{\sqrt{D}}\|\mathbf{v}\| \leqslant\left\|D W_{0}\left(\mathbf{t}^{*}\right) \mathbf{v}\right\| \leqslant \frac{C}{\sqrt{D}}\|\mathbf{v}\| \quad \text { if } \mathbf{v} \cdot \mathbf{e}=0 .
$$

$$
\begin{gathered}
W_{\varepsilon}(\mathbf{t})=W_{\varepsilon}(\mathbf{t})-W_{0}(\mathbf{t})+W_{0}(\mathbf{t})-W_{0}\left(\mathbf{t}^{*}\right)+W_{0}\left(\mathbf{t}^{*}\right) \\
=O\left(\frac{\varepsilon}{\sqrt{D}}\right) \text { by }(7.4) \\
+D W_{0}\left(\mathbf{t}^{*}\right) \cdot\left(\mathbf{t}-\mathbf{t}^{*}\right)+R_{0}\left(\mathbf{t}-\mathbf{t}^{*}\right) \\
+W_{0}\left(\mathbf{t}^{*}\right),
\end{gathered}
$$

where $R_{0}(\tau)=D^{2} W_{0}\left(\mathbf{t}^{*}\right)(\tau, \tau)+O\left(|\tau|^{3}\right)$. Decomposing $\tau=\mathbf{v}+\alpha \mathbf{e}$, where $\mathbf{v} \cdot \mathbf{e}=0$, we estimate

$$
\left|R_{0}(\tau)\right| \leqslant \frac{c_{4}}{D}|\mathbf{v}|^{2}+\frac{c_{5}}{\sqrt{D} \log \frac{1}{D}} \alpha|\mathbf{v}|+\frac{c_{6}}{\sqrt{D} \log \frac{1}{D}} \alpha^{2} .
$$

Noting that for $\mathbf{t}^{*}+\tau \in \Omega_{\eta}$, we have $|\mathbf{v}| \leqslant \eta \sqrt{D}$ and $\alpha \leqslant \eta \sqrt{D} \log \frac{1}{D}$, we get

$$
\left|R_{0}(\tau)\right| \leqslant \eta^{2}\left(c_{4}+c_{5} \sqrt{D}+c_{6} \sqrt{D} \log \frac{1}{D}\right) .
$$

This implies

$$
\left|\left(D W_{0}\left(\mathbf{t}^{*}\right)\right)^{-1} R_{0}(\tau) \mathbf{v}\right| \leqslant c_{7} \eta^{2} \sqrt{D}
$$

and

$$
\left|\left(D W_{0}\left(\mathbf{t}^{*}\right)\right)^{-1} R_{0}(\tau) \alpha \mathbf{e}\right| \leqslant c_{7} \eta^{2} \sqrt{D} \log \frac{1}{D} .
$$

Setting $\tau=\mathbf{t}-\mathbf{t}^{*}$, we have to determine $\tau$ such that

$$
-\left(D W_{0}\left(\mathbf{t}^{*}\right)\right)^{-1}\left[W_{\varepsilon}\left(\mathbf{t}^{*}+\tau\right)-W_{0}\left(\mathbf{t}^{*}+\tau\right)+R_{0}(\tau)\right]=\tau
$$

and so $\tau$ must be a fixed point of the mapping

$$
\tau \rightarrow M_{\varepsilon, D}(\tau):=-\left(D W_{0}\left(\mathbf{t}^{*}\right)\right)^{-1}\left[W_{\varepsilon}\left(\mathbf{t}^{*}+\tau\right)-W_{0}\left(\mathbf{t}^{*}+\tau\right)+R_{0}(\tau)\right], \quad B_{1} \rightarrow B_{1},
$$

where $B_{1}=\Omega_{\eta}-\mathbf{t}^{*}$ (pointwise). We estimate

$$
\begin{gathered}
\left\|M_{\varepsilon, D}(\tau)\right\|=\left\|-\left(D W_{0}\left(\mathbf{t}^{*}\right)\right)^{-1}\left[W_{\varepsilon}\left(\mathbf{t}^{*}+\tau\right)-W_{0}\left(\mathbf{t}^{*}+\tau\right)+R_{0}(\tau)\right]\right\| \\
\leqslant C\left(\frac{\varepsilon}{\sqrt{D}} \cdot \sqrt{D} \log \frac{1}{D}+\eta^{2} \sqrt{D} \log \frac{1}{D}\right) \sqrt{D} \log \frac{1}{D} .
\end{gathered}
$$

Using projections, we have

$$
\left\|M_{\varepsilon, D}(\tau) \cdot \mathbf{v}\right\| \leqslant C\left(\frac{\varepsilon}{\sqrt{D}}+\eta^{2}\right) \sqrt{D} \quad \text { if } \mathbf{v} \cdot \mathbf{e}=0
$$

and

$$
\left\|M_{\varepsilon, D}(\tau) \cdot(\alpha \mathbf{e})\right\| \leqslant C\left(\frac{\varepsilon}{\sqrt{D}}+\eta^{2}\right) \sqrt{D} \log \frac{1}{D} .
$$


We now determine when the mapping $M_{\varepsilon, D}$ maps from $B_{1}$ into $B_{1}$ for $\max \left(\frac{\varepsilon}{\sqrt{D}}, D\right)$ small enough. We need to have

$$
C\left(\frac{\varepsilon}{\sqrt{D}}+\eta^{2}\right) \leqslant \eta
$$

Now, (7.8) is satisfied if we choose

$$
\eta=2 C \frac{\varepsilon}{\sqrt{D}}
$$

and we assume

$$
C \eta^{2}=4 C^{3} \frac{\varepsilon^{2}}{D} \leqslant C \frac{\varepsilon}{\sqrt{D}}
$$

Note that (7.10) is satisfied if

$$
\frac{\varepsilon}{\sqrt{D}} \leqslant \frac{1}{4 C^{2}}
$$

which holds if $\frac{\varepsilon}{\sqrt{D}}$ is small enough since $\frac{1}{4 C^{2}}$ is a constant which is independent of $\varepsilon$ and D.

By Brouwer's fixed point theorem, the mapping $M_{\varepsilon, D}$ possesses a fixed point $\tau^{\varepsilon} \in B_{1}$. Then, $\mathbf{t}^{\varepsilon}=\mathbf{t}^{*}+\tau^{\varepsilon} \in \Omega_{\eta}$ is the desired solution which satisfies $W_{\varepsilon}\left(\mathbf{t}^{\varepsilon}\right)=0$.

Thus, we have proved the following proposition.

Proposition 11 For $\max \left(\frac{\epsilon}{\sqrt{D}}, D\right)$ small enough, there exist points $\mathbf{t}^{\epsilon} \in \Omega_{\eta}$ with $\mathbf{t}^{\epsilon} \rightarrow \mathbf{t}^{0}$ such that $W_{\epsilon}\left(\mathbf{t}^{\varepsilon}\right)=0$.

Finally, we complete the proof of Theorem 1.

Proof By Proposition 11, there exists $\mathbf{t}^{\varepsilon} \rightarrow \mathbf{t}^{0}$ such that $W_{\varepsilon}\left(\mathbf{t}^{\varepsilon}\right)=0$. Written differently, we have $\mathcal{S}_{\varepsilon}\left[w_{\varepsilon, \mathbf{t}^{\varepsilon}}+\phi_{\varepsilon, \mathbf{t}^{\varepsilon}}\right]=0$. Let $A_{\varepsilon}=\xi_{\varepsilon}\left(w_{\varepsilon, \mathbf{t}^{\varepsilon}}+\phi_{\varepsilon, \mathbf{t}^{\varepsilon}}\right), H_{\varepsilon}=\xi_{\varepsilon} T\left[w_{\varepsilon, \mathbf{t}^{\varepsilon}}+\phi_{\varepsilon, \mathbf{t}^{\varepsilon}}\right]$. By the Maximum Principle, $A_{\varepsilon}>0, H_{\varepsilon}>0$. Moreover, $\left(A_{\varepsilon}, H_{\varepsilon}\right)$ satisfies all the properties of Theorem 1.

\section{Stability proof I: large eigenvalues}

In this section, we study the large eigenvalues which satisfy $\lambda_{\varepsilon} \rightarrow \lambda_{0} \neq 0$ as $\max \left(\frac{\varepsilon}{\sqrt{D}}, D\right)$ $\rightarrow 0$.

Then, we need to analyse the eigenvalue problem

$$
\tilde{L}_{\varepsilon, \mathbf{t}^{\mathrm{\varepsilon}}} \phi_{\varepsilon}=\varepsilon^{2} \Delta \phi_{\varepsilon}-\mu(x) \phi_{\varepsilon}+\frac{2 A_{\varepsilon} \phi_{\varepsilon}}{T\left[A_{\varepsilon}\right]}-\frac{A_{\varepsilon}^{2}}{\left(T\left[A_{\varepsilon}\right]\right)^{2}}\left(T^{\prime}\left[A_{\varepsilon}\right] \phi_{\varepsilon}\right)=\lambda_{\varepsilon} \phi_{\epsilon}
$$

where $\lambda_{\varepsilon}$ is some complex number, $A_{\varepsilon}=w_{\varepsilon, \mathbf{t}^{\varepsilon}}+\phi_{\varepsilon, \mathbf{t}^{\varepsilon}}$ with $\mathbf{t}^{\varepsilon} \in \Omega_{\eta}$ determined in the previous section,

$$
\phi_{\varepsilon} \in H_{N}^{2}(\Omega)
$$


and for $\phi \in L^{2}(\Omega)$, the function $T^{\prime}[A] \phi$ is defined as the unique solution of

$$
\left\{\begin{array}{l}
D \Delta\left(T^{\prime}[A] \phi\right)-\left(1+\tau \lambda_{\varepsilon}\right)\left(T^{\prime}[A] \phi\right)+2 \xi_{\varepsilon} A \phi=0,-1<x<1 \\
\left(T^{\prime}[A] \phi\right)^{\prime}(-1)=\left(T^{\prime}[A] \phi\right)^{\prime}(1)=0
\end{array}\right.
$$

First, we consider the special case $\tau=0$. Because we study the large eigenvalues, there exists some small $c>0$ such that $\left|\lambda_{\varepsilon}\right| \geqslant c>0$ for $\max \left(\frac{\epsilon}{\sqrt{D}}, D\right)$ small enough. We are looking for a condition under which $\operatorname{Re}\left(\lambda_{\varepsilon}\right) \leqslant c<0$ for all eigenvalues $\lambda_{\varepsilon}$ of (8.1), (8.2) if $\max \left(\frac{\varepsilon}{\sqrt{D}}, D\right)$ is small enough, where $c$ is independent of $\varepsilon$ and $D$. If $\operatorname{Re}\left(\lambda_{\varepsilon}\right) \leqslant-c$, then $\lambda_{\varepsilon}$ is a stable large eigenvalue. Therefore, for the rest of this section, we assume that $\operatorname{Re}\left(\lambda_{\varepsilon}\right) \geqslant-c$ and study the stability properties of such eigenvalues.

We first derive the limiting problem of (8.1), (8.2) as $\max \left(\frac{\varepsilon}{\sqrt{D}}, D\right) \rightarrow 0$ which will be given by a system of NLEPs. Let us assume that

$$
\left\|\phi_{\varepsilon}\right\|_{H^{2}\left(\Omega_{\varepsilon}\right)}=1
$$

We cut off $\phi_{\varepsilon}$ as follows: Introduce

$$
\phi_{\varepsilon, j}(y)=\phi_{\varepsilon}(y) \chi\left(\left|\frac{\varepsilon y}{\delta_{\varepsilon}}\right|\right)
$$

where $y=\left(x-t_{j}\right) / \varepsilon$ for $x \in \Omega$, the cut-off function $\chi$ was introduced in (4.8) and $\delta_{\varepsilon}$ satisfies (4.9).

From (8.1), (8.2), using $\operatorname{Re}\left(\lambda_{\varepsilon}\right) \geqslant-c,\left\|\phi_{\varepsilon, \mathrm{t}^{\mathrm{\varepsilon}}}\right\|_{H^{2}\left(\Omega_{\varepsilon}\right)}=O\left(\frac{\varepsilon}{\sqrt{D}}\right)$ and the exponential decay of $A_{\varepsilon}$, it follows that the last two terms in (8.1) converge to zero in $L^{\infty}\left(\Omega \backslash \cup_{j=1}^{N} \Omega_{j}\right)$, where $\Omega_{j}=\left\{x \in \Omega: x=t_{j}+\varepsilon y,|y| \leqslant \frac{\delta_{\varepsilon}}{2 \varepsilon}\right\}$. In particular, their $L^{\infty}\left(\Omega \backslash \cup_{j=1}^{N} \Omega_{j}\right)$ norm satisfies an estimate of $O\left(\frac{\varepsilon}{\sqrt{D}}\right)$. Then, taking the limit in (8.1), it follows that $\phi_{\varepsilon}=O\left(\frac{\varepsilon}{\sqrt{D}}\right)$ uniformly in $\Omega \backslash \cup_{j=1}^{N} \Omega_{j}$ and so

$$
\phi_{\varepsilon}=\sum_{j=1}^{N} \phi_{\varepsilon, j}+O\left(\frac{\varepsilon}{\sqrt{D}}\right) \quad \text { in } H^{2}\left(\Omega_{\varepsilon}\right)
$$

Next, by a standard procedure, we extend $\phi_{\varepsilon, j}$ to a function defined on $\mathbb{R}$ such that

$$
\left\|\phi_{\varepsilon, j}\right\|_{H^{2}(\mathbb{R})} \leqslant C\left\|\phi_{\varepsilon, j}\right\|_{H^{2}\left(\Omega_{\varepsilon}\right)}, \quad j=1, \ldots, N .
$$

Since $\left\|\phi_{\varepsilon}\right\|_{H^{2}\left(\Omega_{\varepsilon}\right)}=1,\left\|\phi_{\varepsilon, j}\right\|_{H^{2}\left(\Omega_{\varepsilon}\right)} \leqslant C$. By taking a subsequence, we may also assume that $\phi_{\varepsilon, j} \rightarrow \phi_{j}$ as $\max \left(\frac{\varepsilon}{\sqrt{D}}, D\right) \rightarrow 0$ in $H^{1}(\mathbb{R})$ for $j=1, \ldots, N$.

Taking the limit $\max \left(\frac{\varepsilon}{\sqrt{D}}, D\right) \rightarrow 0$ with $\lambda_{\varepsilon} \rightarrow \lambda_{0}$ in $(8.1)$, we get

$$
\Delta_{y} \phi_{i}-\mu \phi_{i}+2 w_{\mu_{i}} \phi_{i}
$$

$$
-2 \lim _{D \rightarrow 0}\left(\sum_{k=1}^{N} \hat{G}_{D}\left(t_{i}^{0}, t_{k}^{0}\right) \int_{\mathbb{R}} \hat{\xi}_{k}^{0} w_{\mu_{k}} \phi_{k} d y\right)\left(\sum_{k=1}^{N} \hat{G}_{D}\left(t_{i}^{0}, t_{k}^{0}\right) \int_{\mathbb{R}}\left(\hat{\xi}_{k}^{0} w_{\mu_{k}}\right)^{2} d y\right)^{-1} w_{\mu_{i}}^{2}=\lambda_{0} \phi_{i}
$$


Using the transformation $\phi(y)=\tilde{\phi}\left(\sqrt{\mu^{0}} y\right), w_{\mu^{0}}(y)=\mu w\left(\sqrt{\mu^{0}} y\right)$, where $\mu^{0}=\mu\left(t^{0}\right)=$ $\lim _{\max \left(\frac{\varepsilon}{\sqrt{D}}, D\right) \rightarrow 0} \mu\left(t_{i}\right)$, then from (3.2), we get

$$
\begin{gathered}
\mu^{0} \Delta_{y} \tilde{\phi}_{i}-\mu^{0} \tilde{\phi}_{i}+2 \mu^{0} w \tilde{\phi}_{i} \\
-2 \mu^{0} \lim _{D \rightarrow 0}\left(\sum_{k=1}^{N} \hat{G}_{D}\left(t_{i}^{0}, t_{k}^{0}\right) \int_{\mathbb{R}} \hat{\xi}_{k}^{0} w \phi_{k} d y\right)\left(\sum_{k=1}^{N} \hat{G}_{D}\left(t_{i}^{0}, t_{k}^{0}\right) \int_{\mathbb{R}}\left(\hat{\xi}_{k}^{0} w\right)^{2} d y\right)^{-1} w^{2}=\lambda_{0} \phi_{i} .
\end{gathered}
$$

Using the relations

$$
\begin{aligned}
& \hat{G}_{D}\left(t_{i}^{0}, t_{j}^{0}\right)=\delta_{i k}+O\left(D \log \frac{1}{D}\right), \\
& \hat{\xi}_{k}^{0}=\frac{1}{\left(\mu^{0}\right)^{3 / 2}}\left[1+O\left(D \log \frac{1}{D}\right)\right]
\end{aligned}
$$

and dropping tilde this implies that

$$
\Delta_{y} \phi_{i}-\phi_{i}+2 w \phi_{i}-\frac{2 \int_{\mathbb{R}} w \phi_{i} d y}{\int_{\mathbb{R}} w^{2} d y} w^{2}=\frac{\lambda_{0}}{\mu^{0}} \phi_{i}, \quad i=1, \ldots, N,
$$

where $\phi_{i} \in H^{2}\left(\mathbb{R}^{N}\right)$.

Then, we have

\section{Theorem 12}

Let $\lambda_{\varepsilon}$ be an eigenvalue of (8.1) and (8.2) such that $\operatorname{Re}\left(\lambda_{\varepsilon}\right)>-c$ for some $c>0$.

(1) Suppose that (for suitable sequences $\max \left(\frac{\varepsilon_{n}}{\sqrt{D_{n}}}, D_{n}\right) \rightarrow 0$ ) we have $\lambda_{\varepsilon_{n}} \rightarrow \lambda_{0} \neq 0$. Then, $\lambda_{0}$ is an eigenvalue of the problem (NLEP) given in (8.6).

(2) Let $\lambda_{0} \neq 0$ with $\operatorname{Re}\left(\lambda_{0}\right)>0$ be an eigenvalue of the problem (NLEP) given in (8.6). Then, for $\max \left(\frac{\varepsilon}{\sqrt{D}}, D\right)$ small enough, there is an eigenvalue $\lambda_{\varepsilon}$ of (8.1) and (8.2) with $\lambda_{\varepsilon} \rightarrow \lambda_{0}$ as $\max \left(\frac{\varepsilon}{\sqrt{D}}, D\right) \rightarrow 0$.

Proof (1) of Theorem 12 follows by asymptotic analysis similar to Section 6 .

To prove (2) of Theorem 12, we follow a compactness argument of Dancer [6]. The main idea of his approach is as follows: Let $\lambda_{0} \neq 0$ be an eigenvalue of problem (8.6) with $\operatorname{Re}\left(\lambda_{0}\right)>0$.

Then, we can rewrite (8.1) as follows:

$$
\phi_{\varepsilon}=-R_{\varepsilon}\left(\lambda_{\varepsilon}\right)\left[\frac{2 A \phi_{\varepsilon}}{T[A]}-\frac{A^{2}}{T[A]} T^{\prime}[A] \phi_{\varepsilon}\right],
$$

where $R_{\varepsilon}\left(\lambda_{\varepsilon}\right)$ is the inverse of $-\Delta+\left(\mu(x)+\lambda_{\varepsilon}\right)$ in $H^{2}(\mathbb{R})$ (which exists if $\operatorname{Re}\left(\lambda_{\varepsilon}\right)>$ $-\min _{x \in \mathbb{R}} \mu(x)$ or $\left.\operatorname{Im}\left(\lambda_{\varepsilon}\right) \neq 0\right)$ and the non-local operators have been defined in (4.21) and (8.3), respectively.

The main property is that $R_{\varepsilon}\left(\lambda_{\varepsilon}\right)$ is a compact operator if $\max \left(\frac{\varepsilon}{\sqrt{D}}, D\right)$ is small enough. The rest of the argument follows in the same way as in [6]. 
We now study the stability of (8.1), (8.2) for large eigenvalues explicitly and prove Theorem 2.

By Lemma 22, for any non-zero eigenvalue $\lambda_{0}$ in (8.6), we have

$$
\operatorname{Re}\left(\lambda_{0}\right) \leqslant c_{0}<0 \quad \text { for some } c_{0}>0 .
$$

Thus, by Theorem $12(1)$, for $\max \left(\frac{\varepsilon}{\sqrt{D}}, D\right)$ small enough, all non-zero large eigenvalues of (8.1), (8.2) have strictly negative real parts. More precisely, all eigenvalues $\lambda_{\varepsilon}$ of $(8.1)$, (8.2), for which $\lambda_{\varepsilon} \rightarrow \lambda_{0} \neq 0$ holds, satisfy $\operatorname{Re}\left(\lambda_{\varepsilon}\right) \leqslant-c<0$.

When studying the case $\tau>0$, we have to deal with NLEPs as in (A 1), for which the coefficient $\gamma$ of the non-local term is a function of $\tau \alpha$. Let $\gamma=\gamma(\tau \alpha)$ be a complex function of $\tau \alpha$. Let us suppose that

$$
\gamma(0) \in \mathbb{R}, \quad|\gamma(\tau \alpha)| \leqslant C \text { for } \operatorname{Re}(\alpha)=\alpha_{R} \geqslant 0, \tau \geqslant 0,
$$

where $C$ is a generic constant which is independent of $\tau$ and $\alpha$. In our case, the following simple example of a function $\gamma(\tau \alpha)$ satisfying (8.8) is relevant:

$$
\gamma(\alpha)=\frac{2}{\sqrt{1+\tau \alpha}}
$$

where $\sqrt{1+\tau \alpha}$ denotes the principal branch of the square root function, compare [35].

Now, we have

Lemma 13 ( [43]) Consider the NLEP

$$
\phi^{\prime \prime}-\phi+2 w \phi-\gamma(\tau \alpha) \frac{\int_{\mathbb{R}} w \phi d y}{\int_{\mathbb{R}} w^{2} d y} w^{2}=\alpha \phi,
$$

where $\gamma(\tau \alpha)$ satisfies (8.8). Then, there is a small number $\tau_{0}>0$ such that for $\tau<\tau_{0}$,

(1) if $\gamma(0)<1$, then there is a positive eigenvalue to (A1);

(2) if $\gamma(0)>1$, then for any non-zero eigenvalue $\alpha$ of (8.9), we have

$$
\operatorname{Re}(\alpha) \leqslant-c_{0}<0
$$

Proof Lemma 13 follows from Theorem 19 by a regular perturbation argument. To make sure that the perturbation argument works, we have to show that if $\alpha_{R} \geqslant-c$ (for some $c>0$ ) and $0 \leqslant \tau<\tau_{0}$ (for some $\tau_{0}>0$ ), where $\alpha=\alpha_{R}+\sqrt{-1} \alpha_{I}$, then $|\alpha| \leqslant C$, where $C$ is a generic constant which is independent of $\tau$. In fact, multiplying (8.9) by the conjugate $\bar{\phi}$ of $\phi$ and integration by parts, we obtain that

$$
\int_{\mathbb{R}}\left(\left|\phi^{\prime}\right|^{2}+|\phi|^{2}-2 w|\phi|^{2}\right) d y=-\alpha \int_{\mathbb{R}}|\phi|^{2} d y-\gamma(\tau \alpha) \frac{\int_{\mathbb{R}} w \phi d y}{\int_{\mathbb{R}} w^{2} d y} \int_{\mathbb{R}} w^{2} \bar{\phi} d y .
$$


From the imaginary part of (8.10), we obtain that

$$
\left|\alpha_{I}\right| \leqslant C_{1}|\gamma(\tau \alpha)|
$$

where $C_{1}$ is a positive constant (independent of $\tau$ ). By assumption (8.8), $|\gamma(\tau \alpha)| \leqslant C$ and so $\left|\alpha_{I}\right| \leqslant C$. Taking the real part of $(8.10)$ and noting that

$$
\text { 1.h.s. of }(8.10) \geqslant C \int_{\mathbb{R}}|\phi|^{2} \quad \text { for some } C \in \mathbb{R} \text {, }
$$

we obtain that $\alpha_{R} \leqslant C_{2}$, where $C_{2}$ is a positive constant (independent of $\tau>0$ ). Therefore, $|\alpha|$ is uniformly bounded and hence a regular perturbation argument gives the desired conclusion.

Remark $14 A$ similar argument as in the previous proof shows that for the original eigenvalue problem (8.1), assuming that $\lambda_{R} \geqslant-c$ (for some $c>0$ ), where $\lambda_{\varepsilon}=\lambda_{\varepsilon, R}+\sqrt{-1} \lambda_{\varepsilon, I}$, $0 \leqslant \tau<\tau_{0}$ (for some $\tau_{0}>0$ ), then $\left|\lambda_{\varepsilon}\right| \leqslant C$, where $C$ is a generic constant which is independent of $\varepsilon, \tau$ for all $0<\varepsilon<\varepsilon_{0}$ ( $\varepsilon_{0}$ chosen small enough) and $0<\tau<\tau_{0}$ ( $\tau_{0}$ chosen small enough). This argument is sketched as follows:

We multiply (8.1) by the conjugate $\bar{\phi}_{\varepsilon}$ of $\phi_{\varepsilon}$ and integration by parts, we obtain that

$$
\int_{\Omega_{\varepsilon}}\left(\left|\phi_{\varepsilon}^{\prime}\right|^{2}+\left|\phi_{\varepsilon}\right|^{2}\right) d y-\int_{\Omega_{\varepsilon}} \frac{2 A_{\varepsilon}\left|\phi_{\varepsilon}\right|^{2}}{T\left[A_{\varepsilon}\right]} d y=-\lambda \int_{\Omega_{\varepsilon}}\left|\phi_{\varepsilon}\right|^{2} d y-\int_{\Omega_{\varepsilon}} \frac{A_{\varepsilon}^{2}}{\left(T\left[A_{\varepsilon}\right]\right)^{2}}\left(T^{\prime}\left[A_{\varepsilon}\right] \phi_{\varepsilon}\right) \bar{\phi}_{\varepsilon} d y .
$$

From the imaginary part of (8.11), we obtain that

$$
\left|\lambda_{\varepsilon, I}\right| \leqslant C_{3}
$$

where $C_{3}$ is a positive constant (independent of $\varepsilon$ and $\tau$ for $\varepsilon, \tau$ small enough). Taking the real part of (8.11) and noting that

$$
\text { l.h.s. of }(8.11) \geqslant C \int_{\Omega}\left|\phi_{\varepsilon}\right|^{2} \quad \text { for some } C \in \mathbb{R} \text {, }
$$

we obtain that $\lambda_{\varepsilon, R} \leqslant C_{4}$, where $C_{4}$ is a positive constant (independent of $\varepsilon, \tau>0$ ). Therefore, $\left|\lambda_{\varepsilon}\right|$ is uniformly bounded in $\varepsilon, \tau$ for all $0<\varepsilon<\varepsilon_{0}$ ( $\varepsilon_{0}$ chosen small enough) and $0<\tau<\tau_{0}$ ( $\tau_{0}$ chosen small enough).

Now, Theorem 12 can be extended to the case $\tau>0$ for eigenvalues such that $\operatorname{Re}\left(\tau \lambda_{\varepsilon}\right) \geqslant-\frac{1}{2}$. Then, by Lemma 13 it follows that for $0 \leqslant \tau<\tau_{0}$ all eigenvalues $\lambda_{\varepsilon}$ of (8.1), (8.2), for which $\lambda_{\varepsilon} \rightarrow \lambda_{0} \neq 0$ holds, satisfy $\operatorname{Re}\left(\lambda_{\varepsilon}\right) \leqslant-c<0$.

For $\tau \geqslant 0$, the large eigenvalues in the limit are determined explicitly by the following result from [47]: 
Lemma 15 Let $\lambda=\sqrt{-1} \lambda_{I}$ be an eigenvalue of the problem

$$
\Delta \phi-\phi+2 w \phi-\frac{2}{\sqrt{1+\tau \lambda}} \frac{\int_{\mathbb{R}} w \phi}{\int_{\mathbb{R}} w^{2}} w^{2}=\lambda \phi, \phi \in H^{1}(\mathbb{R}),
$$

where

$$
\tau \geqslant 0, \lambda \in \mathbb{C}, \lambda=\lambda_{R}+i \lambda_{I}, \lambda_{R} \geqslant 0
$$

and we take the principal branch of $\sqrt{1+\tau \lambda}$. Then, $\lambda$ is a solution of the algebraic equation

$$
\begin{aligned}
\frac{\sqrt{1+\tau \lambda}}{2}-1= & -{ }_{4} F_{3}\left\{\begin{array}{rr}
1,3, & -\frac{1}{2}, \quad 2 ; \\
2+\gamma, 2-\gamma, \frac{5}{2} ;
\end{array}\right\} \\
& +\frac{2 \lambda}{3} b_{1} \frac{\Gamma(1+\gamma) \Gamma\left(\frac{5}{2}\right)}{\Gamma\left(\gamma+\frac{3}{2}\right)}{ }_{3} F_{2}\left\{\begin{array}{r}
2+\gamma,-\frac{3}{2}+\gamma, 1+\gamma ; \\
1 \\
1+2 \gamma, \frac{3}{2}+\gamma ;
\end{array}\right\},
\end{aligned}
$$

where $\gamma=\sqrt{1+\lambda}$ and $b_{1}$ is given by

$$
b_{1}=\frac{9}{24} \frac{(\gamma-1)^{3} \gamma}{(\gamma-3 / 2)(\gamma-1 / 2) 2^{2 \gamma}} \frac{\pi}{\sin (\pi(\gamma-1))} .
$$

Here, for two sequences $a_{1}, a_{2}, \ldots, a_{A}$ and $b_{1}, b_{2}, \ldots, b_{B}$, we let the series

$$
\begin{gathered}
1+\frac{a_{1} a_{2} \cdots a_{A}}{b_{1} b_{2} \cdots b_{B}} \frac{z}{1 !}+\frac{\left(a_{1}+1\right)\left(a_{2}+1\right) \cdots\left(a_{A}+1\right)}{\left(b_{1}+1\right)\left(b_{2}+1\right) \cdots\left(b_{B}+1\right)} \frac{z^{2}}{2 !}+\cdots \\
=:{ }_{A} F_{B}\left\{\begin{array}{c}
a_{1}, a_{2}, \ldots, a_{A} ; \\
z \\
b_{1}, b_{2}, \ldots, b_{B} ;
\end{array}\right\}
\end{gathered}
$$

be the generalized Gauss function or generalized hypergeometric function.

In conclusion, we have finished the study of the large eigenvalues (of order $O(1)$ ) and derived results on their stability properties.

It remains to study the small eigenvalues (of order $o(1)$ ) which will be done in the next section.

\section{Stability proof II: characterization of small eigenvalues}

Now, we study the eigenvalue problem (8.1), (8.2) with respect to small eigenvalues. Namely, we assume that $\lambda_{\varepsilon} \rightarrow 0$ as $\max \left(\frac{\varepsilon}{\sqrt{D}}, D\right) \rightarrow 0$. We will show that that the small eigenvalues are given by

$$
\lambda_{\varepsilon} \sim-2 \frac{\varepsilon^{2}}{\hat{\xi}^{0}} \sigma\left(\mathcal{M}\left(\mathbf{t}^{0}\right)\right)
$$

The matrix $\left(\mathcal{M}\left(\mathbf{t}^{0}\right)\right)$ will be defined in (9.8) and given to leading order in (9.12). Before defining and computing the matrix, we have to make a few preparations. 
Let

$$
\bar{w}_{\varepsilon}=w_{\varepsilon, \mathbf{t}^{\varepsilon}}+\phi_{\varepsilon, \mathbf{t}^{\varepsilon}}, \quad \bar{H}_{\varepsilon}=T\left[w_{\varepsilon, \mathbf{t}^{\varepsilon}}+\phi_{\varepsilon, \mathbf{t}^{\varepsilon}}\right],
$$

where $\mathbf{t}^{\varepsilon}=\left(t_{1}^{\varepsilon}, \ldots, t_{N}^{\varepsilon}\right) \in \Omega_{\eta}$.

After re-scaling, the eigenvalue problem (8.1), (8.2) becomes

$$
\left\{\begin{array}{c}
\varepsilon^{2} \Delta \phi_{\varepsilon}-\mu(x) \phi_{\varepsilon}+2 \frac{\bar{w}_{\varepsilon}}{\bar{H}_{\varepsilon}} \phi_{\epsilon}-\frac{\bar{w}_{\varepsilon}^{2}}{\bar{H}_{\varepsilon}^{2}} \psi_{\varepsilon}=\lambda_{\varepsilon} \phi_{\epsilon} \\
D \Delta \psi_{\varepsilon}-\psi_{\varepsilon}+2 \xi_{\varepsilon} \bar{w}_{\varepsilon} \phi_{\epsilon}=\lambda_{\varepsilon} \tau \psi_{\varepsilon} \\
\phi_{\varepsilon}^{\prime}(-1)=\phi_{\varepsilon}^{\prime}(1)=\psi_{\varepsilon}^{\prime}(-1)=\psi_{\varepsilon}^{\prime}(1)=0 .
\end{array}\right.
$$

Here and in the rest of the proof for small eigenvalues, we set $\tau=0$. Since for small eigenvalues, we have $\tau \lambda_{\varepsilon} \rightarrow 0$ the proof and results extended to the case of a fixed constant $\tau>0$.

Throughout this section, we denote

$$
\mu_{j}=\mu\left(t_{j}^{\varepsilon}\right), \quad \mu_{j}^{\prime}=\mu^{\prime}\left(t_{j}^{\varepsilon}\right), \quad \mu_{j}^{\prime \prime}=\mu^{\prime \prime}\left(t_{j}^{\varepsilon}\right)
$$

By the implicit function theorem, there exists a (locally) unique solution $\hat{\xi}(\mathbf{t})=$ $\left(\hat{\xi}_{1}(\mathbf{t}), \ldots, \hat{\xi}_{N}(\mathbf{t})\right)$ of the equation

$$
\sum_{j=1}^{N} \hat{G}_{D}\left(t_{i}, t_{j}\right) \hat{\xi}_{j}^{2} \mu_{j}^{3 / 2}=\hat{\xi}_{i}, \quad i=1, \ldots, N
$$

Moreover, $\hat{\xi}(\mathbf{t})$ is $C^{1}$ for $\mathbf{t} \in \Omega_{\eta}$. Note that we do not want to consider the solution $\hat{\xi}(\mathbf{t})=0$ since it does not correspond to a strictly positive solution.

We have the estimates

$$
\hat{\xi}\left(\mathbf{t}^{\varepsilon}\right)=O(1), \quad \hat{\xi}_{i}\left(\mathbf{t}^{\varepsilon}\right)-\hat{\xi}_{j}\left(\mathbf{t}^{\varepsilon}\right)=O\left(D\left(\log \frac{1}{D}\right)^{2}\right)
$$

As a preparation, we first compute the derivatives of $\hat{\xi}(\mathbf{t})$.

Now from (9.3), we calculate

$$
\begin{aligned}
\nabla_{t_{j}} \hat{\xi}_{i}= & 2 \sum_{l=1}^{N} \hat{G}_{D}\left(t_{i}, t_{l}\right) \hat{\xi}_{l} \mu_{l}^{3 / 2} \nabla_{t_{j}} \hat{\xi}_{l}+\frac{\partial}{\partial t_{j}}\left(\hat{G}_{D}\left(t_{i}, t_{j}\right)\right) \hat{\xi}_{j}^{2} \mu_{j}^{3 / 2} \\
& +\frac{3}{2} \hat{G}_{D}\left(t_{i}, t_{j}\right) \hat{\xi}_{j}^{2} \mu_{j}^{1 / 2} \mu_{j}^{\prime} \quad \text { for } i \neq j, \\
\nabla_{t_{i}} \hat{\xi}_{i}= & 2 \sum_{l=1}^{N} \hat{G}_{D}\left(t_{i}, t_{l}\right) \hat{\xi}_{l} \mu_{l}^{3 / 2} \nabla_{t_{i}} \hat{\xi}_{l}+\sum_{l=1}^{N} \frac{\partial}{\partial t_{i}}\left(\hat{G}_{D}\left(t_{i}, t_{l}\right)\right) \hat{\xi}_{l}^{2} \mu_{l}^{3 / 2}
\end{aligned}
$$




$$
\begin{aligned}
& +\frac{3}{2} \hat{G}_{D}\left(t_{i}, t_{i}\right) \hat{\xi}_{i}^{2} \mu_{i}^{1 / 2} \mu_{i}^{\prime} \\
= & 2 \sum_{l=1}^{N} \hat{G}_{D}\left(t_{i}, t_{l}\right) \hat{\xi}_{l} \mu_{l}^{3 / 2} \nabla_{t_{i}} \hat{\xi}_{l}+\nabla_{t_{i}} \hat{G}_{D}\left(t_{i}, t_{i}\right) \hat{\xi}_{i}^{2} \mu_{i}^{3 / 2}-\frac{5}{4} \hat{\xi}_{i} \frac{\mu^{\prime}\left(t_{i}\right)}{\mu\left(t_{i}\right)} \\
& +\frac{3}{2} \hat{G}_{D}\left(t_{i}, t_{i}\right) \hat{\xi}_{i}^{2} \mu_{i}^{1 / 2} \mu_{i}^{\prime}+F_{i}(\mathbf{t}) \\
= & 2 \hat{G}_{D}\left(t_{i}, t_{i}\right) \hat{\xi}_{i} \mu_{i}^{3 / 2} \nabla_{t_{i}} \hat{\xi}_{i}-\frac{5}{4} \hat{\xi}_{i} \frac{\mu^{\prime}\left(t_{i}\right)}{\mu\left(t_{i}\right)}+F_{i}(\mathbf{t})+O\left(\sqrt{D} \log \frac{1}{D}\right), \quad i=1, \ldots, N .
\end{aligned}
$$

Here, $F(\mathbf{t})$ is the vector field

$$
F(\mathbf{t})=\left(F_{1}(\mathbf{t}), \ldots, F_{N}(\mathbf{t})\right)
$$

where

$$
F_{i}(\mathbf{t})=\frac{5}{4} \hat{\xi}_{i} \frac{\mu^{\prime}\left(t_{i}\right)}{\mu_{i}}+\sum_{l=1}^{N} \nabla_{t_{i}} \hat{G}_{D}\left(t_{i}, t_{l}\right) \hat{\xi}_{l}^{2} \mu_{l}^{3 / 2}, \quad i=1, \ldots, N
$$

We compute

$$
F_{i}(\mathbf{t})=\frac{5}{4} \hat{\xi}_{i} \frac{\mu^{\prime}\left(t_{i}\right)}{\mu_{i}}+\sum_{l,|l-i|=1} \nabla_{t_{i}} \hat{K}_{D}\left(t_{i}, t_{l}\right) \hat{\xi}_{l}^{2} \mu_{l}^{3 / 2}+O\left(D^{3 / 2}\left(\log \frac{1}{D}\right)^{2}\right), \quad i=1, \ldots, N,
$$

by (3.7), (4.17).

Thus, (9.4) implies that

$$
\nabla_{\mathbf{t}} \hat{\xi}(\mathbf{t})=O\left(\sqrt{D} \log \frac{1}{D}\right)
$$

Set

$$
(\mathcal{M}(\mathbf{t}))_{i, j}=\left(\frac{\partial F_{i}(\mathbf{t})}{\partial t_{j}}\right) .
$$

Comparing with (7.5) and Proposition 11, we have $F\left(\mathbf{t}^{\varepsilon}\right)=O\left(\frac{\varepsilon}{\sqrt{D}}+\sqrt{D} \log \frac{1}{D}\right)$ at $\mathbf{t}^{\varepsilon}=$ $\left(t_{1}^{\varepsilon}, \ldots, t_{N}^{\varepsilon}\right)$. In addition, if $\left.\mathcal{M}\left(\mathbf{t}^{\varepsilon}\right)\right)$ is positive definite, then we will show that all small eigenvalues have negative real part when $0 \leqslant \tau<\tau_{0}$ for some $\tau_{0}>0$.

Next, we compute $\mathcal{M}(\mathbf{t})$ using (9.4).

For $i=j$, we have

$$
\begin{aligned}
& \sum_{l=1}^{N} \nabla_{t_{i}}^{2} \hat{G}_{D}\left(t_{i}, t_{l}\right) \hat{\xi}_{l}^{2} \mu_{l}^{3 / 2} \\
& \quad=\sum_{l=1}^{N} \nabla_{t_{i}}^{2} \hat{K}_{D}\left(t_{i}, t_{l}\right) \hat{\xi}_{l}^{2} \mu_{l}^{3 / 2}\left(1+O\left(e^{-2\left(d_{0}-\eta_{0}\right) \sqrt{D}}\right)\right) \\
& \quad=\left[\nabla_{t_{i}}^{2} \hat{K}_{D}\left(t_{i}, t_{i-1}\right) \hat{\xi}_{i-1}^{2} \mu_{i-1}^{3 / 2}+\nabla_{t_{i}}^{2} \hat{K}_{D}\left(t_{i}, t_{i+1}\right) \hat{\xi}_{i+1}^{2} \mu_{i+1}^{3 / 2}\right]\left(1+O\left(D \log \frac{1}{D}\right)\right) \\
& \quad=\left[\nabla_{t_{i}}^{2} \hat{K}_{D}\left(t_{i}, t_{i-1}\right)\left(\hat{\xi}^{0}\right)^{2}\left(\mu^{0}\right)^{3 / 2}+\nabla_{t_{i}}^{2} \hat{K}_{D}\left(t_{i}, t_{i+1}\right)\left(\hat{\xi}^{0}\right)^{2}\left(\mu^{0}\right)^{3 / 2}\right]\left[1+O\left(D\left(\log \frac{1}{D}\right)^{2}\right)\right] .
\end{aligned}
$$


For $|i-j|=1$, we compute in case $j=i-1$

$$
\begin{aligned}
& \sum_{l=1}^{N} \nabla_{t_{i-1}}\left(\nabla_{t_{i}} \hat{G}_{D}\left(t_{i}, t_{l}\right)\right) \hat{\xi}_{l}^{2} \mu_{l}^{3 / 2} \\
& \quad=\sum_{l=1}^{N} \nabla_{t_{i-1}}\left(\nabla_{t_{i}} \hat{K}_{D}\left(t_{i}, t_{l}\right)\right) \hat{\xi}_{l}^{2} \mu_{l}^{3 / 2}\left[1+O\left(e^{-2\left(d_{0}-\eta_{0}\right) \sqrt{D}}\right)\right] \\
& \quad=\nabla_{t_{i-1}}\left(\nabla_{t_{i}} \hat{K}_{D}\left(t_{i}, t_{i-1}\right)\right) \hat{\xi}_{i-1}^{2} \mu_{i-1}^{3 / 2}\left[1+O\left(D \log \frac{1}{D}\right)\right] \\
& =\nabla_{t_{i-1}}\left(\nabla_{t_{i}} \hat{K}_{D}\left(t_{i}, t_{i-1}\right)\right)\left(\hat{\xi}^{0}\right)^{2}\left(\mu^{0}\right)^{3 / 2}\left[1+O\left(D\left(\log \frac{1}{D}\right)^{2}\right)\right] \\
& \quad=-\nabla_{t_{i}}^{2} \hat{K}_{D}\left(t_{i}, t_{i-1}\right)\left(\hat{\xi}^{0}\right)^{2}\left(\mu^{0}\right)^{3 / 2}\left[1+O\left(D\left(\log \frac{1}{D}\right)^{2}\right)\right]
\end{aligned}
$$

and a similar result holds for $j=i+1$. For $|i-j| \geqslant 2$, we have

$$
\sum_{l=1}^{N} \nabla_{t_{j}}\left(\nabla_{t_{i}} \hat{G}_{D}\left(t_{i}, t_{l}\right)\right) \hat{\xi}_{l}^{2} \mu_{l}^{3 / 2}=O\left(D\left(\log \frac{1}{D}\right)^{2}\right)
$$

This implies

$$
\mathcal{M}\left(\mathbf{t}^{\varepsilon}\right)=\left(m_{i j}\left(\mathbf{t}^{\varepsilon}\right)\right)_{i, j=1}^{N}=\left(m_{i j}\left(\mathbf{t}^{0}\right)\right)_{i, j=1}^{N}\left[1+O\left(D\left(\log \frac{1}{D}\right)^{2}\right)\right]
$$

where

$$
\begin{aligned}
m_{i j}(\mathbf{t})= & \left(\hat{\xi}^{0}\right)^{2}\left(\mu^{0}\right)^{3 / 2}\left[\nabla_{t_{i}}^{2}\left[\hat{K}_{D}\left(t_{i}, t_{i-1}\right)+\hat{K}_{D}\left(t_{i}, t_{i+1}\right)\right] \delta_{i, j}\right. \\
& \left.-\nabla_{t_{i}}^{2} \hat{K}_{D}\left(t_{i}, t_{i-1}\right) \delta_{i, j+1}-\nabla_{t_{i}}^{2} \hat{K}_{D}\left(t_{i}, t_{i+1}\right) \delta_{i, j-1}\right]+\frac{5}{4} \hat{\xi}_{i} \frac{\mu_{i}^{\prime \prime}}{\mu_{i}} \delta_{i, j} \\
& +2 \sum_{j=1}^{N} \nabla_{t_{i}} \hat{K}_{D}\left(t_{i}, t_{i-1}\right) \hat{\xi}_{i-1} \nabla_{t_{j}} \hat{\xi}_{i-1} \mu_{i-1}^{3 / 2}+2 \sum_{j=1}^{N} \nabla_{t_{i}} \hat{K}_{D}\left(t_{i}, t_{i+1}\right) \hat{\xi}_{i+1} \nabla_{t_{j}} \hat{\xi}_{i+1} \mu_{i+1}^{3 / 2} \\
& +\frac{3}{2} \nabla_{t_{i}} \hat{K}_{D}\left(t_{i}, t_{i-1}\right) \hat{\xi}_{i-1}^{2} \mu_{i-1}^{1 / 2} \mu_{i-1}^{\prime} \delta_{i, j+1}+\frac{3}{2} \nabla_{t_{i}} \hat{K}_{D}\left(t_{i}, t_{i+1}\right) \hat{\xi}_{i+1}^{2} \mu_{i+1}^{1 / 2} \mu_{i+1}^{\prime} \delta_{i, j-1} \\
& +\frac{5}{4}\left[\nabla_{t_{i}} \hat{\xi}_{i} \frac{\mu_{i}^{\prime}}{\mu_{i}}-\hat{\xi}_{i} \frac{\left(\mu_{i}^{\prime}\right)^{2}}{\mu_{i}^{2}}\right] \delta_{i, j} .
\end{aligned}
$$

Therefore, using (7.6), (7.7) and the estimate (9.7), we have

$$
\begin{aligned}
m_{i j}\left(\mathbf{t}^{\varepsilon}\right)= & \frac{5}{16}\left(\hat{\xi}^{0}\right)^{2}\left(\mu^{0}\right)^{1 / 2} \mu^{\prime \prime}\left(t_{i}^{\varepsilon}\right) \log \frac{1}{D} \\
& \times\left[-(i-1)(N+1-i) \delta_{j, i-1}-i(N-i) \delta_{j, i+1}+[(i-1)(N+1-i)+i(N-i)] \delta_{i, j}\right]
\end{aligned}
$$




$$
\begin{aligned}
& +\frac{5}{4} \hat{\xi}^{0}\left(\mu^{0}\right)^{-1} \mu^{\prime \prime}\left(t_{i}^{\varepsilon}\right) \delta_{i, j}+O\left(D\left(\log \frac{1}{D}\right)^{2}\right) \\
& =\frac{5}{16}\left(\hat{\xi}^{0}\right)^{2}\left(\mu^{0}\right)^{1 / 2} \mu^{\prime \prime}\left(t^{0}\right) \\
& \quad \times\left[\operatorname { l o g } \frac { 1 } { D } \left[-(i-1)(N+1-i) \delta_{i, j-1}-i(N-i) \delta_{i, j+1}+[(i-1)(N+1-i)\right.\right. \\
& \left.\left.\quad+i(N-i)] \delta_{i, j}\right]+4 \delta_{i, j}\right] \\
& +O\left(\sqrt{D} \log \frac{1}{D}\right) .
\end{aligned}
$$

The matrix $\mathcal{M}\left(\mathbf{t}^{\varepsilon}\right)$ will be the leading-order contribution to the small eigenvalues (compare Lemma 23 and the comments following it). Thus, we study the spectrum of the symmetric $N \times N$-matrix $\mathcal{A}$ defined by

$$
\begin{gathered}
a_{s, s}=(s-1)(N-s+1)+s(N-s), \quad s=1, \ldots, N, \\
a_{s, s+1}=a_{s+1, s}=-s(N-s), \quad s=1, \ldots, N-1, \\
a_{s, t}=0, \quad|s-t|>1 .
\end{gathered}
$$

We will show

Lemma 16 The eigenvalues of the matrix $\mathcal{A}$ are given by

$$
\lambda_{n}=n(n+1), \quad n=0,1, \ldots, N-1 .
$$

The corresponding eigenvectors are computed recursively from (9.17).

The matrix $\mathcal{A}$ has eigenvalue $\lambda_{1}=0$ with eigenvector $v_{1}=\mathbf{e}$. To compute the other eigenvalues and eigenvectors of $\mathcal{A}$, we remark that this problem is equivalent to finding a suitable finite-difference approximation $\tilde{u}$ of the differential equation

$$
h^{2} x(1-x) u^{\prime \prime}+\lambda u=0, \quad u^{\prime}(0)=u^{\prime}(1)=0
$$

in the interval $(0,1)$ for uniform step-size $h=\frac{1}{N}$.

More precisely, we identify

$$
v_{i k}=\tilde{u}\left(x_{k-1 / 2}\right) \text { with } x_{k}=\frac{k}{N} \text { and } x_{k-1 / 2}=\frac{k-1 / 2}{N} \text { for } k=1, \ldots, N,
$$

where in (9.16) we replace $x(1-x) u^{\prime \prime}(x)$ by

$$
\begin{aligned}
\frac{1}{h^{2}} & {\left[x_{k-1}\left(1-x_{k-1}\right) \tilde{u}\left(x_{k-3 / 2}\right)+x_{k}\left(1-x_{k}\right) \tilde{u}\left(x_{k+1 / 2}\right)\right.} \\
& \left.-\left[x_{k}\left(1-x_{k}\right)+x_{k-1}\left(1-x_{k-1}\right)\right] \tilde{u}\left(x_{k-1 / 2}\right)\right] \\
= & (k-1)(N-k+1) \tilde{u}\left(t_{k-3 / 2}\right)+k(N-k) \tilde{u}\left(t_{k+1 / 2}\right) \\
& -[(k-1)(N-k+1)+k(N-k)] \tilde{u}\left(t_{k-1 / 2}\right) .
\end{aligned}
$$


To determine the eigenvectors $v_{i}$, we have to solve this finite-difference problem exactly. We assume that the solutions are given by polynomials of degree $n$ (which will be shown later and $n$ will be specified). Using Taylor expansion around $x=x_{k-1 / 2}$ and the identities

$$
x_{k-1}\left(1-x_{k-1}\right)-x_{k}\left(1-x_{k}\right)=-h\left(1-2 x_{k-1 / 2}\right)
$$

and

$$
x_{k-1}\left(1-x_{k-1}\right)+x_{k}\left(1-x_{k}\right)=2 x_{k-1 / 2}\left(1-x_{k-1 / 2}\right)-\frac{h^{2}}{2},
$$

the finite-difference problem is equivalent to

$$
\begin{aligned}
& \left(2 x(1-x)-\frac{h^{2}}{4}\right) \sum_{l=1}^{[n / 2]} \frac{h^{2 l-2}}{(2 l) !} \tilde{u}^{(2 l)}(x) \\
& +(1-2 x) \sum_{l=1}^{[n / 2]} \frac{h^{2 l-2}}{(2 l-1) !} \tilde{u}^{(2 l-1)}(x)+\lambda_{n} \tilde{u}(x)=0, \quad n=0, \ldots, N-1 .
\end{aligned}
$$

Substituting the ansatz

$$
\tilde{u}(x)=\sum_{k=0}^{n} a_{k} x^{k}
$$

into this equation, considering the coefficient of the power $x^{k}, k=0, \ldots, n$, implies that

$$
\begin{aligned}
& \left(\lambda_{n}-k(k+1)\right) a_{k}+(k+1)^{2} a_{k+1} \\
& +\sum_{l=2}^{[n / 2]+1} 2 \frac{h^{2 l-2}}{(2 l) !} \frac{(k+2 l-1) !}{(k-1) !}\left[\frac{k+l}{k} a_{k+2 l-1}-a_{k+2 l-2}\right] \\
& \quad-\sum_{l=1}^{[n / 2]} \frac{h^{2 l}}{2(2 l) !} \frac{(k+2 l) !}{k !} a_{k+2 l}=0
\end{aligned}
$$

where for $k=0$, we put $(0-1) !=1$ in the second line of $(9.17)$.

For $k=n, n=0,1, \ldots, N-1$, this gives

$$
\left(\lambda_{n}-n(n+1)\right) a_{n}+(n+1)^{2} a_{n+1}=0 .
$$

Thus, if $\lambda_{n}=n(n+1)$, we have $a_{n+1}=0$ and the solution $\tilde{u}(x)$ is indeed a polynomial with degree $n$. After choosing the leading coefficient $a_{n} \neq 0$ arbitrarily, from (9.17), we compute $a_{n-1}, a_{n-2}, \ldots, a_{0}$ recursively in a unique way. Then, we set $v_{n}=\left(\tilde{u}\left(t_{1-1 / 2}\right), \tilde{u}\left(t_{2-1 / 2}\right), \ldots, \tilde{u}\left(t_{N-1 / 2}\right)\right)$.

There are two cases. Case 1. $n<N$ : Then, $v_{n} \neq 0$ since otherwise we would have $\tilde{u} \equiv 0$, in contradiction to the fact that we have chosen $\tilde{u}$ to be a non-trivial eigenfunction with $a_{n} \neq 0$. Thus, $\left(\lambda_{n}, v_{n}\right)$ is an eigenpair for $\mathcal{A}$. The eigenvectors $v_{n}, n=1, \ldots, N$ are linearly independent. From Case 1 , we get $N$ eigenpairs with eigenvalues $\lambda_{n}=n(n+1)$ for $n=0, \ldots, N-1$.

Case 2. $n \geqslant N$ : Then, $v_{n}=0$ although $\tilde{u} \neq 0$. The resulting eigenfunctions for $\mathcal{A}$ are trivial and so in this case there are no new eigenpairs. 
Thus, we have found $N$ eigenpairs with linearly independent eigenvectors.

Remark 17 The eigenvector $v_{0}$ with eigenvalue $\lambda_{0}=0$ corresponds to a rigid translation of all $N$ spikes.

The leading eigenpair for mutual movement of spikes is $\left(\lambda_{1}, v_{1}\right)$.

The eigenvector for $\lambda_{1}=2$ can be computed as follows:

$$
\begin{gathered}
\tilde{u}(x)=1-2 x, 0<x<1, \\
v_{1, k}=\tilde{u}\left(t_{k-1 / 2}\right), k=1, \ldots, N, \\
v_{1, k}=1-\frac{2(k-1 / 2)}{N}=\frac{N-2 k+1}{N} .
\end{gathered}
$$

The components of $v_{1, k}$ are linearly increasing and have odd symmetry around the centre of the spike cluster which corresponds to $k=\frac{N+1}{2}$ or $x=\frac{1}{2}$.

Remark 18 The stability of the small eigenvalues follows from the results in [29] but the eigenvalues have not been determined explicitly.

The technical analysis for the small eigenvalues has been postponed to Appendix B.

\section{Conclusion}

We end this paper with a discussion of our results. We have considered a particular biological reaction-diffusion system with two small diffusivities, the Gierer-Meinhardt system with precursor. We have proved the existence and stability of cluster solutions which have three different length scales: a scale of order $\mathrm{O}(1)$ coming from the precursor inhomogeneity and two small scales which are of the same size as the square roots of the small diffusivities. In particular, the cluster solution can be stable for a suitable choice of parameter values.

Such systems and their solutions play an important role in biological modelling to account for the bridging of length scales, e.g. between genetic, nuclear, intra-cellular, cellular and tissue levels. Our solutions incorporate and combine multiple scales in a robust and stable manner. A particular example of biological multi-scale patterns concerns the pattern formation of head (more precisely, hypostome), tentacles and foot in hydra. Meinhardt's model [17] correctly describes the following experimental observation: With tentacle-specific antibodies, Bode et al. [3] have shown that after head removal tentacle activation first reappears at the very tip of the gastric column. Then, this activation becomes shifted away from the tip to a new location, where the tentacles eventually appear. There are different lengthscales involved for this tentacle pattern: diameter of the gastric column, distance between tentacles and diameter of tentacles.

Let us describe the relation of this paper to [17] in more detail. The model in [17] can be explained in simplified form as follows: It consists of three activator-inhibitor systems, accounting for the formation of head, foot and tentacles, respectively. These subsystems are coupled by a joint source density. Further, there is direct interaction between the 
tentacle and head components to account for suppression of tentacle peaks at the site of head peaks. Altogether, the model is a seven-component reaction-diffusion system.

The main link to the results in this paper is to understand tentacle activation near a maximum of the source density. For this effect, the foot components can be neglected and so we are dealing with a five component system only. It is observed experimentally in [3] and computed numerically in [17] that near a sufficiently high local maximum of the source density tentacle peaks appear. Two different cases are studied: (i) if there is already a peak of head activator at this position, the tentacle peaks will appear at ring-shaped positions with the head activator peak in the centre of the ring, or (ii) if there is no previous head activator peak at this position, a tentacle activator peak will form, followed by a head activator peak which causes the tentacle peak to split into multiple peaks which are finally displaced to positions in ring-shaped positions with the activator peak in its centre.

For this effect to happen, it is assumed that the source density changes very slowly in time and acts on a rather long length scale. This corresponds to the precursor inhomogeneity in our model which is independent of time and has an $O(1)$ length scale. The way the source density enters into the model is set up differently than in our paper resulting in a local maximum in [17] having a similar effect to a local minimum in our paper.

We try to model some of these phenomena in a "minimal model" which consists of only two components corresponding to the two tentacle components in [17] coupled to a time-independent source density acting on an $O(1)$ length scale.

The cluster pattern of spikes located in a sub-interval studied in our paper resembles the ring of tentacle peaks reduced to one dimension. (Work on the two-dimensional case is currently in progress.)

It is interesting to note that our paper is successful in modelling isolated tentacles (without head formation) observed in some experimental situations as discussed in [17].

Comparing the two models in this paper and [17] leads to immediate possible extensions of the spike cluster analysis to models which are biologically more realistic by taking into account the following phenomena: (i) the effect of the head activator-inhibitor system could be added to show that the head activator peak pushes out the tentacle activator peaks; (ii) the tentacle activator peaks split easily due to saturation non-linearities in the tentacle subsystem (whereas in this paper, we do not consider the effect of saturation); (iii) add the foot activator-inhibitor system; (iv) replace the precursor inhomogeneity by a time-dependent source density which interacts with the other subsystems dynamically, e.g. it is enhanced by head activator, suppressed by foot activator, diffuses and possibly has its own predetermined inhomogeneity.

There are links of the model in [17] to other fields in biology such as the periodic spacing of secondary structures around a primary organizing region which is observed in the arrangement of leaves and flower elements in plants around the primary meristem [5].

The molecular basis underlying the model in [17] has recently been confirmed experimentally: After treatment of hydra with Alsterpaullone (which stabilizes $\beta$-catenin and thus increases the source density), it has been found that tentacle formation occurs over the whole body column [4]. Numerical computations have confirmed this behaviour [19]. This is in agreement with the pattern of multiple spikes covering the whole interval computed in our paper (see Figures 4-6). 
Systems of the type considered in this paper are a key to understanding the hierarchy of multi-stage biological processes such as in signalling pathways, where typically first largescale structures appear which induce patterns on successively smaller scales. The precursor can represent previous information from an earlier stage of development leading to the formation of fine structure at the present time. The multi-spike cluster in this paper is a typical small-scale pattern which is established near a pre-existing large-scale precursor inhomogeneity.

\section{Acknowledgements}

This research is supported by NSERC of Canada. MW would like to thank the Department of Mathematics at UBC for its kind hospitality. MW would like to thank the Isaac Newton Institute for Mathematical Sciences for its kind hospitality during the programme "Coupling Geometric PDEs with Physics for Cell Morphology, Motility and Pattern Formation” supported by EPSRC Grant Number EP/K032208/1.

\section{References}

[1] Alberti, G. \& Müller, S. (2001) A new approach to variational problems with multiple scales. Commun. Pure Appl. Math. 54(7), 761-825.

[2] Benson, D. L., Maini, P. K. \& Sherratt, J. A. (1998) Unravelling the turing bifurcation using spatially varying diffusion coefficients. J. Math. Biol. 37(5), 381-417.

[3] Bode, P. M., Awad, T. A., Koizumi, O., Nakashima, Y., Grimmelikhuijzen, C. J. P. \& Bode, H. R. (1988) Development of the two-part pattern during regeneration of the head in hydra. Development 102, 223-235.

[4] Broun, M., Gee, L., Reinhardt, B. \& Bode, H. R. (2005) Formation of the head organizer in hydra involves the canonical Wnt pathway. Development 132, 2907-2916.

[5] Coen, E. S. \& Meyerowitz, E. M. (1991) The war of the whorls: Genetic interactions controlling flower development. Nature 353(6339), 31-37.

[6] Dancer, E. N. (2001) On stability and Hopf bifurcations for chemotaxis systems. Methods Appl. Anal. 8(2), 245-256.

[7] Doelman, A., Gardner, R. A. \& Kaper, T. J. (2001) Large stable pulse solutions in reactiondiffusion equations. Indiana Univ. Math. J. 49(1), 443-507.

[8] Doelman, A., Kaper, T. J. \& van Der Ploeg, H. (2001) Spatially periodic and aperiodic multipulse patterns in the one-dimensional Gierer-Meinhardt equation. Methods Appl. Anal. 8(3), 387-414.

[9] EI, S.-I. \& WEI, J. (2002) Dynamics of metastable localized patterns and its application to the interaction of spike solutions for the Gierer-Meinhardt systems in two spatial dimensions. Japan J. Ind. Appl. Math. 19(2), 181-226.

[10] Floer, A. \& Weinstein, A. (1986) Nonspreading wave packets for the cubic Schrödinger equation with a bounded potential, J. Funct. Anal. 69(3), 397-408.

[11] Gierer, A. \& Meinhardt, H. (1972) A theory of biological pattern formation, Kybernetik (Berlin) 12(1), 30-39.

[12] GuI, C. \& WeI, J. (1999) Multiple interior peak solutions for some singular perturbation problems. J. Differ. Equ. 158(1), 1-27.

[13] Gui, C., WeI, J. \& Winter, M. (2000) Multiple boundary peak solutions for some singularly perturbed Neumann problems. Ann. Inst. H. Poincaré Anal. Non Linéaire 17(1), 47-82.

[14] Herschowitz-Kaufman, M. (1975) Bifurcation analysis of nonlinear reaction-diffusion equations II, steady-state solutions and comparison with numerical simulations. Bull. Math. Biol. 37(6), 589-636. 
[15] Iron, D., Ward, M. J. \& WeI, J. (2001) The stability of spike solutions to the one-dimensional Gierer-Meinhardt model. Physica D. 50(1-2), 25-62.

[16] Meinhardt, H. (1982) Models of Biological Pattern Formation, Academic Press, London.

[17] Meinhardt, H. (1993) A model for pattern-formation of hypostome, tentacles, \& foot in hydra: How to form structures close to each other, how to form them at a distance. Dev. Biol. 157(2), 321-333.

[18] Meinhardt, H. (1998) The Algorithmic Beauty of Sea Shells, 2nd ed. Springer, Berlin, Heidelberg.

[19] Meinhardt, H. (2012) Modeling pattern formation in hydra: A route to understanding essential steps in development. Int. J. Dev. Biol. 56(6-8), 447-462.

[20] MüLlER, S. (1993) Singular perturbations as a selection criterion for periodic minimizing sequences. Calc. Var. Partial Differ. Equ. 1(2), 169-204.

[21] NI, W.-M. \& TAKagi, I. (1991) On the shape of least energy solution to a semilinear Neumann problem. Commun. Pure Appl. Math. 41(7), 819-851.

[22] Ni, W.-M. \& TAKagi, I. (1993) Locating the peaks of least energy solutions to a semilinear Neumann problem. Duke Math. J. 70(2), 247-281.

[23] Nishiura, Y. (1995) Coexistence of infinitely many stable solutions to reaction-diffusion equation in the singular limit. In: C. K. R. T. Jones \& U. Kirchgraber (editors), Dynamics Reported: Expositions in Dynamical Systems, Vol. 3, Springer-Verlag, New York, pp. 25-103.

[24] Nishiura, Y., Teramoto, T., Yuan, X. \& Ueda, K. (2007) Dynamics of traveling pulses in heterogeneous media. Chaos 17(3), 037104.

[25] Он, Y. G. (1988) Existence of semi-classical bound states of nonlinear Schrödinger equations with potentials of the class $(V)_{a}$. Comm. PDE 13(12), 1499-1519.

[26] Он, Y. G. (1990) On positive multi-bump bound states of nonlinear Schrödinger equations under multiple-well potentials. Comm. Math. Phys. 131(2), 223-253.

[27] Page, K., Maini, P. K. \& Monk. N. A. M. (2003) Pattern formation in spatially heterogeneous turing reaction-diffusion models. Phys. D 181(1-2), 80-101.

[28] Page, K., Maini, P. K. \& Monk N. A. M. (2005) Complex pattern formation in reactiondiffusion systems with spatially varying parameters. Phys. D 202(1-2), 95-115.

[29] Sandstede, B. (1998) Stability of multiple-pulse solutions, Trans. Am. Math. Soc. 350(2), 429472.

[30] Takagi, I. (1986) Point-condensation for a reaction-diffusion system. J. Differ. Equ. 61(2), 208-249.

[31] Turing, A. M. (1952) The chemical basis of morphogenesis. Phil. Trans. Roy. Soc. Lond. B 237(641), 37-72.

[32] Ward, M. J., McInerney, D., Houston, P., Gavaghan, D. \& Maini, P. K. (2002) The dynamics and pinning of a spike for a reaction-diffusion system. SIAM J. Appl. Math. 62(4), 1297-1328.

[33] Ward, M. J. \& WeI, J. (2002) Asymmetric spike patterns for the one-dimensional GiererMeinhardt model: Equilibria and stability, Eur. J. Appl. Math. 13(3), 283-320.

[34] Ward, M. J. \& WeI, J. (2003) Hopf bifurcation of spike solutions for the shadow GiererMeinhardt system. Eur. J. Appl. Math. 14(6), 677-711.

[35] Ward, M. J. \& WeI, J. (2003) Hopf bifurcations and oscillatory instabilities of solutions for the one-dimensional Gierer-Meinhardt model. J. Nonlinear Sci. 13(2), 209-264.

[36] WeI, J. (1999) On single interior spike solutions of Gierer-Meinhardt system: Uniqueness and spectrum estimates. Eur. J. Appl. Math. 10(4), 353-378.

[37] Wei, J. \& Winter, M. (1998) Stationary solutions for the Cahn-Hilliard equation. Ann. Inst. H. Poincaré Anal. Non Linéaire 15(4), 459-492.

[38] Wei, J. \& Winter, M. (1999) On the two-dimensional Gierer-Meinhardt system with strong coupling, SIAM J. Math. Anal. 30(6), 1241-1263.

[39] WeI, J. \& Winter, M. (2002) Spikes for the two-dimensional Gierer-Meinhardt system: The strong coupling case. J. Differ. Equ. 178(2), 478-518. 
[40] Wei, J. \& Winter, M. (2001) Spikes for the two-dimensional Gierer-Meinhardt system: The weak coupling case. J. Nonlinear Sci. 6(6), 415-458.

[41] WeI, J. \& Winter, M. (2004) Existence and stability analysis of asymmetric patterns for the Gierer-Meinhardt system, J. Math. Pures Appl. 83(4), 433-476.

[42] WeI, J. \& Winter, M. (2007) Symmetric and asymmetric multiple clusters in a reaction-diffusion system. NoDEA Nonlinear Differ. Equ. Appl. 14(5-6), 787-823.

[43] WeI, J. \& Winter, M. (2007) Existence, classification and stability analysis of multiple-peaked solutions for the Gierer-Meinhardt system in R. Methods Appl. Anal. 14(2), 119-163.

[44] WeI, J. \& Winter, M. (2009) Spikes for the Gierer-Meinhardt system with discontinuous diffusion coefficients. J. Nonlinear Sci. 19(3), 301-339.

[45] Wei, J. \& Winter, M. (2009) On the Gierer-Meinhardt system with precursors. Discrete Contin. Dyn. Syst. 25(1), 363-398.

[46] WeI, J. \& Winter, M. (2014) Stability of cluster solutions in a cooperative consumer chain model. J. Math. Biol. 68(1-2), 1-39.

[47] Wei, J. \& Winter, M. (2014) Mathematical Aspects of Pattern Formation in Biological Systems, Applied Mathematical Sciences, Vol. 189, Springer, London.

[48] YIP, N. K. (2006) Structure of stable solutions of a one-dimensional variational problem. ESAIM Control Optim. Calc. Var. 12(4), 721-751.

[49] Yuan, X., Teramoto, T. \& Nishiura, Y. (2007) Heterogeneity-induced defect bifurcation and pulse dynamics for a three-component reaction-diffusion system. Phys. Rev. E 75(3), 036220.

\section{Appendix A: Preliminary results on non-local eigenvalue problems}

In this appendix, we collect some previous results on NLEPs which will be used throughout the paper.

We first recall the following result:

\section{Theorem 19 Consider the NLEP}

$$
\phi^{\prime \prime}-\phi+2 w \phi-\gamma \frac{\int_{\mathbb{R}} w \phi d y}{\int_{\mathbb{R}} w^{2} d y} w^{2}=\alpha \phi .
$$

(1) (Appendix E of [15].) If $\gamma<1$, then there is a positive eigenvalue to (A1).

(2) (Theorem 1.4 of [36].) If $\gamma>1$, then for any non-zero eigenvalue $\alpha$ of (A1), we have

$$
\operatorname{Re}(\alpha) \leqslant-c<0
$$

(3) If $\gamma \neq 1$ and $\alpha=0$, then

$$
\phi=c_{0} w^{\prime}
$$

for some constant $c_{0}$.

Next, we consider the following system of NLEPs:

$$
L \Phi:=\Phi^{\prime \prime}-\Phi+2 w \Phi-2 \frac{\int_{\mathbb{R}} w \Phi d y}{\int_{\mathbb{R}} w^{2} d y} w^{2},
$$


where

$$
\Phi=\left(\begin{array}{l}
\phi_{1} \\
\phi_{2} \\
\vdots \\
\phi_{N}
\end{array}\right) \in\left(H^{2}(\mathbb{R})\right)^{N}
$$

Set

$$
L_{0} u:=u^{\prime \prime}-u+2 w u
$$

where $u \in H^{2}(\mathbb{R})$.

Then, the conjugate operator of $L$ under the scalar product in $L^{2}(\mathbb{R})$ is given by

$$
L^{*} \Psi=\Psi^{\prime \prime}-\Psi+2 w \Psi-2 \frac{\int_{\mathbb{R}} w^{2} \Psi d y}{\int_{\mathbb{R}} w^{2} d y} w,
$$

where

Then, we have the following result.

$$
\Psi=\left(\begin{array}{l}
\psi_{1} \\
\psi_{2} \\
\vdots \\
\psi_{N}
\end{array}\right) \in\left(H^{2}(\mathbb{R})\right)^{N} .
$$

Lemma 20 ( [43]) We have

$$
\operatorname{Ker}(L)=X_{0} \oplus X_{0} \oplus \cdots \oplus X_{0}
$$

where

$$
X_{0}=\operatorname{span}\left\{w^{\prime}(y)\right\}
$$

and

$$
\operatorname{Ker}\left(L^{*}\right)=X_{0} \oplus X_{0} \oplus \cdots \oplus X_{0}
$$

Proof The system (A 2) is in diagonal form. Suppose

$$
L \Phi=0 .
$$

For $l=1,2, \ldots, N$, the $l$ th equation of system (A 2 ) is given by

$$
\Phi_{l}^{\prime \prime}-\Phi_{l}+2 w \Phi_{l}-2 \frac{\int_{\mathbb{R}} w \Phi_{l} d y}{\int_{\mathbb{R}} w^{2} d y} w^{2}=0 .
$$

By Theorem 19 (3) with $\gamma=2$, we have

$$
\Phi_{l} \in X_{0}, \quad l=1, \ldots, N
$$

and (A 5) follows. 
To prove (A 6), we proceed in a similar way for $L^{*}$. The $l$ th equation of (A 4$)$ is given as follows:

$$
\Psi_{l}^{\prime \prime}-\Psi_{l}+2 w \Psi_{l}-2 \frac{\int_{\mathbb{R}} w^{2} \Psi_{l} d y}{\int_{\mathbb{R}} w^{2} d y} w=0
$$

Multiplying (A 9) by $w$ and integrating, we obtain

$$
\int_{\mathbb{R}} w^{2} \Psi_{l} d y=0
$$

Thus, all the non-local terms vanish and we have

$$
L_{0} \Psi_{l}=0, \quad l=1, \ldots, N
$$

By Theorem 19 (3) with $\gamma=0$, this implies

$$
\Psi_{l} \in X_{0}, \quad l=1, \ldots, N
$$

As a consequence of Lemma 20, we have

Lemma 21 ( [43]) The operator

$$
L:\left(H^{2}(\mathbb{R})\right)^{N} \rightarrow\left(L^{2}(\mathbb{R})\right)^{N}, L \Phi=\Phi^{\prime \prime}-\Phi+2 w \Phi-2 \frac{\int_{\mathbb{R}} w \Phi d y}{\int_{\mathbb{R}} w^{2} d y} w^{2},
$$

is invertible if it is restricted as follows:

$$
L:\left(X_{0} \oplus \cdots \oplus X_{0}\right)^{\perp} \cap\left(H^{2}(\mathbb{R})\right)^{N} \rightarrow\left(X_{0} \oplus \cdots \oplus X_{0}\right)^{\perp} \cap\left(L^{2}(\mathbb{R})\right)^{N} .
$$

Moreover, $L^{-1}$ is bounded.

Proof This result follows from the Fredholm Alternative and Lemma 20.

Finally, we study the eigenvalue problem for $L$ :

$$
L \Phi=\alpha \Phi
$$

We have

Lemma 22 For any non-zero eigenvalue $\alpha$ of $(A 11)$, we have $\operatorname{Re}(\alpha) \leqslant-c<0$.

Proof Let $(\Phi, \alpha)$ satisfy the system (A 11). Suppose $\operatorname{Re}(\alpha) \geqslant 0$ and $\alpha \neq 0$. Then, the $l$ th equation of (A 11) becomes

$$
\Phi_{l}^{\prime \prime}-\Phi_{l}+2 w \Phi_{l}-2 \frac{\int_{\mathbb{R}} w \Phi_{l}}{\int_{\mathbb{R}} w^{2}} w^{2}=\alpha \Phi_{l}
$$


By Theorem 19 (2), we conclude that

$$
\operatorname{Re}(\alpha) \leqslant-c<0
$$

Throughout the paper, let $C, c$ denote generic constants which may change from line to line.

\section{Appendix B: Stability proof III - technical analysis of small eigenvalues}

In this section, we perform a technical analysis of the small eigenvalues and conclude the proof of Theorem 2 .

First, let us define

$$
\tilde{w}_{\varepsilon, j}(x)=\chi\left(\frac{x-t_{j}^{\varepsilon}}{\delta_{\varepsilon}}\right) \bar{w}_{\varepsilon}(x), \quad j=1, \ldots, N,
$$

where $\chi(x)$ is given in (4.8) and $\delta_{\varepsilon}$ satisfies (4.9). We define similar to Section 6

$$
\begin{aligned}
& \mathcal{K}_{\varepsilon, \mathbf{t}^{\varepsilon}}^{\text {new }}:=\operatorname{span}\left\{\varepsilon \tilde{w}_{\varepsilon, j}^{\prime}: j=1, \ldots, N\right\} \subset H^{2}\left(\Omega_{\varepsilon}\right), \\
& \mathcal{C}_{\varepsilon, \mathbf{t}^{\varepsilon}}^{\text {new }}:=\operatorname{span}\left\{\varepsilon \tilde{w}_{\varepsilon, j}^{\prime}: j=1, \ldots, N\right\} \subset L^{2}\left(\Omega_{\varepsilon}\right) .
\end{aligned}
$$

Then, it is easy to see that

$$
\bar{w}_{\varepsilon}(x)=\sum_{j=1}^{N} \tilde{w}_{\varepsilon, j}(x)+O\left(\varepsilon^{10}\right)
$$

Note that $\tilde{w}_{\varepsilon, j}$ satisfies

$$
\varepsilon^{2} \Delta \tilde{w}_{\varepsilon, j}-\mu(x) \tilde{w}_{\varepsilon, j}+\frac{\left(\tilde{w}_{\varepsilon, j}\right)^{2}}{\bar{H}_{\varepsilon}}+O\left(\varepsilon^{10}\right)=0 .
$$

Further, we have $\tilde{w}_{\varepsilon, j}(x)=\hat{\xi}_{j} w_{j}\left(\frac{x-t_{j}^{\varepsilon}}{\varepsilon}\right)+O\left(\frac{\varepsilon}{\sqrt{D}}+D\left(\log \frac{1}{D}\right)^{2}\right)$ in $H^{2}\left(\Omega_{\varepsilon}\right)$, where $w_{j}$ has been defined in(4.6).

Thus, $\tilde{w}_{\varepsilon, j}^{\prime}:=\frac{d \tilde{w}_{\varepsilon, j}}{d x}$ satisfies

$$
\varepsilon^{2} \Delta \tilde{w}_{\varepsilon, j}^{\prime}-\mu(x) \tilde{w}_{\varepsilon, j}^{\prime}+\frac{2 \tilde{w}_{\varepsilon, j}}{\bar{H}_{\varepsilon}} \tilde{w}_{\varepsilon, j}^{\prime}-\frac{\tilde{w}_{\varepsilon, j}^{2}}{\left(\bar{H}_{\varepsilon}\right)^{2}} \bar{H}_{\varepsilon}^{\prime}-\mu^{\prime}(x) \tilde{w}_{\varepsilon, j}+O\left(\varepsilon^{9}\right)=0 .
$$

Let us now decompose

$$
\phi_{\varepsilon}=\varepsilon \sum_{j=1}^{N} a_{j}^{\varepsilon} \tilde{w}_{\varepsilon, j}^{\prime}+\phi_{\varepsilon}^{\perp}
$$

where $a_{j}^{\varepsilon}$ are complex numbers and $\phi_{\varepsilon}^{\perp} \perp \mathcal{K}_{\varepsilon, t^{t}}^{\text {new }}$. Note that the scaling factor $\varepsilon$ has been introduced to ensure that $\phi_{\varepsilon}=O(1)$ in $H^{2}\left(\Omega_{\varepsilon}\right)$.

Suppose that $\left\|\phi_{\varepsilon}\right\|_{H^{2}\left(\Omega_{\varepsilon}\right)}=1$. Then, $\left|a_{j}^{\varepsilon}\right| \leqslant C$. 
The decomposition of $\phi_{\varepsilon}$ given in (B 4) implies

$$
\psi_{\varepsilon}=\varepsilon \sum_{j=1}^{N} a_{j}^{\varepsilon} \psi_{\varepsilon, j}+\psi_{\varepsilon}^{\perp},
$$

where $\psi_{\varepsilon, j}$ satisfies

$$
D \Delta \psi_{\varepsilon, j}-\psi_{\varepsilon, j}+2 \xi_{\varepsilon} \bar{w}_{\varepsilon} \tilde{w}_{\varepsilon, j}^{\prime}=0, \quad \psi_{\varepsilon, j}^{\prime}(-1)=\psi_{\varepsilon, j}^{\prime}(1)=0
$$

and $\psi_{\varepsilon}^{\perp}$ is given by

$$
D \Delta \psi_{\varepsilon}^{\perp}-\psi_{\varepsilon}^{\perp}+2 \xi_{\varepsilon} \bar{w}_{\varepsilon} \phi_{\varepsilon}^{\perp}=0, \quad\left(\psi_{\varepsilon}^{\perp}\right)^{\prime}(-1)=\left(\psi_{\varepsilon}^{\perp}\right)^{\prime}(1)=0 .
$$

Substituting the decompositions of $\phi_{\varepsilon}$ and $\psi_{\varepsilon}$ into (9.2) we have, using (B 3),

$$
\begin{aligned}
& \varepsilon \sum_{j=1}^{N} a_{j}^{\varepsilon}\left(\frac{\left(\tilde{w}_{\varepsilon, j}\right)^{2}}{\bar{H}_{\varepsilon}^{2}} \bar{H}_{\varepsilon}^{\prime}-\frac{\left(\bar{w}_{\varepsilon}\right)^{2}}{\bar{H}_{\varepsilon}^{2}} \psi_{\varepsilon, j}\right)+\varepsilon \sum_{j=1}^{N} a_{j}^{\varepsilon} \mu^{\prime}(x) \tilde{w}_{\varepsilon, j} \\
& \quad+\varepsilon^{2} \Delta \phi_{\varepsilon}^{\perp}-\mu(x) \phi_{\varepsilon}^{\perp}+2 \frac{\bar{w}_{\varepsilon}}{\bar{H}_{\varepsilon}} \phi_{\varepsilon}^{\perp}-\frac{\bar{w}_{\varepsilon}^{2}}{\bar{H}_{\varepsilon}^{2}} \psi_{\varepsilon}^{\perp}-\lambda_{\varepsilon} \phi_{\varepsilon}^{\perp}+O\left(\varepsilon^{9}\right) \\
& =\lambda_{\varepsilon}\left(\varepsilon \sum_{j=1}^{N} a_{j}^{\varepsilon} \tilde{w}_{\varepsilon, j}^{\prime}\right) .
\end{aligned}
$$

We first compute

$$
\begin{aligned}
I_{1} & :=\varepsilon \sum_{j=1}^{N} a_{j}^{\varepsilon}\left(\frac{\left(\tilde{w}_{\varepsilon, j}\right)^{2}}{\bar{H}_{\varepsilon}^{2}} \bar{H}_{\varepsilon}^{\prime}-\frac{\left(\bar{w}_{\varepsilon}\right)^{2}}{\bar{H}_{\varepsilon}^{2}} \psi_{\varepsilon, j}\right) \\
& =\varepsilon \sum_{j=1}^{N} a_{j}^{\varepsilon} \frac{\left(\tilde{w}_{\varepsilon, j}\right)^{2}}{\bar{H}_{\varepsilon}^{2}}\left[-\psi_{\varepsilon, j}+\bar{H}_{\varepsilon}^{\prime}\right]-\varepsilon \sum_{j=1}^{N} a_{j}^{\varepsilon} \psi_{\varepsilon, j} \sum_{k \neq j} \frac{\left(\tilde{w}_{\varepsilon, k}\right)^{2}}{\bar{H}_{\varepsilon}^{2}}+O\left(\varepsilon^{9}\right) \\
& =\varepsilon \sum_{j=1}^{N} a_{j}^{\varepsilon} \frac{\left(\tilde{w}_{\varepsilon, j}\right)^{2}}{\bar{H}_{\varepsilon}^{2}}\left[-\psi_{\varepsilon, j}+\bar{H}_{\varepsilon}^{\prime}\right]-\varepsilon \sum_{j=1}^{N} \sum_{k \neq j} a_{k}^{\varepsilon} \psi_{\varepsilon, k} \frac{\left(\tilde{w}_{\varepsilon, j}\right)^{2}}{\bar{H}_{\varepsilon}^{2}}+O\left(\varepsilon^{9}\right) .
\end{aligned}
$$

We estimate $I_{1}$ as follows

$$
\begin{aligned}
I_{1} & =-\varepsilon \sum_{j=1}^{N} \sum_{k=1}^{N} a_{k}^{\varepsilon} \frac{\left(\tilde{w}_{\varepsilon, j}\right)^{2}}{\bar{H}_{\varepsilon}^{2}}\left[\psi_{\varepsilon, k}-\bar{H}_{\varepsilon}^{\prime} \delta_{j k}\right]+O\left(\varepsilon^{9}\right) \\
& =-\varepsilon \sum_{j=1}^{N} \sum_{|k-j|=1} a_{k}^{\varepsilon} \frac{\left(\tilde{w}_{\varepsilon, j}\right)^{2}}{\bar{H}_{\varepsilon}^{2}} \psi_{\varepsilon, k}+O\left(\varepsilon^{9}\right)+O\left(\varepsilon D^{3 / 2}\left(\log \frac{1}{D}\right)^{2}\right) .
\end{aligned}
$$

Let us also put

$$
\tilde{L}_{\varepsilon} \phi_{\varepsilon}^{\perp}:=\varepsilon^{2} \Delta \phi_{\varepsilon}^{\perp}-\mu(x) \phi_{\varepsilon}^{\perp}+\frac{2 \bar{w}_{\varepsilon}}{\bar{H}_{\varepsilon}} \phi_{\varepsilon}^{\perp}-\frac{\bar{w}_{\varepsilon}^{2}}{\bar{H}_{\varepsilon}^{2}} \psi_{\varepsilon}^{\perp}
$$


and

$$
\mathbf{a}^{\varepsilon}:=\left(a_{1}^{\varepsilon}, \ldots, a_{N}^{\varepsilon}\right)^{T}
$$

Multiplying both sides of (B 8) by $\tilde{w}_{\varepsilon, l}^{\prime}$ and integrating over $(-1,1)$, we obtain, using (3.3),

$$
\begin{aligned}
\text { r.h.s. } & =\varepsilon \lambda_{\varepsilon} \sum_{j=1}^{N} a_{j}^{\varepsilon} \int_{-1}^{1} \tilde{w}_{\varepsilon, j}^{\prime} \tilde{w}_{\varepsilon, l}^{\prime} d x \\
& =\lambda_{\varepsilon} a_{l}^{\varepsilon} \hat{\xi}_{l}^{2} \int_{\mathbb{R}}\left(w_{l}^{\prime}(y)\right)^{2} d y\left(1+O\left(\frac{\varepsilon}{\sqrt{D}}+D\left(\log \frac{1}{D}\right)^{2}\right)\right) \\
& =\lambda_{\varepsilon} a_{l}^{\varepsilon} \hat{\xi}_{l}^{2} \mu_{l}^{5 / 2} \int_{\mathbb{R}}\left(w^{\prime}(z)\right)^{2} d z\left(1+O\left(\frac{\varepsilon}{\sqrt{D}}+D\left(\log \frac{1}{D}\right)^{2}\right)\right)
\end{aligned}
$$

and, using (B 9),

$$
\begin{aligned}
\text { 1.h.s. }= & -\varepsilon \sum_{k=1}^{N} a_{k}^{\varepsilon} \int_{-1}^{1} \frac{\tilde{w}_{\varepsilon, l}^{2}}{\bar{H}_{\varepsilon}^{2}}\left[\psi_{\varepsilon, k}-\bar{H}_{\varepsilon}^{\prime} \delta_{l k}\right] \tilde{w}_{\varepsilon, l}^{\prime} d x \\
& +\varepsilon \sum_{j=1}^{N} a_{j}^{\varepsilon} \int_{-1}^{1} \mu^{\prime} \tilde{w}_{\varepsilon, j} \tilde{w}_{\varepsilon, l}^{\prime} d x \\
& +\int_{-1}^{1} \frac{\tilde{w}_{\varepsilon, l}^{2}}{\bar{H}_{\varepsilon}^{2}}\left(\bar{H}_{\varepsilon}^{\prime} \phi_{\varepsilon}^{\perp}\right) d x \\
& -\int_{-1}^{1} \frac{\tilde{w}_{\varepsilon, l}^{2}}{\bar{H}_{\varepsilon}^{2}}\left(\psi_{\varepsilon}^{\perp} \tilde{w}_{\varepsilon, l}^{\prime}\right) d x+\int_{-1}^{1} \mu^{\prime} \phi_{\varepsilon}^{\perp} \tilde{w}_{\varepsilon, l} d x \\
= & \left(J_{1, l}+J_{2, l}+J_{3, l}+J_{4, l}+J_{5, l}\right),
\end{aligned}
$$

where $J_{i, l}, i=1,2,3,4,5$ are defined by the latest equality.

The following is the key lemma.

\section{Lemma 23 We have}

$$
\begin{aligned}
J_{1, l}= & -\varepsilon^{2}\left(\frac{1}{3} \int_{\mathbb{R}} w^{3} d y\right) \hat{\xi}_{l} \mu_{l}^{5 / 2}\left[-\nabla_{t_{l}^{\varepsilon}}^{2} \hat{K}_{D}\left(t_{l}^{\varepsilon}, t_{l-1}^{\varepsilon}\right) \hat{\xi}_{l-1}^{2} \mu_{l-1}^{3 / 2} a_{l-1}^{\varepsilon}\right. \\
& -\nabla_{t_{l}^{t}}^{2} \hat{K}_{D}\left(t_{l}^{\varepsilon}, t_{l+1}^{\varepsilon}\right) \hat{\xi}_{l+1}^{2} \mu_{l+1}^{3 / 2} a_{l+1}^{\varepsilon} \\
& \left.+\left[\nabla_{t_{l}^{\varepsilon}}^{2} \hat{K}_{D}\left(t_{l}^{\varepsilon}, t_{l+1}^{\varepsilon}\right) \hat{\xi}_{l+1}^{2} \mu_{l+1}^{3 / 2}+\nabla_{t_{l}^{\varepsilon}}^{2} \hat{K}_{D}\left(t_{l}^{\varepsilon}, t_{l-1}^{\varepsilon}\right) \hat{\xi}_{l-1}^{2} \mu_{l-1}^{3 / 2}\right] a_{l}^{\varepsilon}\right] \\
& +O\left(\varepsilon^{2}\left(\frac{\varepsilon}{\sqrt{D}}+D\left(\log \frac{1}{D}\right)^{2}\right)\right),
\end{aligned}
$$




$$
\begin{aligned}
& J_{2, l}=-\varepsilon^{2}\left(\frac{5}{12} \int_{\mathbb{R}} w^{3} d y\right) \hat{\xi}_{l}^{2} \mu_{l}^{3 / 2} \mu_{l}^{\prime \prime} a_{l}^{\varepsilon}+O\left(\varepsilon^{2}\left(\frac{\varepsilon}{\sqrt{D}}+D\left(\log \frac{1}{D}\right)^{2}\right)\right), \\
& J_{3, l}=O\left(\varepsilon^{2}\left(\frac{\varepsilon}{\sqrt{D}}+D\left(\log \frac{1}{D}\right)^{2}\right)\right), \\
& J_{4, l}=O\left(\varepsilon^{2}\left(\frac{\varepsilon}{\sqrt{D}}+D\left(\log \frac{1}{D}\right)^{2}\right)\right) \\
& J_{5, l}=O\left(\varepsilon^{2}\left(\frac{\varepsilon}{\sqrt{D}}+D\left(\log \frac{1}{D}\right)^{2}\right)\right)
\end{aligned}
$$

where $a_{l}^{\varepsilon}$ has been defined in (B 11) and

$$
a_{l}^{0}=\lim _{\varepsilon \rightarrow 0} a_{l}^{\varepsilon}, \quad \mathbf{a}^{0}=\left(a_{1}^{0}, \ldots, a_{N}^{0}\right) .
$$

Proof We prove Theorem 1 by using Lemma 23. We compute

$$
\begin{aligned}
\text { 1.h.s. }= & J_{1, l}+J_{2, l}+O\left(\varepsilon^{2}\left(\frac{\varepsilon}{\sqrt{D}}+D\left(\log \frac{1}{D}\right)^{2}\right)\right) \\
= & -\varepsilon^{2}\left(\frac{1}{3} \int_{\mathbb{R}} w^{3} d y\right) \hat{\xi}_{l} \mu_{l}^{5 / 2}\left[-\nabla_{t_{l}^{\varepsilon}}^{2} \hat{K}_{D}\left(t_{l}^{\varepsilon}, t_{l-1}^{\varepsilon}\right) \hat{\xi}_{l-1}^{2} \mu_{l-1}^{3 / 2} a_{l-1}^{\varepsilon}\right. \\
& -\nabla_{t_{l}^{\varepsilon}}^{2} \hat{K}_{D}\left(t_{l}^{\varepsilon}, t_{l+1}^{\varepsilon}\right) \hat{\xi}_{l+1}^{2} \mu_{l+1}^{3 / 2} a_{l+1}^{\varepsilon} \\
& \left.+\left[\nabla_{t_{l}^{\varepsilon}}^{2} \hat{K}_{D}\left(t_{l}^{\varepsilon}, t_{l+1}^{\varepsilon}\right) \hat{\xi}_{l+1}^{2} \mu_{l+1}^{3 / 2}+\nabla_{t_{l}^{t}}^{2} \hat{K}_{D}\left(t_{l}^{\varepsilon}, t_{l-1}^{\varepsilon}\right) \hat{\xi}_{l-1}^{2} \mu_{l-1}^{3 / 2}\right] a_{l}^{\varepsilon}\right] \\
& -\varepsilon^{2}\left(\frac{5}{12} \int_{\mathbb{R}} w^{3} d y\right) \hat{\xi}_{l}^{2} \mu_{l}^{3 / 2} \mu_{l}^{\prime \prime} a_{l}^{\varepsilon}+O\left(\varepsilon^{2}\left(\frac{\varepsilon}{\sqrt{D}}+D\left(\log \frac{1}{D}\right)^{2}\right)\right) .
\end{aligned}
$$

Comparing with r.h.s. and recalling the computation of $\mathcal{M}\left(t^{0}\right)$ at $(9.12)$, we obtain

$$
\begin{aligned}
& -2.4 \varepsilon^{2} \hat{\xi}^{0}\left(\mu^{0}\right)^{5 / 2} \mathcal{M}\left(\mathbf{t}^{0}\right) \mathbf{a}_{\varepsilon}\left(1+O\left(\frac{\varepsilon}{\sqrt{D}}+\sqrt{D} \log \frac{1}{D}\right)\right) \\
& =\lambda_{\varepsilon}\left(\mu^{0}\right)^{5 / 2}\left(\hat{\xi}^{0}\right)^{2} \mathbf{a}_{\varepsilon} \int_{\mathbb{R}}\left(w^{\prime}(y)\right)^{2} d y\left(1+O\left(\frac{\varepsilon}{\sqrt{D}}+\sqrt{D} \log \frac{1}{D}\right)\right),
\end{aligned}
$$

using (2.3). Equation (B 20) shows that the small eigenvalues $\lambda_{\varepsilon}$ of (9.2) are given by

$$
\lambda_{\varepsilon} \sim-2 \frac{\varepsilon^{2}}{\hat{\xi}^{0}} \sigma\left(\mathcal{M}\left(\mathbf{t}^{0}\right)\right),
$$

using (2.3).

Arguing as in Theorem 12, this shows that if all the eigenvalues of $\mathcal{M}\left(t^{0}\right)$ have positive real part, then the small eigenvalues are stable. On the other hand, if $\mathcal{M}\left(t^{0}\right)$ has an 
eigenvalue with negative real part, then there are eigenfunctions and eigenvalues to make the system unstable.

This proves Theorem 2 .

Next, we prove Lemma 23.

Proof We first study the asymptotic behaviour of $\psi_{\varepsilon, j}$.

Lemma 24 We have

$$
\begin{aligned}
\left(\psi_{\varepsilon, k}-\bar{H}_{\varepsilon}^{\prime} \delta_{k l}\right)\left(t_{l}^{\varepsilon}\right)= & -\nabla_{t_{k}^{\varepsilon}} \hat{K}_{D}\left(t_{l}^{\varepsilon}, t_{k}^{\varepsilon}\right) \hat{\xi}_{k}^{2} \mu_{k}^{3 / 2}\left[\delta_{k, l-1}+\delta_{k, l+1}\right]-\delta_{k l} \sum_{m,|m-l|=1} \nabla_{t_{l}^{\varepsilon}} \hat{K}_{D}\left(t_{l}^{\varepsilon}, t_{m}^{\varepsilon}\right) \hat{\xi}_{m}^{2} \mu_{m}^{3 / 2} \\
& +O\left(\sqrt{D} \log \frac{1}{D}\left(\frac{\varepsilon}{\sqrt{D}}+\left(D \log \frac{1}{D}\right)^{2}\right)\right)
\end{aligned}
$$

Proof Note that for $l \neq k$, we have

$$
\begin{aligned}
\psi_{\varepsilon, k}\left(t_{l}^{\varepsilon}\right) & =2 \xi_{\varepsilon} \int_{-1}^{1} G_{D}\left(t_{l}^{\varepsilon}, z\right) \bar{w}_{\varepsilon} \tilde{w}_{\varepsilon, k}^{\prime} d z \\
& =-\nabla_{t_{k}^{\varepsilon}} \hat{G}_{D}\left(t_{k}^{\varepsilon}, t_{l}^{\varepsilon}\right) \hat{\xi}_{k}^{2} \mu_{k}^{3 / 2}+O\left(\sqrt{D} \log \frac{1}{D}\left(\frac{\varepsilon}{\sqrt{D}}+\left(D \log \frac{1}{D}\right)^{2}\right)\right) \\
& =-\nabla_{t_{k}^{\varepsilon}} \hat{K}_{D}\left(t_{k}^{\varepsilon}, t_{l}^{\varepsilon}\right) \hat{\xi}_{k}^{2} \mu_{k}^{3 / 2}+O\left(\sqrt{D} \log \frac{1}{D}\left(\frac{\varepsilon}{\sqrt{D}}+D\left(\log \frac{1}{D}\right)^{2}\right)\right) .
\end{aligned}
$$

Next, we compute $\left(\psi_{\varepsilon, l}-\bar{H}_{\varepsilon}^{\prime}\right)(x)$ for $x$ near $t_{l}^{\varepsilon}$. We first get

$$
\begin{aligned}
\bar{H}_{\varepsilon}(x)= & \xi_{\varepsilon} \int_{-1}^{1} G_{D}(x, z) \bar{w}_{\varepsilon}^{2} d z \\
= & \xi_{\varepsilon} \int_{-\infty}^{+\infty} K_{D}(|z|) \tilde{w}_{\varepsilon, l}^{2}(x+z) d z-\xi_{\varepsilon} \int_{-1}^{1} H_{D}(x, z) \tilde{w}_{\varepsilon, l}^{2} d z \\
& +\xi_{\varepsilon} \sum_{k \neq l} \int_{-1}^{1} G_{D}(x, z) \tilde{w}_{\varepsilon, k}^{2} d z+O\left(\frac{\varepsilon}{\sqrt{D}}+D\left(\log \frac{1}{D}\right)^{2}\right) .
\end{aligned}
$$

Then, we have

$$
\begin{aligned}
\bar{H}_{\varepsilon}^{\prime}(x)= & \xi_{\varepsilon} \int_{-\infty}^{+\infty} K_{D}(|z|)\left(2 \tilde{w}_{\varepsilon, l}(x+z) \tilde{w}_{\varepsilon, l}^{\prime}(x+z)\right) d z-\xi_{\varepsilon} \int_{-1}^{1} \nabla_{x} H_{D}(x, z) \tilde{w}_{\varepsilon, l}^{2} d z \\
& +\xi_{\varepsilon} \sum_{k \neq l} \int_{-1}^{1} \nabla_{x} G_{D}(x, z) \tilde{w}_{\varepsilon, k}^{2} d z+O\left(\sqrt{D} \log \frac{1}{D}\left(\frac{\varepsilon}{\sqrt{D}}+D\left(\log \frac{1}{D}\right)^{2}\right)\right)
\end{aligned}
$$




$$
\begin{aligned}
= & \nabla_{t_{k}^{\varepsilon}} \hat{K}_{D}\left(t_{k}^{\varepsilon}, t_{l}^{\varepsilon}\right) \hat{\xi}_{k}^{2} \mu_{k}^{3 / 2}+\sum_{k,|k-l|=1} \nabla_{x} \hat{K}_{D}\left(x, t_{k}^{\varepsilon}\right) \hat{\xi}_{k}^{2} \mu_{k}^{3 / 2} \\
& +O\left(\sqrt{D} \log \frac{1}{D}\left(\frac{\varepsilon}{\sqrt{D}}+D\left(\log \frac{1}{D}\right)^{2}\right)\right) .
\end{aligned}
$$

This implies

$$
\begin{aligned}
\bar{H}_{\varepsilon}^{\prime}(x)-\psi_{\varepsilon, l}(x)= & -\xi_{\varepsilon} \int_{-1}^{1} \nabla_{x} H_{D}(x, z) \tilde{w}_{\varepsilon, l}^{2} d z+\xi_{\varepsilon} \sum_{k \neq l} \int_{-1}^{1} \nabla_{x} G_{D}(x, z) \tilde{w}_{\varepsilon, k}^{2} d z \\
& +2 \xi_{\varepsilon} \int_{-1}^{1} H_{D}(x, z) \tilde{w}_{\varepsilon, l} \tilde{w}_{\varepsilon, l}^{\prime} d z \\
& +O\left(\sqrt{D} \log \frac{1}{D}\left(\frac{\varepsilon}{\sqrt{D}}+D\left(\log \frac{1}{D}\right)^{2}\right)\right) \\
= & \sum_{k,|k-l|=1} \nabla_{x} \hat{K}_{D}\left(x, t_{k}^{\varepsilon}\right) \hat{\xi}_{k}^{2} \mu_{k}^{3 / 2} \\
& +O\left(\sqrt{D} \log \frac{1}{D}\left(\frac{\varepsilon}{\sqrt{D}}+D\left(\log \frac{1}{D}\right)^{2}\right)\right) .
\end{aligned}
$$

Therefore, we have,

$$
\begin{aligned}
\bar{H}_{\varepsilon}^{\prime}\left(t_{l}^{\varepsilon}\right)-\psi_{\varepsilon, l}\left(t_{l}^{\varepsilon}\right)= & -\xi_{\varepsilon} \int_{-1}^{1} \nabla_{t_{l}^{\varepsilon}} H_{D}\left(t_{l}^{\varepsilon}, z\right) \tilde{w}_{\varepsilon, l}^{2} d z+\xi_{\varepsilon} \sum_{k \neq l} \int_{-1}^{1} \nabla_{t_{l}^{\varepsilon}} G_{D}\left(t_{l}^{\varepsilon}, z\right) \tilde{w}_{\varepsilon, k}^{2} d z \\
& -\nabla_{t_{l}^{\varepsilon}} H_{D}\left(t_{l}^{\varepsilon}, t_{l}^{\varepsilon}\right) \hat{\xi}_{l}^{2} \mu_{l}^{3 / 2}+O\left(\sqrt{D} \log \frac{1}{D}\left(\frac{\varepsilon}{\sqrt{D}}+D\left(\log \frac{1}{D}\right)^{2}\right)\right) \\
= & \sum_{k=1}^{N} \nabla_{t_{l}^{\varepsilon}} \hat{G}_{D}\left(t_{l}^{\varepsilon}, t_{k}^{\varepsilon}\right) \hat{\xi}_{k}^{2} \mu_{k}^{3 / 2}-\nabla_{t_{l}^{\varepsilon}} H_{D}\left(t_{l}^{\varepsilon}, t_{l}^{\varepsilon}\right) \hat{\xi}_{l}^{2} \mu_{l}^{3 / 2} \\
& +O\left(\sqrt{D} \log \frac{1}{D}\left(\frac{\varepsilon}{\sqrt{D}}+D\left(\log \frac{1}{D}\right)^{2}\right)\right) \\
= & \sum_{k,|k-l|=1} \nabla_{t_{l}^{\varepsilon}} \hat{K}_{D}\left(t_{l}^{\varepsilon}, t_{k}^{\varepsilon}\right) \hat{\xi}_{k}^{2} \mu_{k}^{3 / 2} \\
& +O\left(\sqrt{D} \log \frac{1}{D}\left(\frac{\varepsilon}{\sqrt{D}}+D\left(\log \frac{1}{D}\right)^{2}\right)\right)
\end{aligned}
$$

Combining (B 22) and (B 25), we have shown (B 21).

Similar to the proof of Lemma 24, the following result is derived. 
Lemma 25 We have

$$
\begin{aligned}
& \psi_{\varepsilon, k}\left(t_{l}^{\varepsilon}+\varepsilon y\right)-\psi_{\varepsilon, k}\left(t_{l}^{\varepsilon}\right) \\
& =-\varepsilon y \nabla_{t_{l}^{\varepsilon}} \nabla_{t_{k}^{\varepsilon}} \hat{G}_{D}\left(t_{l}^{\varepsilon}, t_{k}^{\varepsilon}\right) \hat{\xi}_{k}^{2} \mu_{k}^{3 / 2}\left[1+O\left(\frac{\varepsilon}{\sqrt{D}} y\right)+O\left(D\left(\log \frac{1}{D}\right)^{2}\right)\right] \\
& =-\varepsilon y \nabla_{t_{l}^{\varepsilon}} \nabla_{t_{k}^{\varepsilon}} \hat{K}_{D}\left(t_{l}^{\varepsilon}, t_{k}^{\varepsilon}\right) \hat{\xi}_{k}^{2} \mu_{k}^{3 / 2}\left[\delta_{l, k-1}+\delta_{l, k+1}\right]\left[1+O\left(\frac{\varepsilon}{\sqrt{D}} y\right)+O\left(D\left(\log \frac{1}{D}\right)^{2}\right)\right]
\end{aligned}
$$

for $l \neq k$ and

$$
\begin{aligned}
& \left(\psi_{\varepsilon, l}-\bar{H}_{\varepsilon}^{\prime}\right)\left(t_{l}^{\varepsilon}+\varepsilon y\right)-\left(\psi_{\varepsilon, l}-\bar{H}_{\varepsilon}^{\prime}\right)\left(t_{l}^{\varepsilon}\right) \\
& =-\varepsilon y \sum_{m=1}^{N} \nabla_{t_{l}^{\varepsilon}}^{2} \hat{G}_{D}\left(t_{l}^{\varepsilon}, t_{m}^{\varepsilon}\right) \hat{\xi}_{m}^{2} \mu_{m}^{3 / 2}\left[1+O\left(\frac{\varepsilon}{\sqrt{D}} y\right)+O\left(D\left(\log \frac{1}{D}\right)^{2}\right)\right] \\
& =-\varepsilon y \sum_{m,|m-l|=1} \nabla_{t_{l}^{\varepsilon}}^{2} \hat{K}_{D}\left(t_{l}^{\varepsilon}, t_{m}^{\varepsilon}\right) \hat{\xi}_{m}^{2} \mu_{m}^{3 / 2}\left[1+O\left(\frac{\varepsilon}{\sqrt{D}} y\right)+O\left(D\left(\log \frac{1}{D}\right)^{2}\right)\right] .
\end{aligned}
$$

For $J_{1, l}$, we compute

$$
\begin{aligned}
J_{1, l}= & -\varepsilon \sum_{k=1}^{N} a_{k}^{\varepsilon} \int_{-1}^{1} \frac{\tilde{w}_{\varepsilon, l}^{2}}{\bar{H}_{\varepsilon}^{2}}\left[\psi_{\varepsilon, k}-\bar{H}_{\varepsilon}^{\prime} \delta_{l k}\right] \tilde{w}_{\varepsilon, l}^{\prime} d x \\
= & -\varepsilon \sum_{k=1}^{N} a_{k}^{\varepsilon} \int_{-1}^{1} \frac{\tilde{w}_{\varepsilon, l}^{2}}{\bar{H}_{\varepsilon}^{2}}\left[\psi_{\varepsilon, k}\left(t_{l}^{\varepsilon}\right)-\bar{H}_{\varepsilon}^{\prime}\left(t_{l}^{\varepsilon}\right) \delta_{l k}\right] \tilde{w}_{\varepsilon, l}^{\prime} d x \\
& -\varepsilon \sum_{k=1}^{N} a_{k}^{\varepsilon} \int_{-1}^{1} \frac{\tilde{w}_{\varepsilon, l}^{2}}{\bar{H}_{\varepsilon}^{2}}\left(\left[\psi_{\varepsilon, k}(x)-\bar{H}_{\varepsilon}^{\prime}(x) \delta_{l k}\right]-\left[\psi_{\varepsilon, k}\left(t_{l}^{\varepsilon}\right)-\bar{H}_{\varepsilon}^{\prime}\left(t_{l}^{\varepsilon}\right) \delta_{l k}\right]\right) \tilde{w}_{\varepsilon, l}^{\prime} d x . \\
= & J_{6, l}+J_{7, l} .
\end{aligned}
$$

For $J_{6, l}$, we use (B 23) and Lemma 24 to obtain

$$
\begin{aligned}
J_{6, l}= & -\frac{2}{3} \varepsilon \sum_{k=1}^{N} a_{k}^{\varepsilon} \int_{-1}^{1} \frac{\tilde{w}_{\varepsilon, l}^{3}}{\bar{H}_{\varepsilon}^{3}} \bar{H}_{\varepsilon}^{\prime}\left[\psi_{\varepsilon, k}\left(t_{l}^{\varepsilon}\right)-\bar{H}_{\varepsilon}^{\prime}\left(t_{l}^{\varepsilon}\right) \delta_{l k}\right] d x \\
= & -\frac{2}{3} \varepsilon^{2} \sum_{k=1}^{N} a_{k}^{\varepsilon}\left(\int_{\mathbb{R}} w_{l}^{3} d y\right) \bar{H}_{\varepsilon}^{\prime}\left(t_{l}^{\varepsilon}\right)\left[\psi_{\varepsilon, k}\left(t_{l}^{\varepsilon}\right)-\bar{H}_{\varepsilon}^{\prime}\left(t_{l}^{\varepsilon}\right) \delta_{l k}\right] \\
& \times\left[1+O\left(\frac{\varepsilon}{\sqrt{D}}+D\left(\log \frac{1}{D}\right)^{2}\right)\right]
\end{aligned}
$$




$$
\begin{aligned}
& =\varepsilon^{2} \sum_{k=1}^{N} a_{k}^{\varepsilon}\left(\frac{2}{3} \int_{\mathbb{R}} w^{3} d y\right) \mu_{l}^{5 / 2} \\
& \times\left[\nabla_{t_{k}^{\varepsilon}} \hat{G}_{D}\left(t_{l}^{\varepsilon}, t_{k}^{\varepsilon}\right) \hat{\xi}_{k}^{2} \mu_{k}^{3 / 2}-\sum_{k=1}^{N} \nabla_{t_{l}^{\varepsilon}} \hat{G}_{D}\left(t_{l}^{\varepsilon}, t_{k}^{\varepsilon}\right) \hat{\xi}_{k}^{2} \mu_{k}^{3 / 2}\right] \\
& \times\left[\sum_{j=1}^{N} \nabla_{t_{l}^{\varepsilon}} \hat{G}_{D}\left(t_{l}^{\varepsilon}, t_{j}^{\varepsilon}\right) \hat{\xi}_{j}^{2} \mu_{j}^{3 / 2}\right]\left[1+O\left(\frac{\varepsilon}{\sqrt{D}}+D\left(\log \frac{1}{D}\right)^{2}\right)\right] \\
& =\varepsilon^{2} \sum_{k=1}^{N} a_{k}^{\varepsilon}\left(\frac{2}{3} \int_{\mathbb{R}} w^{3} d y\right) \mu_{l}^{5 / 2} \\
& \times\left[\nabla_{t_{k}^{\varepsilon}} \hat{K}_{D}\left(t_{l}^{\varepsilon}, t_{k}^{\varepsilon}\right) \hat{\xi}_{k}^{2} \mu_{k}^{3 / 2}\left[\delta_{l, k-1}+\delta_{l, k+1}\right]-\sum_{k,|k-l|=1} \nabla_{t_{l}^{\varepsilon}} \hat{K}_{D}\left(t_{l}^{\varepsilon}, t_{k}^{\varepsilon}\right) \hat{\xi}_{k}^{2} \mu_{k}^{3 / 2}\right] \\
& \times\left[\sum_{j,|j-l|=1} \nabla_{t_{l}^{\varepsilon}} \hat{K}_{D}\left(t_{l}^{\varepsilon}, t_{j}^{\varepsilon}\right) \hat{\xi}_{j}^{2} \mu_{j}^{3 / 2}\right]+O\left(\varepsilon^{2} D\left(\log \frac{1}{D}\right)^{2}\left(\frac{\varepsilon}{\sqrt{D}}+D\left(\log \frac{1}{D}\right)^{2}\right)\right) \\
& =\varepsilon^{2} \sum_{k=1}^{N} a_{k}^{\varepsilon}\left(\frac{2}{3} \int_{\mathbb{R}} w^{3} d y\right) \mu_{l}^{5 / 2} \\
& \times\left[\nabla_{t_{k}^{\varepsilon}} \hat{K}_{D}\left(t_{l}^{\varepsilon}, t_{k}^{\varepsilon}\right) \hat{\xi}_{k}^{2} \mu_{k}^{3 / 2}\left[\delta_{l, k-1}+\delta_{l, k+1}\right]-\sum_{k,|k-l|=1} \nabla_{t_{l}^{\varepsilon}} \hat{K}_{D}\left(t_{l}^{\varepsilon}, t_{k}^{\varepsilon}\right) \hat{\xi}_{k}^{2} \mu_{k}^{3 / 2}\right] \\
& \times\left[\sum_{j,|j-l|=1} \nabla_{t_{l}^{\varepsilon}} \hat{K}_{D}\left(t_{l}^{\varepsilon}, t_{j}^{\varepsilon}\right) \hat{\xi}_{j}^{2} \mu_{j}^{3 / 2}\right]+O\left(\varepsilon^{2} D\left(\log \frac{1}{D}\right)^{2}\left(\frac{\varepsilon}{\sqrt{D}}+D\left(\log \frac{1}{D}\right)^{2}\right)\right) \\
& =O\left(\varepsilon^{2} D\left(\log \frac{1}{D}\right)^{2}\right) \text {. }
\end{aligned}
$$

Similarly, we compute, using Lemma 25, (9.10) and (9.11),

$$
\begin{aligned}
J_{7, l}= & \varepsilon^{2} \hat{\xi}_{l} \int_{\mathbb{R}}\left(y w_{l}^{2} w_{l}^{\prime}(y)\right) d y \sum_{k=1}^{N}\left(\nabla_{t_{l}^{\varepsilon}} \nabla_{t_{k}^{\varepsilon}} \hat{G}_{D}\left(t_{l}^{\varepsilon}, t_{k}^{\varepsilon}\right) \hat{\xi}_{k}^{2} \mu_{k}^{3 / 2}+\sum_{m=1}^{N} \nabla_{t_{l}^{t}}^{2} \hat{G}_{D}\left(t_{l}^{\varepsilon}, t_{m}^{\varepsilon}\right) \hat{\xi}_{m}^{2} \mu_{m}^{3 / 2} \delta_{k, l}\right) a_{k}^{\varepsilon} \\
& +O\left(\varepsilon^{2} \sqrt{D} \log \frac{1}{D}\left(\frac{\varepsilon}{\sqrt{D}}+D\left(\log \frac{1}{D}\right)^{2}\right)\right) \\
= & -\varepsilon^{2} \hat{\xi}_{l}\left(\frac{1}{3} \int_{\mathbb{R}} w^{3} d y\right)\left[\mu_{l}^{5 / 2} \sum_{k,|k-l|=1} \nabla_{t_{l}^{\varepsilon}} \nabla_{t_{k}^{\varepsilon}} \hat{K}_{D}\left(t_{l}^{\varepsilon}, t_{k}^{\varepsilon}\right) \hat{\xi}_{k}^{2} \mu_{k}^{3 / 2} a_{k}^{\varepsilon}\right. \\
& \left.+\sum_{m,|m-l|=1}^{N} \nabla_{t_{l}^{\varepsilon}}^{2} \hat{K}_{D}\left(t_{l}^{\varepsilon}, t_{m}^{\varepsilon}\right) \hat{\xi}_{m}^{2} \mu_{m}^{3 / 2} a_{l}^{\varepsilon}\right]+O\left(\varepsilon^{2} \sqrt{D} \log \frac{1}{D}\left(\frac{\varepsilon}{\sqrt{D}}+D\left(\log \frac{1}{D}\right)^{2}\right)\right) \\
= & -\varepsilon^{2} \hat{\xi}_{l}\left(\frac{1}{3} \int_{\mathbb{R}} w^{3} d y\right) \mu_{l}^{5 / 2}\left[-\sum_{k,|k-l|=1} \nabla_{t_{l}^{2}}^{2} \hat{K}_{D}\left(t_{l}^{\varepsilon}, t_{k}^{\varepsilon}\right) \hat{\xi}_{k}^{2} \mu_{k}^{3 / 2} a_{k}^{\varepsilon}\right.
\end{aligned}
$$




$$
\begin{aligned}
& \left.+\sum_{k,|k-l|=1}^{N} \nabla_{t_{l}^{\xi}}^{2} \hat{K}_{D}\left(t_{l}^{\varepsilon}, t_{k}^{\varepsilon}\right) \hat{\xi}_{k}^{2} \mu_{k}^{3 / 2} a_{l}^{\varepsilon}\right]+O\left(\varepsilon^{2} \sqrt{D} \log \frac{1}{D}\left(\frac{\varepsilon}{\sqrt{D}}+D\left(\log \frac{1}{D}\right)^{2}\right)\right) \\
= & -\varepsilon^{2} \hat{\xi}_{l}\left(\frac{1}{3} \int_{\mathbb{R}} w^{3} d y\right) \mu_{l}^{5 / 2} \sum_{k,|k-l|=1} \nabla_{t_{l}^{\varepsilon}}^{2} \hat{K}_{D}\left(t_{l}^{\varepsilon}, t_{k}^{\varepsilon}\right) \hat{\xi}_{k}^{2} \mu_{k}^{3 / 2}\left(a_{l}^{\varepsilon}-a_{k}^{\varepsilon}\right) \\
& +O\left(\varepsilon^{2} \sqrt{D} \log \frac{1}{D}\left(\frac{\varepsilon}{\sqrt{D}}+D\left(\log \frac{1}{D}\right)^{2}\right)\right) \\
= & -\varepsilon^{2} \hat{\xi}_{l}\left(\frac{1}{3} \int_{\mathbb{R}} w^{3} d y\right) \mu_{l}^{5 / 2}\left[-\nabla_{t_{l-1}^{\varepsilon}}^{2} \hat{K}_{D}\left(t_{l}^{\varepsilon}, t_{l-1}^{\varepsilon}\right) \hat{\xi}_{l-1}^{2} \mu_{l-1}^{3 / 2} a_{l-1}^{\varepsilon}\right. \\
& -\nabla_{t_{l+1}^{\varepsilon}}^{2} \hat{K}_{D}\left(t_{l}^{\varepsilon}, t_{l+1}^{\varepsilon}\right) \hat{\xi}_{l+1}^{2} \mu_{l+1}^{3 / 2} a_{l+1}^{\varepsilon} \\
& \left.+\left[\nabla_{t_{l}^{t}}^{2} \hat{K}_{D}\left(t_{l}^{\varepsilon}, t_{l+1}^{\varepsilon}\right) \hat{\xi}_{l}^{2} \mu_{l+1}^{3 / 2}+\nabla_{t_{l}^{\varepsilon}}^{2} \hat{K}_{D}\left(t_{l}^{\varepsilon}, t_{l-1}^{\varepsilon}\right) \hat{\xi}_{l}^{2} \mu_{l-1}^{3 / 2}\right] a_{l}^{\varepsilon}\right] \\
& +O\left(\varepsilon^{2} \sqrt{D} \log \frac{1}{D}\left(\frac{\varepsilon}{\sqrt{D}}+D\left(\log \frac{1}{D}\right)^{2}\right)\right) .
\end{aligned}
$$

Combining (B 28) and (B 28), we obtain (B 14).

For $J_{2, l}$, integration by parts gives

$$
\begin{aligned}
J_{2, l} & =\varepsilon \sum_{j=1}^{N} a_{j}^{\varepsilon} \int_{-1}^{1} \mu^{\prime} \tilde{w}_{\varepsilon, j} \tilde{w}_{\varepsilon, l}^{\prime} d x \\
& =-\frac{\varepsilon a_{l}^{\varepsilon}}{2} \int_{-1}^{1} \mu^{\prime \prime} \tilde{w}_{\varepsilon, l}^{2} d x\left[1+O\left(\frac{\varepsilon}{\sqrt{D}}+D\left(\log \frac{1}{D}\right)^{2}\right)\right] \\
& =-\frac{\varepsilon^{2} a_{l}^{\varepsilon}}{2} \hat{\xi}_{l}^{2} \mu_{l}^{3 / 2} \mu_{l}^{\prime \prime} \int_{\mathbb{R}} w^{2} d y\left[1+O\left(\frac{\varepsilon}{\sqrt{D}}+D\left(\log \frac{1}{D}\right)^{2}\right)\right]
\end{aligned}
$$

and (B 15) follows.

These are the main terms. The remaining terms are small and we will show that they are of the order $O\left(\varepsilon^{2}\left(\frac{\varepsilon}{\sqrt{D}}+D\left(\log \frac{1}{D}\right)^{2}\right)\right)$.

Similar to the proof of Proposition 9, it follows that $\tilde{L}_{\varepsilon}$ is invertible from $\left(\mathcal{K}_{\varepsilon}^{\text {new }}\right)^{\perp}$ to $\left(\mathcal{C}_{\varepsilon}^{\text {new }}\right)^{\perp}$ with uniformly bounded inverse for $\max \left(\frac{\varepsilon}{\sqrt{D}}, D\right)$ small enough. By (B 8), (B 9), Lemma 24 and the fact that $\tilde{L}_{\varepsilon}$ is uniformly invertible, we deduce that

$$
\left\|\phi_{\varepsilon}^{\perp}\right\|_{H^{2}\left(\Omega_{\varepsilon}\right)}=O\left(\varepsilon \sqrt{D} \log \frac{1}{D}\right)
$$

Then, we have by the equation for $\psi_{\varepsilon}^{\perp}$

$$
\psi_{\varepsilon}^{\perp}\left(t_{j}^{\varepsilon}\right)=2 \xi_{\varepsilon} \int_{-1}^{1} G_{D}\left(t_{j}^{\varepsilon}, z\right) \bar{w}_{\varepsilon} \phi_{\varepsilon}^{\perp} d z=O\left(\varepsilon \sqrt{D} \log \frac{1}{D}\right)
$$


Further, we estimate

$$
\begin{aligned}
\psi_{\varepsilon}^{\perp}\left(t_{j}^{\varepsilon}+\varepsilon y\right)-\psi_{\varepsilon}^{\perp}\left(t_{j}^{\varepsilon}\right) & =2 \xi_{\varepsilon} \int_{-1}^{1}\left[G_{D}\left(t_{j}^{\varepsilon}+\varepsilon y, z\right)-G_{D}\left(t_{j}^{\varepsilon}+\varepsilon y, z\right)\right] \bar{w}_{\varepsilon} \phi_{\varepsilon}^{\perp} d z \\
& =2 \varepsilon y \xi_{\varepsilon} \int_{-1}^{1} \nabla_{t_{j}^{\varepsilon}} G_{D}\left(t_{j}^{\varepsilon} y, z\right) \bar{w}_{\varepsilon} \phi_{\varepsilon}^{\perp} d z\left[1+O\left(\frac{\varepsilon}{\sqrt{D}}+D\left(\log \frac{1}{D}\right)^{2}\right)\right] \\
& =O\left(\varepsilon \sqrt{D} \log \frac{1}{D} \varepsilon \sqrt{D} \log \frac{1}{D} y\right)=O\left(\varepsilon^{2} D\left(\log \frac{1}{D}\right)^{2} y\right)
\end{aligned}
$$

These estimates of $\psi_{\varepsilon}^{\perp}$ and $\phi_{\varepsilon}^{\perp}$ are important for the rest of the proof.

For $J_{3, l}$, we have by (B 23), (B 29)

$$
\begin{aligned}
J_{3, l} & =\bar{H}_{\varepsilon}^{\prime}\left(t_{l}^{\varepsilon}\right) \int_{-1}^{1} \tilde{w}_{\varepsilon, l}^{2} \phi_{\varepsilon}^{\perp} d x\left[1+O\left(\frac{\varepsilon}{\sqrt{D}}+D\left(\log \frac{1}{D}\right)^{2}\right)\right] \\
& =O\left(\sqrt{D} \log \frac{1}{D} \varepsilon\left\|\phi_{\varepsilon}^{\perp}\right\|_{H^{2}\left(\Omega_{\varepsilon}\right)}\right)=O\left(\varepsilon^{2} D\left(\log \frac{1}{D}\right)^{2}\right)
\end{aligned}
$$

which proves (B 16).

For $J_{4, l}$, we decompose

$$
J_{4, l}=J_{8, l}+J_{9, l}
$$

where

$$
\begin{gathered}
J_{8, l}=-\int_{-1}^{1} \frac{\tilde{w}_{\varepsilon, l}^{2}}{\bar{H}_{\varepsilon}^{2}}\left(\psi_{\varepsilon}^{\perp}\left(t_{l}^{\varepsilon}\right) \tilde{w}_{\varepsilon, l}^{\prime}\right) d x, \\
J_{9, l}=-\int_{-1}^{1} \frac{\tilde{w}_{\varepsilon, l}^{2}}{\bar{H}_{\varepsilon}^{2}}\left(\psi_{\varepsilon}^{\perp}(x)-\psi_{\varepsilon}^{\perp}\left(t_{l}^{\varepsilon}\right)\right) \tilde{w}_{\varepsilon, l}^{\prime} d x .
\end{gathered}
$$

For $J_{8, l}$, we have used (B 23), (B 30)

$$
\begin{aligned}
J_{8, l} & =-\psi_{\varepsilon}^{\perp}\left(t_{l}^{\varepsilon}\right) \int_{-1}^{1} \frac{\tilde{w}_{\varepsilon, l}^{2}}{\bar{H}_{\varepsilon}^{2}} \tilde{w}_{\varepsilon, l}^{\prime} d x \\
& =\frac{2}{3} \psi_{\varepsilon}^{\perp}\left(t_{l}^{\varepsilon}\right) \int_{-1}^{1} \frac{\tilde{w}_{\varepsilon, l}^{3}}{\bar{H}_{\varepsilon}^{3}} \bar{H}_{\varepsilon}^{\prime} d x\left[1+O\left(\frac{\varepsilon}{\sqrt{D}}+D\left(\log \frac{1}{D}\right)^{2}\right)\right] \\
& =-\varepsilon \frac{2}{3} \bar{H}_{\varepsilon}^{\prime}\left(t_{l}^{\varepsilon}\right) \psi_{\varepsilon}^{\perp}\left(t_{l}^{\varepsilon}\right) \mu_{l}^{5 / 2}\left(\int_{\mathbb{R}} w^{3} d y\right)\left[1+O\left(\frac{\varepsilon}{\sqrt{D}}+D\left(\log \frac{1}{D}\right)^{2}\right)\right] \\
& =O\left(\varepsilon \sqrt{D} \log \frac{1}{D} \varepsilon \sqrt{D} \log \frac{1}{D}\right)=O\left(\varepsilon^{2} D\left(\log \frac{1}{D}\right)^{2}\right) .
\end{aligned}
$$

For $J_{9, l}$, we have used (B 30)

$$
J_{9, l}=-\int_{-1}^{1} \frac{\tilde{w}_{\varepsilon, l}^{2}}{\bar{H}_{\varepsilon}^{2}}\left(\psi_{\varepsilon}^{\perp}(x)-\psi_{\varepsilon}^{\perp}\left(t_{l}^{\varepsilon}\right)\right) \tilde{w}_{\varepsilon, l}^{\prime} d x=O\left(\varepsilon^{2} D\left(\log \frac{1}{D}\right)^{2}\right)
$$


Now, (B 17) follows from (B 33), (B 34).

Finally, we estimate using (B 29) and $\mu^{\prime}\left(t_{i}\right)=O\left(\sqrt{D} \log \frac{1}{D}\right)$ that

$$
J_{5, l}=\int_{-1}^{1} \mu^{\prime} \phi_{\varepsilon}^{\perp} \tilde{w}_{\varepsilon, l} d x=O\left(\varepsilon \varepsilon \sqrt{D} \log \frac{1}{D} \sqrt{D} \log \frac{1}{D}\right)=O\left(\varepsilon^{2} D\left(\log \frac{1}{D}\right)^{2}\right)
$$

and (B 18) follows. 